\title{
LATE CHALCOLITHIC CERAMIC DEVELOPMENT IN SOUTHERN IRAQI KURDISTAN: THE STRATIGRAPHIC SOUNDING AT KANI SHAIE
}

\author{
By Steve Renette, Khaled Abu Jayyab, Elizabeth Gibbon, Michael P. Lewis, \\ ZANA ABDULLKARIM QADIR, RICARDO CABRAL AND ANDRÉ G. TOMÉ
}

\begin{abstract}
Kani Shaie is a small archaeological site in the Kurdistan Region of Iraq, centrally located in the Bazian Basin, a narrow valley at the western edge of the Zagros Mountains along the major route between Kirkuk and Sulaymaniyah. Its main mound was inhabited almost continuously from the fifth to the middle of the third millennium, c. 5000-2500 B.C.E. This period of Mesopotamian prehistory, corresponding to the Chalcolithic and Early Bronze Age, witnessed major transformations such as initial urbanism and intensification of interregional interaction networks. The recent resurgence of fieldwork in the Kurdistan Region of Iraq is beginning to reveal local trajectories that do not always match the established chronological framework, which is largely based on changes in ceramic technology and styles observed in northern Mesopotamia. Here, we discuss the ceramic sequence retrieved from a step trench at Kani Shaie spanning the entire Late Chalcolithic (c. 4600-3100 B.C.E.). A bottom-up approach to potting traditions at the site allows an initial assessment of the relationship between local communities in the Zagros foothills and large-scale developments in the Mesopotamian world. We argue that the evidence from Kani Shaie reflects a long process in which different communities of practice made active choices of adopting, adapting, or rejecting non-local cultural practices.
\end{abstract}

\section{Introduction}

The Late Chalcolithic (c. 4600-3100 B.C.E.) was a period of major transformations in southwest Asia. From the eastern Mediterranean to Central Asia, societies grew more complex than the village-based societies that came before. Archaeologists have traced the emergence of urban settlements, intricate administrative systems, long-distance trade, and complex political organisation. Consequently, this period features as a major focus of archaeological research aimed at reconstructing the origins of early complex societies in the Middle East (Algaze 1993; Baldi et al. in press; Benati 2018; Butterlin 2003, 2018; Marro 2012; McMahon 2020; Petrie 2013; Postgate 2002; Rothman 2001).

However, our knowledge of Late Chalcolithic (hereafter LC) developments remains uneven. Most of our data come from the Middle and Upper Euphrates region and the Upper Khabur region of Syria and Turkey (Akkermans and Schwartz 2003: 181-210; Frangipane 2018; McMahon 2020; Sagona and Zimansky 2009: 144-171; Schwartz 2001; Ur 2010; Wilkinson and Tucker 1995), and to a lesser extent the eastern Jazira and Tigris region (Abu Jayyab 2012, 2019; Al Quntar and Abu Jayyab 2014; Ball 2003; Butterlin 2009; Gut 1995; Kepinski 2011; Mühl 2013: 101-111; Numoto 1998; Reichel 2008, 2011; Rothman 2002). Decades of research have established that northern Mesopotamia underwent an indigenous development of urbanisation that paralleled the emergence of cities in southern Mesopotamia such as Uruk (Algaze 2008; McMahon 2020; Nissen 2001; Oates et al. 2007; Stein 2012). At the same time, the nature of the relationship between northern and southern complex societies remains unclear. The wide spread during the latter part of the LC of a relatively homogeneous set of material culture (ceramics, administrative practices, and architectural features) that developed first in southern Mesopotamia is usually interpreted as an attempt by southerners to access or control the interregional exchange networks that regulated the flow of raw materials (Algaze 1993). Yet despite much research, archaeologists continue to grapple with issues of chronology and different local responses to cultural encounters that are difficult to reconcile within a single model (most recently, Butterlin 2018: 407-427; Dahl et al. 2013; Porter 2012; Rothman 2013). 
East of the Tigris River, evidence for the LC is much scarcer than in northern Mesopotamia. Our knowledge of this region is almost exclusively reliant on outdated sequences from the first half of the twentieth century. The Kuyunjik Mound at Nineveh is the only site where a complete sequence spanning the entire LC has been documented (Gut 1995). ${ }^{1}$ The lack of welldocumented LC sites in the Trans-Tigridian region, and especially the limited evidence for a southern Mesopotamian presence that is so strikingly visible in northern Mesopotamia, have not gone unnoticed. Only further to the east, at Godin Tepe, is there good evidence for the penetration of southern Mesopotamian, or "Uruk", material culture into the central Zagros (Rothman and Badler 2011). In his landmark study of the Uruk world, Algaze (1993: 63-71) could only speculate about the presence of outposts and colonies between the Tigris River and the Zagros Mountains. More recently, both Rothman (2013) and Matthews (2013), echoing earlier work by Henrickson (1994), have considered that the Diyala River and the road via Kermanshah to Hamadan formed a major route connecting Uruk Mesopotamia with the Iranian highlands. In contrast, Petrie (2014) considers the absence of evidence for Uruk presence in the western Zagros foothills to suggest that the enclave at Godin Tepe was tied to an expansion out of southwest Iran, bypassing the Trans-Tigridian region altogether. ${ }^{2}$ New fieldwork in Iraqi Kurdistan is now rapidly filling in this gap on the archaeological map, resulting in a need for updated chronologies and new interpretive models.

\section{Northeast Iraq (Kurdistan)}

In recent years, the Kurdistan Region of Iraq has become a new hub of archaeological fieldwork. The LC is a primary research focus for several projects targeting sites of this period (Fig. 1) (Carter et al. 2020; Catanzariti et al. 2020; Molist et al. 2019; Nieuwenhuyse et al. 2016; Peyronel and Vacca 2015; Peyronel et al. 2016; Pfälzner et al. 2017; Potts et al. 2019; Saber et al. 2014; Sconzo 2019; Skuldbøl and Colantoni 2016a, 2016b, 2018; Stein 2018; Stein and Alizadeh 2014, 2015; Tsuneki et al. 2015, 2016; Vallet et al. 2017, 2019; Wengrow et al. 2016). While preliminary publications are steadily emerging, as yet there is no complete local ceramic sequence available for the LC. Consequently, projects struggle to relate their data to the frameworks that were established specifically for northern Mesopotamia. Workshops organized in 2018 at the ICAANE in Munich (Baldi et al. in press), at the Freie Universität in Berlin, ${ }^{3}$ and at the fieldwork house of the Kani Shaie Archaeological Project in Bazian exposed the problems with this approach and revealed the need for a better understanding of the ceramic sequence based on locally-derived archaeological datasets. The matter is further complicated by the observation that the region flourished during the first half of the LC (LC1-2), but many sites were subsequently abandoned or drastically reduced in size. The site of Kani Shaie is one of very few documented exceptions, with a continuous sequence of occupation from the Late Ubaid period to the end of the LC and into the Early Bronze Age (EBA). In this article, we present the ceramic corpus from a step trench designed to retrieve a complete stratigraphic sequence of the mound in order to initiate construction of a region-specific ceramic chronology.

\footnotetext{
${ }^{1}$ The early excavations at Tepe Gawra (Rothman 2002; Tobler 1950) have served to identify the early to mid LC in the region. In addition, Iraqi salvage excavations have produced important datasets for the early to mid LC: Qalinj Agha (Hijara 1973); Girdi Resh (Hijara 1976); Tell Begum (Nieuwenhuyse et al. 2016); Greza (Saber et al. 2014); and Tanjero (Saber et al. 2014). For mid to late LC occupation in Iraqi Kurdistan, Abu al-Soof's comprehensive study (1985) was until recently the only source of information.

${ }^{2}$ Evidence from recent fieldwork by Iranian archaeologists and reanalysis of the Mahidasht Survey dataset contradict Rothman's and Matthews' assertions of the presence of
}

centers with "Uruk" pottery, similar to Godin Tepe, in the intermontane plains between Godin Tepe and the Lower Diyala. Earlier reports of such sites were based virtually exclusively on the presence of Beveled Rim Bowls, which on their own cannot be used as a marker of southern Mesopotamian presence (Renette 2018: 315-320; see Renette and Mohammadi Ghasrian 2020 for a synthesis of the present state of knowledge of the Late Chalcolithic in the northern and central Zagros).

3 "Tracing Uruk Pottery Workshop", October 29-30, 2018, organized by R. Bernbeck and S. Pollock as part of the Topoi Excellence Cluster at the Freie Universität, Berlin. 


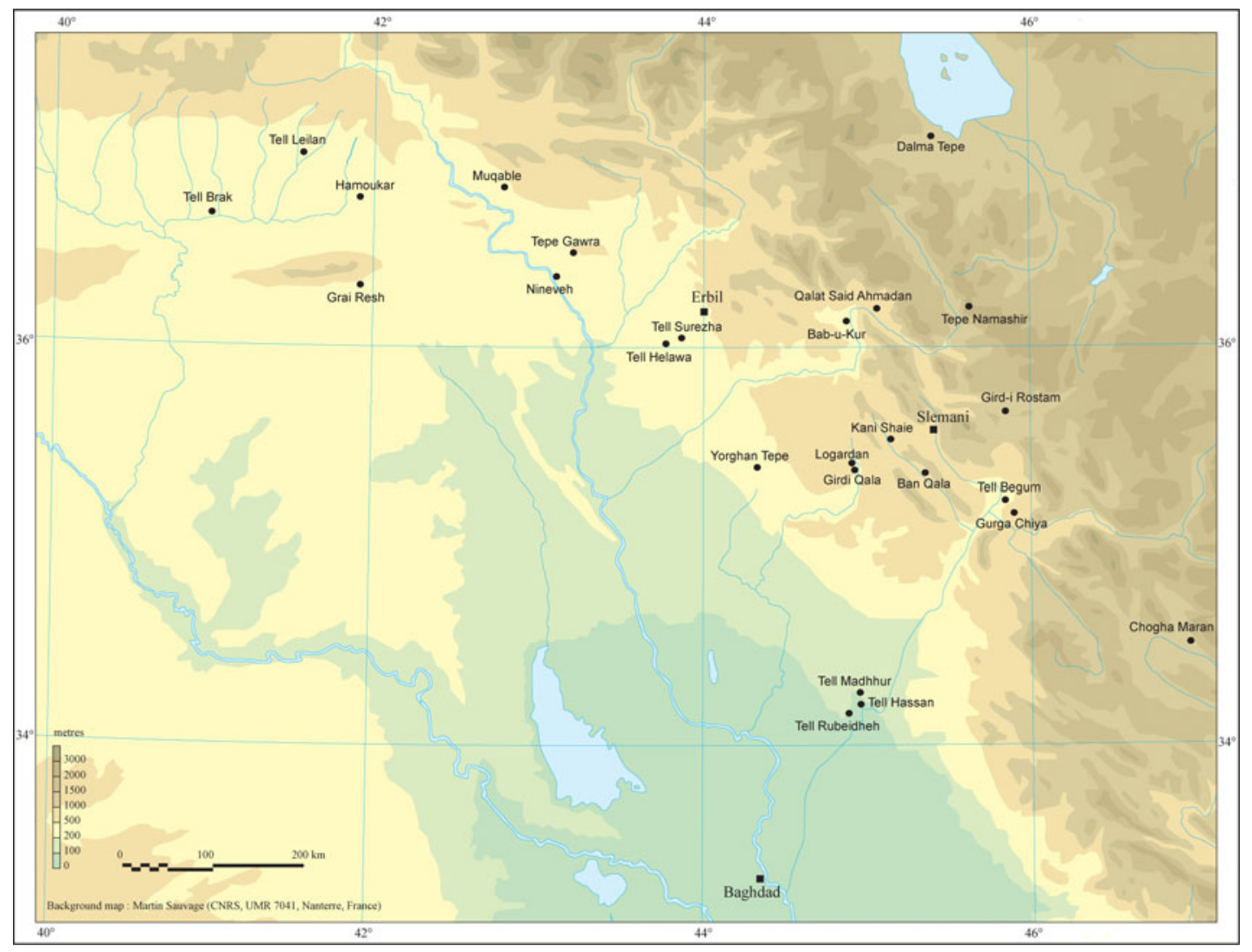

Fig. 1 Map of the northern Zagros Piedmont and the eastern part of northern Mesopotamia with the main Late Chalcolithic sites (base map by M. Sauvage).

\section{Kani Shaie and the Bazian Basin ${ }^{4}$}

Kani Shaie consists of a small mound standing $14 \mathrm{~m}$ above the surrounding surface and covering c. 0.5 ha (Fig. 2) (Renette 2016, 2018: 196-297; Tomé et al. 2016). The site is bounded by the Tainal stream to the west and a spring with a small stream leading into the Tainal along its southern edge. Wrapping around the site to the west, north, and east, a low extension of occupational buildup increases the complete area of the site to c. 3 ha.

Kani Shaie sits at the center of the Bazian Basin, which stretches northwest to southeast for about $35 \mathrm{~km}$, with a width of c. $10 \mathrm{~km}$. This basin separates the hilly Piedmont region around Kirkuk to the west from the Tanjaro-Shahrizor plains and the Zagros Mountains to the east, straddling a stretch of the major road that connects Kirkuk with Sulaymaniyah, along which it forms the first real intermontane valley of the Zagros Mountains.

Between 2013 and 2016, the Kani Shaie Archaeological Project (KSAP) conducted three seasons of excavations at the site. ${ }^{5}$ The primary goals of the project consist of obtaining a stratigraphically anchored sequence of material culture for the Bazian Basin and assessing the position of this region in the development of long-distance interaction between Mesopotamia and the Iranian

\footnotetext{
${ }^{4}$ The Kani Shaie Archaeological Project was initiated in 2012 as a collaboration between S. Renette (University of Pennsylvania), A. Tomé and R. Cabral (University of Coimbra). We would like to thank the Director of the Sulaymaniyah Directorate of Antiquities Kamal Rasheed and the Director of the Sulaymaniyah Museum Hashim Hama for their support and friendship. We are greatly indebted to Zana Abdullkarim, representative of the Directorate of Antiquities,
}

who instantly became an invaluable member of the project. We also extend our gratitude to the entire staff at the Directorate for providing logistical and administrative support, especially S.A. Saber, A. Ameen, and S. Abdulrahman.

${ }^{5}$ KSAP fieldwork has been generously funded by grants from the FCT - Foundation for Science and Technology (Portugal), the Penn Museum, the Louis J. Kolb Foundation, ASOR, and the Explorers Club. 


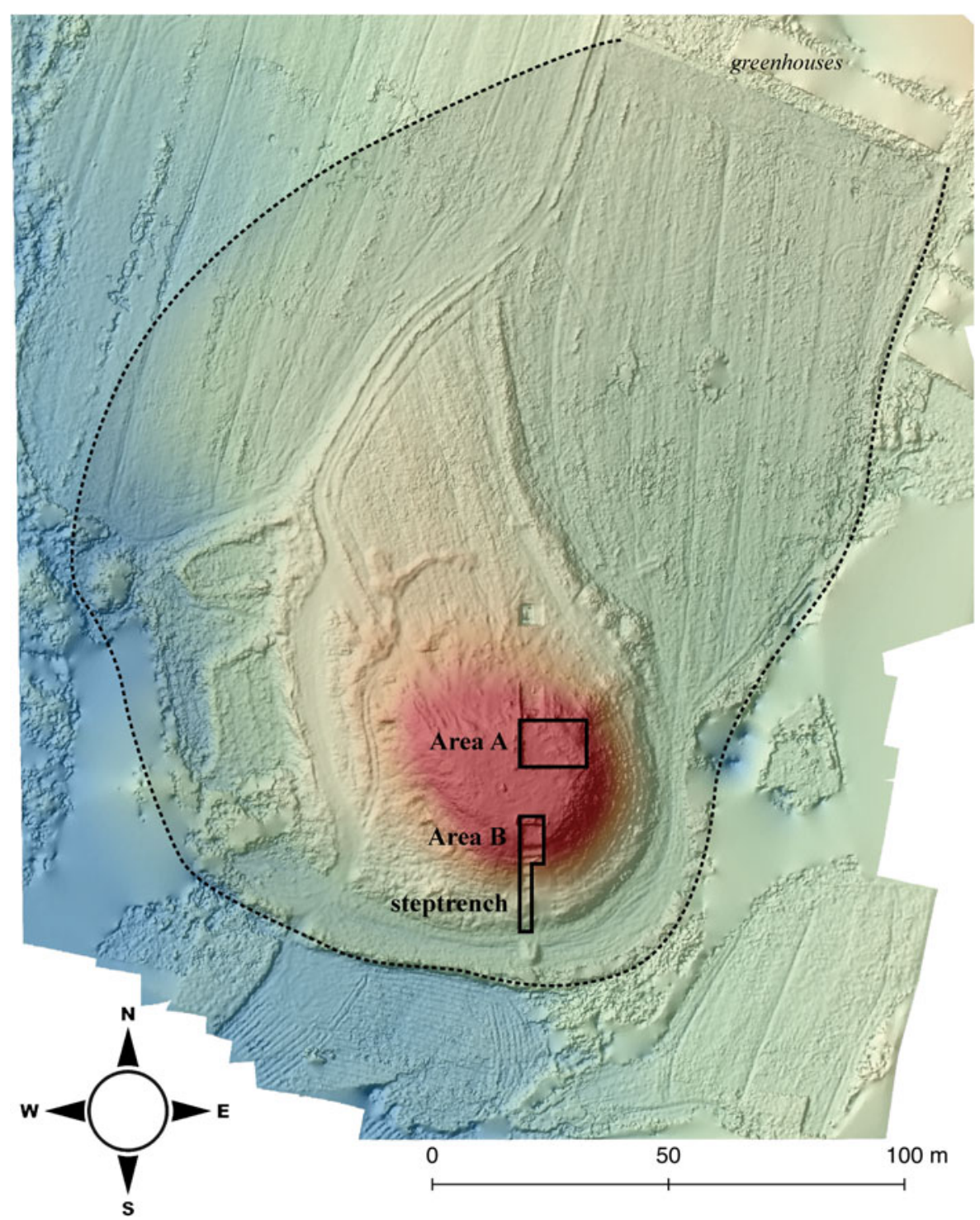

Fig. 2 Digital Elevation Model of Kani Shaie with indication of the main excavation areas.

highlands during the LC and EBA. The site of Kani Shaie was selected because surface collection revealed a long history of occupation that could be used to establish a local ceramic typology before conducting a survey of the basin. ${ }^{6}$ The main mound of Kani Shaie was primarily occupied during the Chalcolithic and EBA, allowing direct access to these occupation levels, while later occupation is mainly spread across the low extension around the mound.

${ }^{6}$ During October-November 2018, KSAP collaborated with J. Giraud and the Mission Archéologique française du Governorate de Soulaimaniah (MAFGS) to conduct an initial survey of the Bazian Basin, funded by the ASOR Mesopotamian Fellowship, French Ministry of Foreign Affairs, Muséum National d'Histoire Naturelle, and CNRS-UMR Team VEPMO. Before KSAP, the Bazian
Basin was visited briefly by E.A. Speiser during his exploration of southern Kurdistan (Speiser 1926-27), and it was included in the Iraq-Jarmo Project survey conducted by R. Braidwood and his team (Braidwood and Howe 1960). As part of this project, B. Howe conducted small-scale excavations at the Epipaleolithic rock shelter of Palegawra (ibid. 28-29, 57-59). 

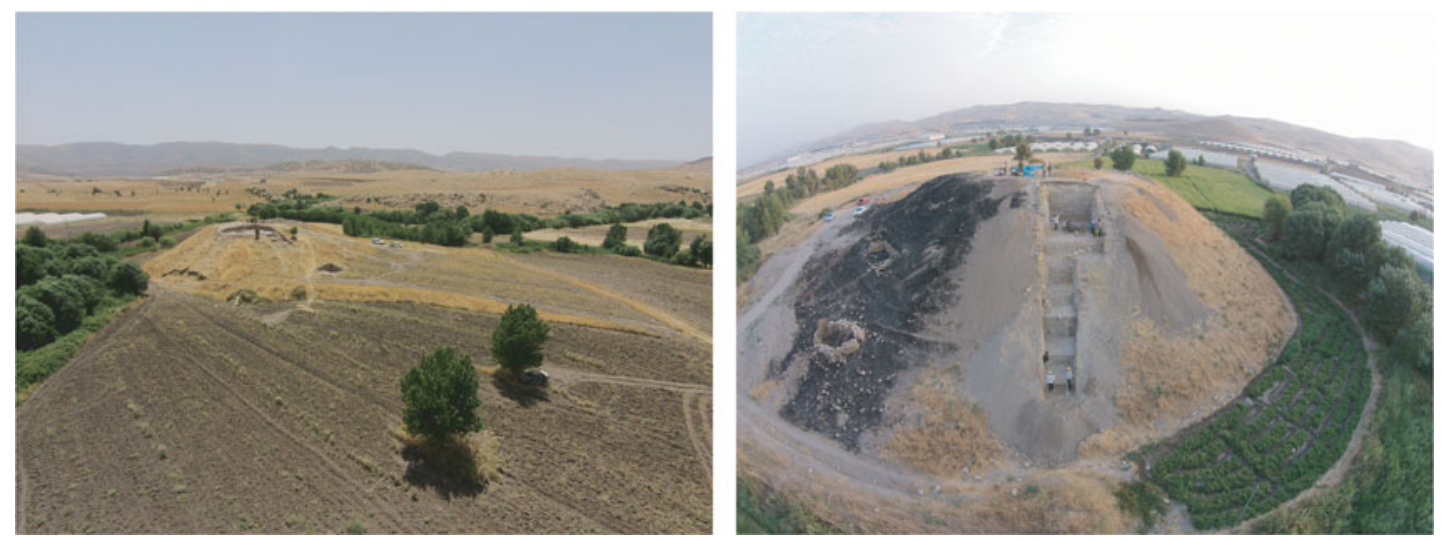

Fig. 3 Drone photos of Kani Shaie and the step trench on the southern slope.

TABle 1 Kani Shaie Main Mound phasing with level descriptions, elevations (measured from permanent point at the site), proposed periodization, and available carbon dates (phase Vd date is from separate pit).

\begin{tabular}{|c|c|c|c|c|c|c|}
\hline \multicolumn{2}{|c|}{$\begin{array}{l}M M \\
\text { phasing }\end{array}$} & levels & $\begin{array}{l}\text { bottom } \\
\text { elevations }\end{array}$ & description & period & $C 14$ \\
\hline \multirow{6}{*}{ V } & \multirow{2}{*}{$\mathrm{a}$} & 1 & 109.75 & poorly preserved surface & LC 4(-5?) & \\
\hline & & 2 & 109.49 & two square spaces defined by mudbrick walls & LC 4(-5?) & \\
\hline & $\mathrm{b}$ & 3 & & hiatus / ephemeral activity zone & LC 4 & \\
\hline & \multirow{2}{*}{$\mathrm{c}$} & 4 & 108.82 & burnt collapse & LC 4 & \multirow[t]{2}{*}{$3530-3370$ cal BC } \\
\hline & & 5 & 108.26 & two square spaces defined by mudbrick walls & LC 3-4 & \\
\hline & $\mathrm{d}$ & 6 & 107.98 & sounding below level 5 & LC 3 & $3770-3665$ cal BC \\
\hline \multirow{6}{*}{ VI } & \multirow{2}{*}{ a } & 7 & 107.02 & poorly preserved occupation level & LC 2(-3) & \multirow{6}{*}{ 4065-3959 cal BC } \\
\hline & & 8 & 106.45 & collapsed mudbrick structure; jar burial & LC 2 & \\
\hline & \multirow{4}{*}{$\mathrm{b}$} & 9 & 106.05 & collapsed fire installation; jar burial & LC 1 & \\
\hline & & 10 & 104.45 & stone wall & LC 1 & \\
\hline & & 11 & 103.52 & two stone-based walls; small fire installation & LC 1 & \\
\hline & & 12 & 102.73 & poorly preserved surface & LC 1 & \\
\hline \multirow{3}{*}{\multicolumn{2}{|c|}{ VII }} & 13 & & pits & late Ubaid & \\
\hline & & 14 & 101.99 & stone paved surface; small fire installation & late Ubaid & \\
\hline & & 15 & 101.15 & stone-based wall & late Ubaid & \\
\hline
\end{tabular}

\section{Kani Shaie phasing}

In 2013, KSAP began a sounding $5 \mathrm{~m}$ wide on the southern slope of the mound (Area B), which was continued down the slope as a $2.5 \mathrm{~m}$ wide step trench in 2015 and 2016 in order to obtain the complete mound sequence within the time constraints (Fig. 3). In addition, a larger area of excavation (Area A) explores the northeastern quadrant of the mound. This open area excavation has so far mainly targeted the EBA occupation, while the LC levels have yet to be reached.

Main Mound phase I (MM I) consists of fragments of modern architectural collapse covering the top of the mound. MM II is a Late Ottoman cemetery from the 18-19th century C.E., while a group of large Middle Islamic pits form MM III. MM IV encompasses the EBA occupation, which is a long sequence of architectural levels that form a deposit c. three to four meters thick.

Here we consider the excavation of the LC levels of the step trench as a separate operation with its own internal sequence of levels, which are grouped as part of the Main Mound phasing (Table 1). This step trench operation exposed at least 15 levels of occupation spanning $8.5 \mathrm{~m}$ of deposits that can be grouped into three separate phases of the main mound (Fig. 4). MM phase V, spanning c. $2.5 \mathrm{~m}$ of deposits, consists of six levels of occupation with relatively large-scale mudbrick architecture. The upper part, phase Va (levels 1-2), is oriented northeast-southwest (Fig. 5a). Level 1 represents the last occupation of the LC, after which there was a hiatus in 


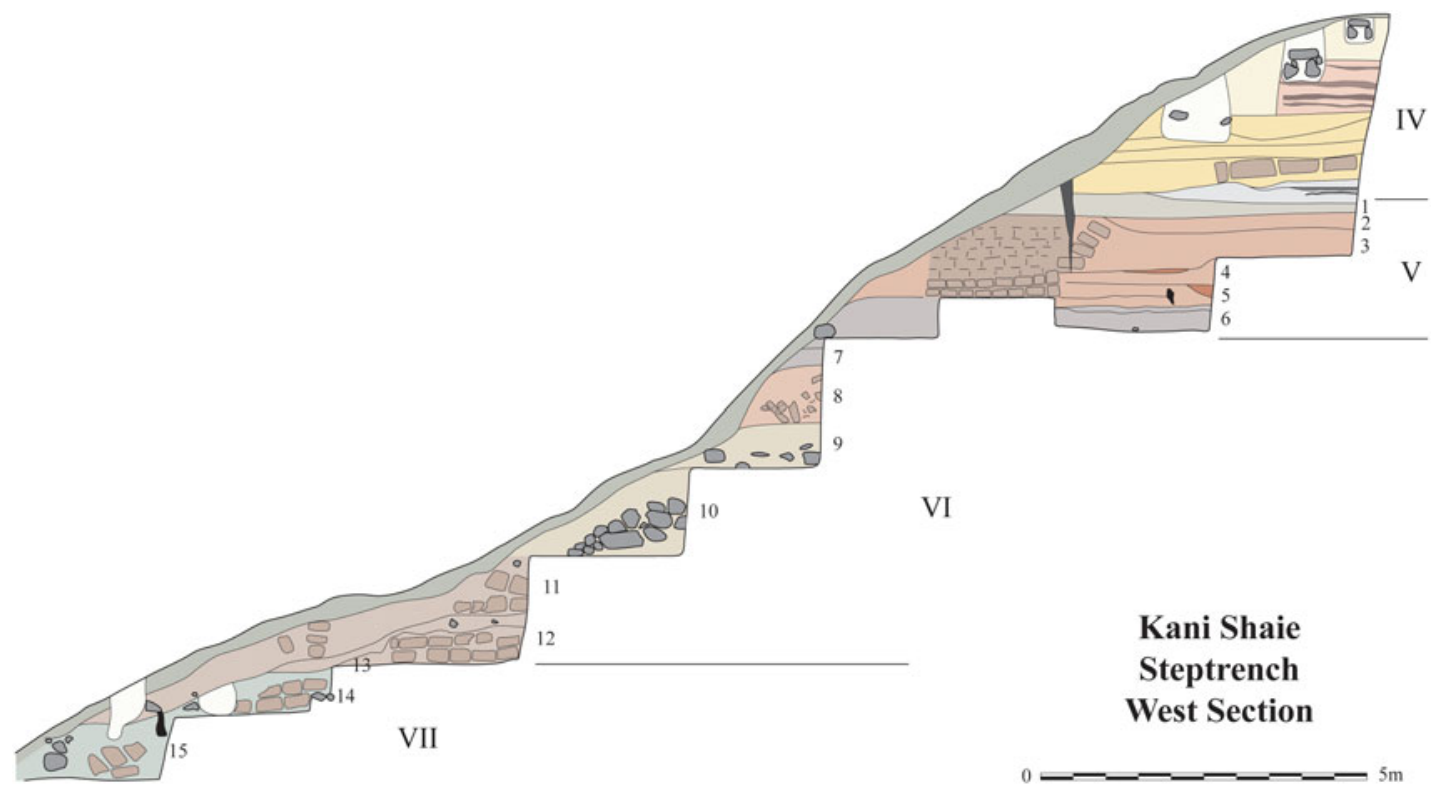

Fig. 4 Section drawing of the west profile of the step trench highlighting the Late Chalcolithic levels.

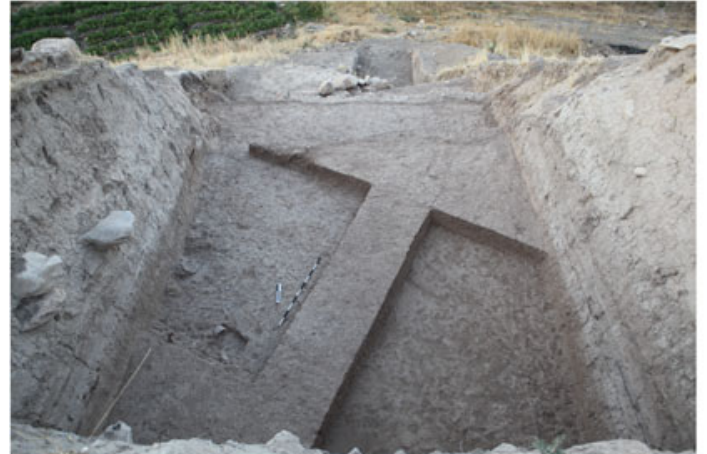

a

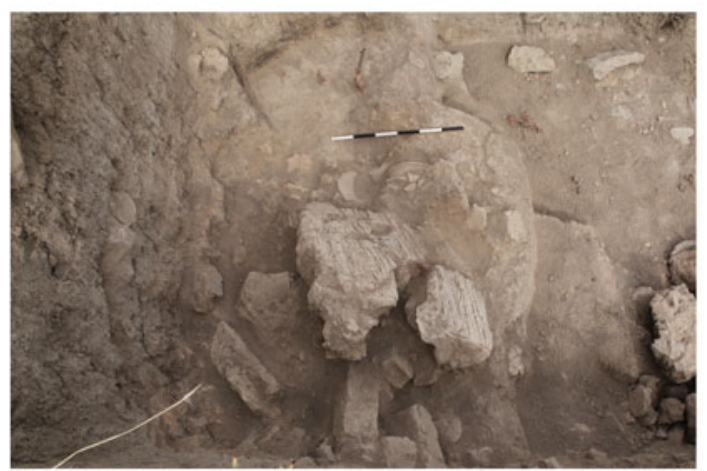

$\mathrm{C}$

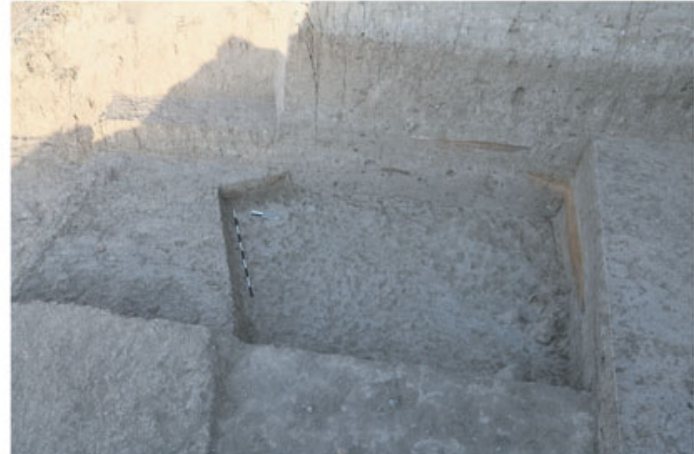

b

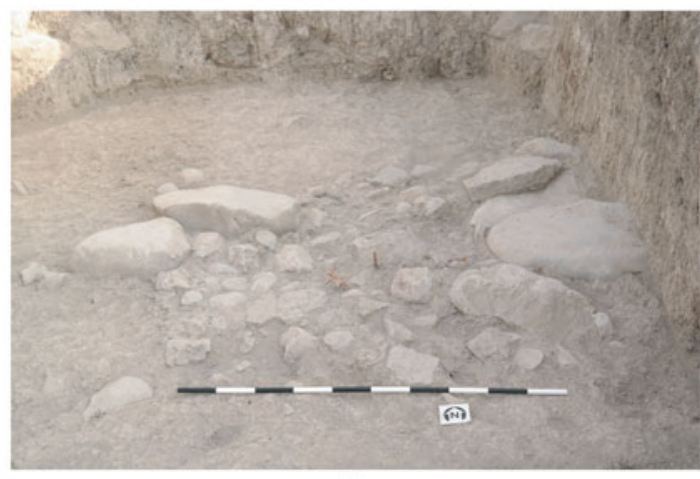

d

Fig. 5 Trench photos: (a) level 2; (b) level 5; (c) level 8; (d) level 14. 
TABLE 2 AMS dates of three LC contexts from Kani Shaie, processed at Beta Analytic (2036-ES.1), DirectAMS (2088-ES.54), and Keck-CCAMS Group Irvine (11002-ES.1).

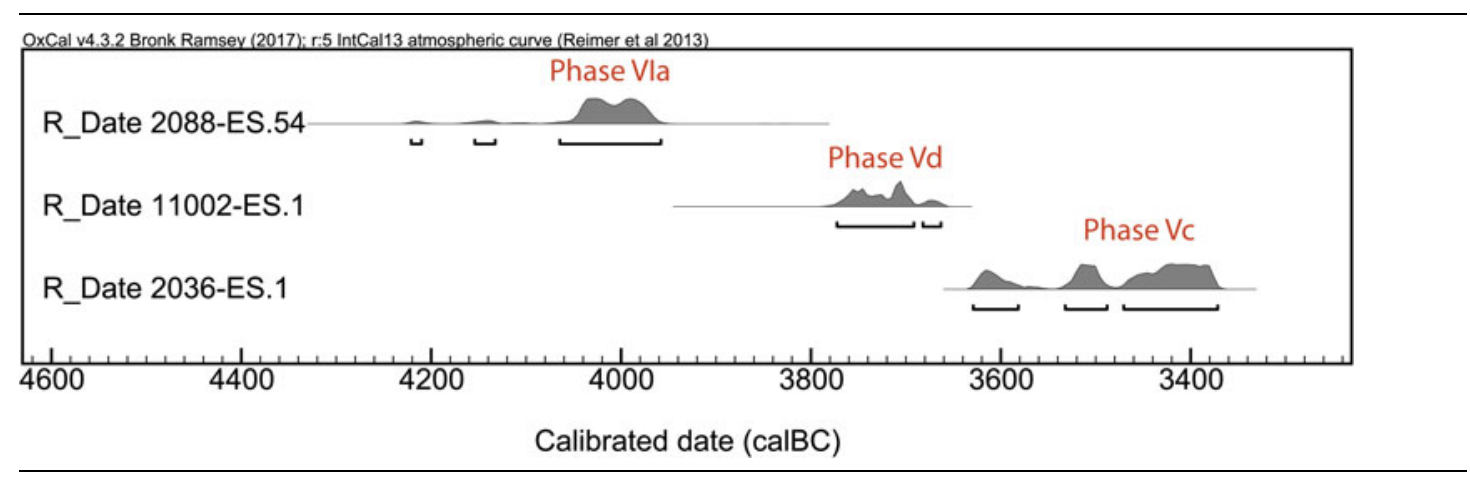

occupation of unknown duration. Extended exposure of this level and intrusive activity of the initial EBA occupation resulted in poor preservation. The subsequent level 2 was much better preserved and sealed by the architecture and surface of level 1 .

Level 3 lacks architectural remains in the step trench sounding. Instead, it is defined by ephemeral activity areas forming subphase $\mathrm{Vb}$, which could represent a brief hiatus in occupation.

Levels 4-5 form subphase Vc, with large-scale mudbrick architecture oriented northwest-southeast. The level 4 building was destroyed by conflagration, as the spaces were filled with burnt collapse. Two C14 dates from level 4 provide a secure dating of 3530-3370 cal B.C.E. (Table 2). Level 5 is an earlier building with a similar layout that was largely cleaned out before the construction of the level 4 building (Fig. 5b). Finally, subphase Vd consists of level 6, which was only reached in a sounding below the floor of one of the level 5 spaces and in a narrow exposure at the edge of the mound in the step trench. A pit lower on the slope that is most likely contemporary with this level, based on ceramic similarities, produced a C14 date between 3770-3665 cal B.C.E. (Table 2).

The next phase, MM VI, which spans ca. $4.5 \mathrm{~m}$ of deposits, is defined by a change in material culture and architectural layout. The upper level 7 was only exposed at the very edge of the mound, where excavations revealed a wall foundation of a single row of stones. Level 8 is particularly well preserved, with the remains of a collapsed mudbrick building (Fig. 5c). Within the pile of burnt bricks and roof fragments were large amounts of pottery, including several broken, but complete, vessels. Underneath the surface of this structure was an intact jar burial carbon dated to 4065-3959 cal B.C.E. (Table 2). This burial contained the remains of a child interred with a cooking pot.

A large collapsed fire installation served to identify the surface of level 9. Level 10 is defined by the presence of a stone wall foundation, but the occupational deposits in this part of the step trench were difficult to identify and were explored at the very end of excavations in 2015 . When excavations resumed in 2016, a year of wind, snow, and rain erosion had further obscured these contexts, necessitating the creation of a new step that consisted of small-scale mudbrick architecture in levels 11-12.

MM phase VII, spanning ca. $1.5 \mathrm{~m}$ of deposits, consists of three levels. The top level 13 is defined by pits that were dug near the edge of the mound without any identifiable corresponding occupation. Levels 14 and 15 contained small, mudbrick architecture on stone foundations, a stone paved surface, and a small fire installation (Fig. 5d). While these remains appear similar to the phase VI settlement layout, the material culture of these levels warrants the identification of a separate phase.

\section{Development of ceramic production in the LC phases at Kani Shaie}

\section{Phase VII}

Phase VII at Kani Shaie encompasses the earliest three occupation levels reached at the site until this point. 448 sherds dating to this phase were recovered, of which 104 were diagnostic (23.2\%). Bowls 
dominate the assemblage at $72.8 \%$, while jars and large basins combine for $27.2 \%$. In total, five bowl and two jar forms dominate the assemblage, representing $70 \%$ of all diagnostic sherds.

Forms

Mass-produced bowls (MPB) ${ }^{7}$ are the most frequent vessel type in phase VII $(18.5 \%){ }^{8}$ This is a coarse straight sided bowl that combines coarse vegetal and mineral temper and was fired at a relatively low temperature (Fig. 6: 1). This vessel type occurs at most sites in northeastern Mesopotamia during LC1-2, at least as far south as the Shahrizor and the Hamrin Basin. In the Kurdistan region of Iraq it is mainly known from sites in the Erbil Plain, including Qalinj Agha level A (Hijara 1973: pl. 23), Surezha (Stein 2018: fig. 8: 3), and Tell Helawa (Peyronel and Vacca 2015: fig. 12: 1-3). Further south, this type is found at Late Ubaid/LC1 levels at Gurga Chiya (Wengrow et al. 2016: fig. 12: 23) and Tell Madhhur (Moon and Roaf 1984: fig. 16: 1-2; Roaf 1989: fig. 3: D3, D11, F16). A similar vessel to the MPB is a wide coarse tray (7\%) (Fig. 6: 2), which seems to be exclusive to the Kurdistan region of Iraq and particularly the Shahrizor, where it has been attested in Late Ubaid/LC1 levels at the sites of Gurga Chiya (Wengrow et al. 2016: fig. 12: 20-22) and Tell Begum (Nieuwenhuyse et al. 2016: fig. 27: 21-22).

Straight-sided bowls with simple rims (11.4\%) occur frequently throughout phase VII (Fig. 6: 5). Half of the examples recovered were finer green/buff wares with dark painted geometric designs. Similar examples were found in Ubaid levels at Qalat Said Ahmadan (Tsuneki et al. 2016: fig. 2.10: 4), Surezha (Stein 2018: fig. 7: 1, 3), and Tell Helawa (Peyronel and Vacca 2015: fig. 10: 3, 7, 10) among many other sites. Variants with an inner ledge (Fig. 6:4) and a grooved rim (Fig. 6: 3), which are always decorated with simple painted bands, are less common at Kani Shaie. Straightsided bowls with an inner ledge occur further north at Qalat Said Ahmadan in Ubaid levels (Tsuneki et al. 2016: fig. 2.10: 13) and Tell Helawa (Peyronel and Vacca 2015: fig. 10: 13). Grooved rim straight-sided bowls have parallels in the Erbil plain at Surezha (Stein 2018: fig. 8: 1-2), Tell Nader (Kopanias et al. 2013: fig. 23: 11), and Tell Helawa (Peyronel and Vacca 2015: fig. 10: 12).

Round bowls with flat rims (Fig. 6: 6) are also relatively common at Kani Shaie (7\%), but they seem to be a locally restricted type with good parallels at nearby Gurga Chiya (Wengrow et al. 2016: fig. 12: 15). Another relatively common vessel (10\%) at Kani Shaie is the in-turned globular bowl (Fig. 6: 11). These bowls have many parallels at sites west of the Tigris River and sites north of the Lower Zab River, but they are not as common south of the Lower Zab.

The most common jar shape (8.4\%) at Kani Shaie is the short neck flaring jar (Fig. 6: 13-14). Similar forms are found at Gurga Chiya during the Late Ubaid/LC1 (Wengrow et al. 2016: fig. 12: 7) and Qalinj Agha level A (Hijara 1973: pl. 23). One example was recovered of a jar with an internal ledge (Fig. 6: 12). This vessel is only present within this phase and is known from most Ubaid sites in the region, such as Surezha (Stein 2018: fig 8: 5), Tell Nader (Kopanias et al. 2013: fig. 23: 14-15), and Tell Helawa (Peyronel and Vacca 2015: fig. 10: 1-2), as well as in the Hamrin at Tell Abada (Jasim 1985: figs. 124-175) and Tell Madhhur (Moon and Roaf 1984: fig. 18: 13). ${ }^{9}$ It is also worth mentioning two jar forms that are found at Kani Shaie at low frequency: grooved rim jars and fine small flaring rim jars (Fig. 6: 8-9) with good parallels at Yorghan Tepe (Starr 1939: pl. 43: E-F). Finally, a single sherd of an extended rim pot was found in this phase (Fig. 6: 10).

A relatively frequent category of vessels from phase VII belongs to the Dalma Impressed ware (Fig. 6: 15-16; Fig. 7: 1-3) (7.7\%). ${ }^{10}$ Dalma Impressed wares have a wide distribution in the

\footnotetext{
${ }^{7}$ MPBs at Kani Shaie are mainly of the "Wide Flower Pot" type (Baldi 2012: type IV, while the earliest MPB sherds might be of his type I). While use of the terminology of Wide Flower Pot and Coba Bowl has varied over the years, authors generally agree that these names encompass a variety of forms that are chronologically sensitive and have different geographical distributions (Baldi 2012; Rothman 2002: 55; Rova 2007: 12). The MPBs of the Zagros Piedmont have not been fully integrated in this debate due to a lack of
}

stratigraphically reliable data. At least for now, the dominance and early occurrence of MPB of the "Wide Flower Pot" type at Kani Shaie seems to confirm Baldi's model (2012).

${ }^{8}$ This is calculated from the percentage of diagnostic rim sherds (n: 70).

${ }^{9}$ The example from Tell Madhhur is an undecorated cooking pot (Moon and Roaf 1984: 147).

${ }^{10}$ Frequency is calculated from the entire sherd collection (n: 104). 

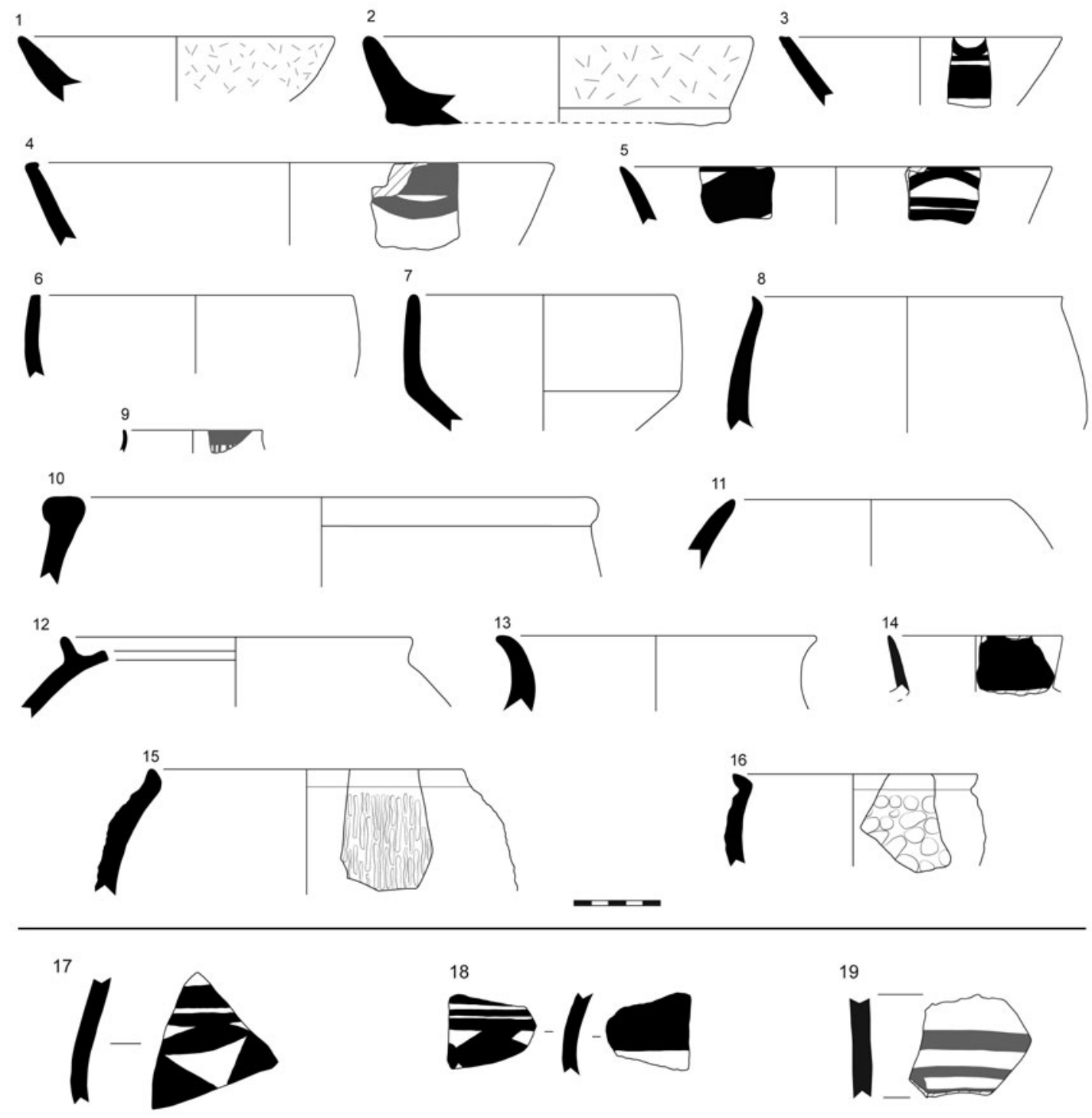

19
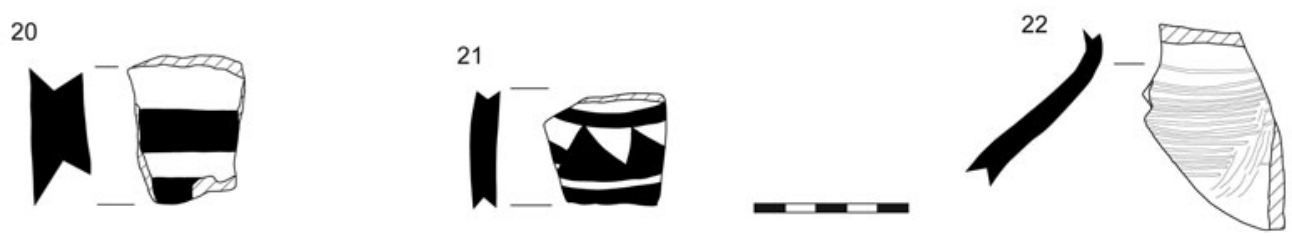

Fig. 6 Phase VII ceramics: 1-14: main vessel types; 15-16: Dalma Impressed ware; 17-21: Ubaid Painted ware; 22: incised ware. See Table 3 for details.

central and northern Zagros, for example at Seh Gabi (Henrickson 1985: fig. 8: 6, 8-11), Chogha Maran, and Tepe Siahbid (Henrickson 1985: fig. 9; Levine and Young 1987: 33), in addition to the type-site Dalma Tepe (Hamlin 1975: pl. IIa, IId, fig. 8: C). Recent work in the Kurdistan region of Iraq has uncovered comparative examples from Gurga Chiya (Wengrow et al. 2016: 265), Surezha (Stein 2018: fig. 11), Uch Tepe 3 (Mühl and Nieuwenhuyse 2016: fig. 15: 7), and as far west as Yorghan Tepe (Starr 1939: pl. 45: E-Q). This distribution shows that the region had ties with the region of Lake Urmia and the central Zagros (Stein 2018: 43). 
TABle 3 Phase VII: description of Fig. 6 illustrated sherds.

\begin{tabular}{|c|c|c|c|c|c|c|c|c|c|}
\hline No. & Type & Fabric & Technology & $\begin{array}{l}\text { Exterior Surface } \\
\text { Treatment }\end{array}$ & $\begin{array}{l}\text { Interior Surface } \\
\text { Treatment }\end{array}$ & $\begin{array}{l}\text { Exterior } \\
\text { Colour }\end{array}$ & $\begin{array}{l}\text { Interior } \\
\text { Colour }\end{array}$ & Core Colour & $\begin{array}{l}\text { Decoration } \\
\text { Colour }\end{array}$ \\
\hline 1 & Wide Flower Pot & F1a & coil & rough & rough & $\begin{array}{l}\text { very pale } \\
\text { brown }\end{array}$ & $\begin{array}{l}\text { very pale } \\
\text { brown }\end{array}$ & very pale brown & \\
\hline 2 & wide coarse tray & F1a & slab / coil & slightly smoothed & slightly smoothed & pale brown & $\begin{array}{l}\text { very pale } \\
\text { brown }\end{array}$ & very pale brown & \\
\hline 3 & $\begin{array}{l}\text { straight-sided bowl with } \\
\text { grooved rim }\end{array}$ & F1c & coil & smoothed & smoothed & pale yellow & pale yellow & pale brown & dark grey \\
\hline 4 & $\begin{array}{l}\text { straight-sided bowl with } \\
\text { inner ledge }\end{array}$ & $\mathrm{F} 1 \mathrm{~b}$ & coil & smoothed & smoothed & $\begin{array}{l}\text { very pale } \\
\text { brown }\end{array}$ & pink & pink & reddish brown \\
\hline 5 & $\begin{array}{l}\text { straight-sided bowl with } \\
\text { simple rim }\end{array}$ & $\mathrm{F} 2 \mathrm{~b}$ & pinched & wet smoothed & wet smoothed & pale brown & pale brown & light grey & dark grey \\
\hline 6 & round bowl with flat rim & F1a & coil & wet smoothed & rough & $\begin{array}{l}\text { very pale } \\
\text { brown }\end{array}$ & pink & grey & \\
\hline 7 & low carinated bowl & $\mathrm{F} 1 \mathrm{~b}$ & mould / coil & slightly smoothed & slightly smoothed & pink & pink & pink & \\
\hline 8 & $\begin{array}{l}\text { globular vessel with } \\
\text { upturned rim }\end{array}$ & $\mathrm{F} 1 \mathrm{~b}$ & coil & rough wipe & rough wipe & $\begin{array}{l}\text { very pale } \\
\text { brown }\end{array}$ & brown & dark grey & \\
\hline 9 & $\begin{array}{l}\text { globular vessel with } \\
\text { upturned rim }\end{array}$ & $\mathrm{F} 4 \mathrm{a}$ & pinched & smoothed & smoothed & pale brown & pale brown & pale brown & $\begin{array}{l}\text { dark reddish } \\
\text { brown }\end{array}$ \\
\hline 10 & extended rim pot & F1a & unknown & wet smoothed & wet smoothed & pink & pink & pinkish grey & \\
\hline 11 & globular bowl & F1a & coil & slightly smoothed & slightly smoothed & $\begin{array}{l}\text { very pale } \\
\text { brown }\end{array}$ & pale brown & light grey & \\
\hline 12 & internal ledge jar & F1b & slab / coil & wet smoothed & rough & $\begin{array}{l}\text { reddish } \\
\text { yellow }\end{array}$ & $\begin{array}{l}\text { reddish } \\
\text { yellow }\end{array}$ & grey & \\
\hline 13 & short neck flaring rim jar & F1b & coil & rough wipe & slightly smoothed & pink & pink & $\begin{array}{l}\text { light brownish } \\
\text { grey }\end{array}$ & \\
\hline 14 & short neck flaring rim jar & F1c & drawn & smoothed & smoothed & light grey & pale yellow & light grey & dark grey \\
\hline 15 & Dalma Impressed jar & F1b & coil & wet smoothed & slip & pinkish white & pink & $\begin{array}{l}\text { light reddish } \\
\text { brown }\end{array}$ & $\begin{array}{l}\text { interior slip: weak } \\
\text { red }\end{array}$ \\
\hline 16 & Dalma Impressed jar & F3a & coil & rough & rough wipe & red & $\begin{array}{l}\text { reddish } \\
\text { yellow }\end{array}$ & grey & \\
\hline 17 & dark painted body sherd & F1c & $\begin{array}{l}\text { coil and wheel } \\
\text { finish }\end{array}$ & self slip & self slip & pale brown & pale brown & pink & dark brown \\
\hline 18 & dark painted body sherd & F1b & $\begin{array}{l}\text { coil and wheel } \\
\text { finish }\end{array}$ & smoothed & smoothed & pale yellow & pale yellow & pale yellow & $\begin{array}{l}\text { dark greyish } \\
\text { brown }\end{array}$ \\
\hline 19 & red painted body sherd & F3a & $\begin{array}{l}\text { coil and wheel } \\
\text { finish }\end{array}$ & wet smoothed & wet smoothed & $\begin{array}{l}\text { very pale } \\
\text { brown }\end{array}$ & light grey & $\begin{array}{l}\text { light brownish } \\
\text { grey }\end{array}$ & $\begin{array}{l}\text { dark reddish } \\
\text { brown }\end{array}$ \\
\hline 20 & dark painted body sherd & $\mathrm{F} 1 \mathrm{~b}$ & coil / drawn & wet smoothed & wet smoothed & pale yellow & pale yellow & pale olive & black \\
\hline 21 & dark painted body sherd & $\mathrm{F} 4 \mathrm{~b}$ & coil & unknown & unknown & pale green & pale green & pale green & black \\
\hline 22 & incised body sherd & $\mathrm{F} 4 \mathrm{~b}$ & coil / drawn & smoothed & smoothed & pale yellow & pale yellow & pale yellow & \\
\hline
\end{tabular}




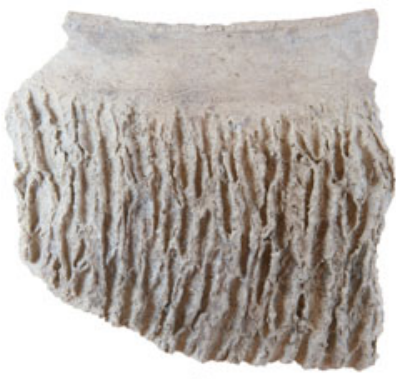

1

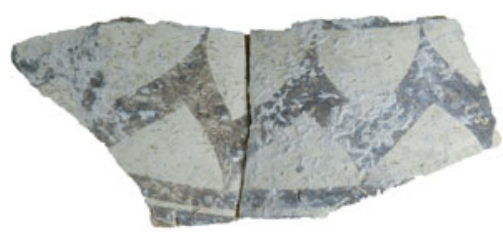

4

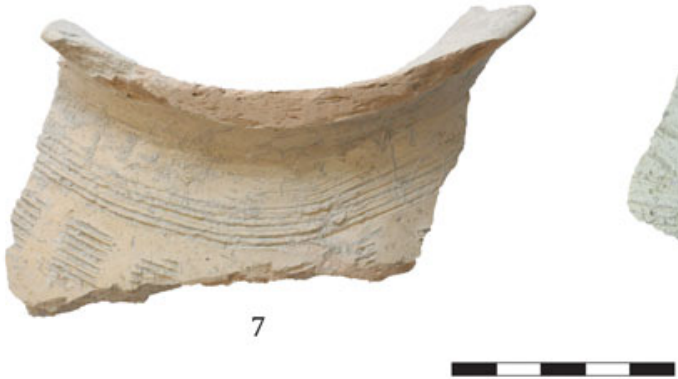

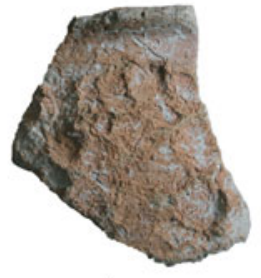

2

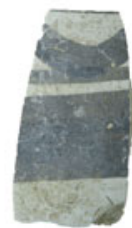

5

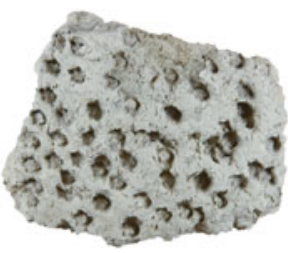

3
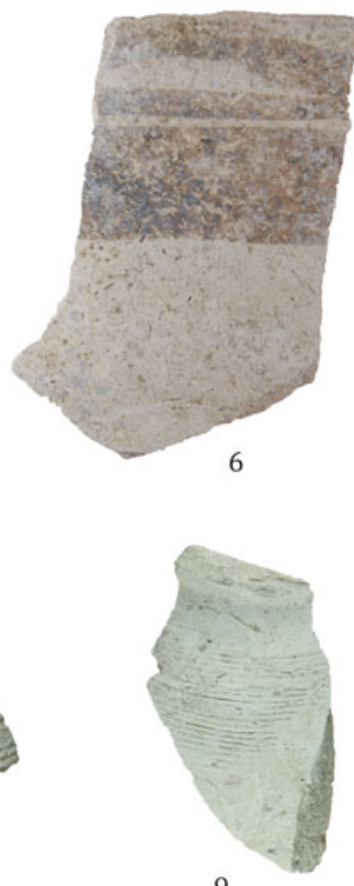

Fig. 7 Phase VII-VIb sherd photos: 1-3: Dalma Impressed ware; 4-6: Ubaid Painted ware; 7-9: LC1 incised ware.

\section{Decoration}

In total, 47 decorated sherds, painted or incised/impressed, were recovered from Phase VII, representing $10.5 \%$ (n: 448) of the total sherds and $45.2 \%$ (n: 104) of diagnostic sherds. Painted decoration is the most common at $8 \%$ of all sherds (n: 36$)$ and $34.6 \%$ of diagnostic sherds (n: 104) and is commonly executed in dark black or brown paint on a buff (n: 22) or light green surface (n: 10) (Fig. 7: 4-6). However, three examples are executed with red paint on a cream or cream slipped surface (Fig. 6: 4, 9, 19), which have parallels at Surezha (Stein and Alizadeh 2017: fig. 14. K). Patterns are limited to a few simple geometric designs. Simple painted bands (Fig. 6: 19-20) and festoons along the rim (Fig. 6: 3-5) are the most common. Other patterns are diagonal bands (Fig. 6: 5), connected lozenges (Fig. 6: 17-18), vertical wavy lines (Fig. 6: 9), and horizontal triangles (Fig. 6: 21). Straight-sided bowls are the most common decorated vessel type (Fig. 6: $3-5$ ), followed by globular bowls and several jars.

Dalma Impressed wares dominate decorated types with surface modification (n: 8). In addition, only three examples of incised decoration were found in phase VII: two comb-incised sherds (Fig. 6: 22) and one with an incised herring-bone pattern. Herring-bone incised vessels are 
commonly found at Late Ubaid sites throughout the region, such as Tanjaro (Saber et al. 2014: fig. 7: 1), Tell Madhhur (Moon and Roaf 1984: fig. 20: 7, 13), Tell Dayim (Takriti 1960: pl. 7: 43), Surezha (Stein and Alizadeh 2014: fig. 12: top), Tepe Gawra level XIII (Tobler 1950: pl. CXXXI: 217), Abu Husaini (Tusa 1984: figs. 33, 36), and Kudish Saghir (Starr 1939: pl. 46: D).

Fabrics

Most of the fabrics seem to be local, ${ }^{11}$ as none of the minerals macroscopically observed seem to have been derived from outside of the immediate vicinity of the site. In total four main fabric groups were observed in the assemblage of phase VII (see Table 4 and Fig. 16, below). The majority of vessels contained vegetal temper to differing degrees, with the exception of $\mathrm{F} 4(5 \%)$ and one example of $\mathrm{F} 3 \mathrm{~b}$.

F1 constitutes $81 \%$ of fabrics (F1a 31\%; F1b 42\%; F1c 8\%). Fabric group F2, which has a finer clay texture than group F1 due to the absence of limestone, represents $9 \%$ of diagnostic sherds. $5 \%$ of diagnostic sherds belong to fabric group F3 (F3a 4\%; F3b 1\%). Finally, another 5\% of diagnostic sherds, all of which are painted and (non-Dalma) incised wares, belong to fabric group F4 (F4a $2 \%$; F4b 3\%). Other painted wares are made from F1b and F1c fabrics, with two examples made from F3. Almost all MPBs and wide coarse trays are made from coarse fabrics F1a, with two examples of MPBs made from F2 heavy chaff tempered fabrics. Dalma Impressed wares are made primarily from fabric F1b, with two examples made from F2 and one example from F3.

Forming Techniques and Surface Treatments

As far as can be observed from fragmentary sherds, coiling or coil building seems to be the predominant technique, comprising $87.5 \%$ of diagnostic sherds. Hand building or pinching accounts for the rest. In many cases coiling is combined with other building or finishing techniques. Moulding the base and applying a coiled body is seen in two examples of bowls with a rounded base and straight vertical walls (Fig. 6: 7). Wheel finishing, most likely on a slow rotation pivot as a means to smoothen and homogenize the surface, is noted on six examples. This seems to be a practice applied in preparation for painting the vessel, however it also seems to be true of one example of a MPB, where the upper half of the body and rim have striation marks. With the exception of two examples, all the hand-drawn vessels observed were finer painted vessels. Handdrawing was usually accompanied by wet smoothing of the vessel surface.

The majority $(67.3 \%)$ of the vessels observed, including decorated wares, ${ }^{12}$ were smoothed by slightly rubbing the surface with a coarse stone or wet smoothed by wiping the surface with a wet cloth or hand. The exceptions to this are the MPBs, coarse trays, and Dalma Impressed wares, as well as individual sherds of larger type groups.

\section{Phase VIb}

Phase VIb produced a total of 343 sherds, 91 of which were diagnostic $(26.5 \%)$. This includes rims (n: 63), bases (n: 2) and decorated body sherds (n: 26). Bowls represent 53.9\% of the assemblage, while jars and large basins combine for $46.1 \%$. In total, eight vessel forms (four bowls and four jars/basins) dominate the assemblage, representing $68 \%$ of all diagnostic rim sherds.

\section{Forms}

The assemblage from phase VIb shares many features with the phase VII assemblage but also diverges in a visible manner (see Fig. 17, below). MPBs continue to be an essential part of the assemblage, comprising $20 \%$ of diagnostic sherds (Fig. 8: 1), while coarse trays remain frequent (6\%) (Fig. 8: 3). ${ }^{13}$ One final vessel form that is still present - albeit at low frequency in both phases VII and VIb $(2.8-3 \%)-$ is a bowl with a rounded base and straight vertical walls, with parallels at Gurga Chiya (Wengrow et al. 2016: fig. 12: 10; fig. 13). Extended rim pots (Fig. 8: 12) increase from the previous level and form $3 \%$ of diagnostic sherds.

\footnotetext{
${ }^{11}$ Fabrics are derived from macroscopic observations conducted on diagnostic sherds.

${ }^{12}$ The exception is Dalma Impressed wares, which had differential treatment between the coarse impressed body
}

and the smoothed rims in the cases where a rim profile was preserved (n: 3).

${ }^{13}$ This is calculated from the percentage of diagnostic rim and base sherds (n: 65). 
TABLE 4 Fabric groups as defined by macroscopic observation during fieldwork and study season.

\begin{tabular}{|c|c|c|c|c|c|c|c|c|c|c|c|c|c|c|}
\hline \multirow{2}{*}{$\begin{array}{l}\text { Fabric } \\
\text { Group }\end{array}$} & \multirow{2}{*}{$\begin{array}{c}\text { Inc. } \\
\text { Density }\end{array}$} & \multicolumn{4}{|c|}{ 1st Inclusions } & \multicolumn{4}{|c|}{ 2nd Inclusions } & \multicolumn{4}{|c|}{ 3rd Inclusions } & \multirow[b]{2}{*}{ comments } \\
\hline & & type & $\%$ & shape & size & type & $\%$ & shape & size & type & $\%$ & shape & size & \\
\hline F1 & \multicolumn{13}{|c|}{ ferruginous clay with natural limestone inclusions and vegetal temper } & \\
\hline 1a & $\geq 15 \%$ & chaff/veg & $10 \%$ & elongated & coarse & limestone & $5 \%$ & sub rounded & $\begin{array}{l}\text { medium- } \\
\text { fine }\end{array}$ & mica & trace & angular & sand & phase V: BRB only \\
\hline $1 \mathrm{~b}$ & $6-10 \%$ & limestone & $5 \%$ & sub rounded & medium & chaff/veg & $2 \%$ & elongated & medium & & & & & \\
\hline $1 \mathrm{c}$ & $\leq 5 \%$ & limestone & $2 \%$ & sub rounded & fine & chaff/veg & $1 \%$ & elongated & fine & & & & & \\
\hline F2 & \multicolumn{14}{|c|}{ ferruginous clay with vegetal temper } \\
\hline $2 \mathrm{a}$ & $\geq 11 \%$ & chaff/veg & $10 \%$ & elongated & coarse & mica & trace & angular & sand & & & & & Chaff-Faced Ware \\
\hline $2 b$ & $6-10 \%$ & chaff/veg & $4 \%$ & elongated & medium & mica & trace & angular & sand & & & & & Chaff-Faced Ware \\
\hline $2 \mathrm{c}$ & $\leq 5 \%$ & chaff/veg & $1 \%$ & elongated & fine & mica & trace & angular & sand & & & & & Chaff-Faced Ware \\
\hline F3 & \multicolumn{14}{|c|}{ micaceous clay } \\
\hline $3 \mathrm{a}$ & $5-8 \%$ & mica & $.5-2 \%$ & sub angular & fine & chaff/veg & $6 \%$ & elongated & medium & & & & & \\
\hline $3 \mathrm{~b}$ & $2-7 \%$ & mica & $.5-2 \%$ & sub angular & fine & limestone & $5 \%$ & sub rounded & medium & & & & & \\
\hline $3 \mathrm{c}$ & $2-7 \%$ & mica & $.5-2 \%$ & sub angular & fine & calcite & $5 \%$ & sub angular & medium & chaff/veg & $2 \%$ & elongated & medium & cooking ware \\
\hline F4 & \multicolumn{14}{|c|}{ mineral-based clay with limestone and ferruginous particles } \\
\hline $4 \mathrm{a}$ & $3-5 \%$ & limestone & $3 \%$ & sub angular & fine & oxides & $1 \%$ & sub angular & sand & & & & & fine limestone \\
\hline $4 \mathrm{~b}$ & $7-10 \%$ & limestone & $7-9 \%$ & sub angular & $\begin{array}{l}\text { medium- } \\
\text { coarse }\end{array}$ & oxides & $1 \%$ & sub angular & sand & & & & & coarse limestone \\
\hline F5 & \multicolumn{14}{|c|}{ calcite tempered cooking ware } \\
\hline $5 \mathrm{a}$ & $1-15 \%$ & calcite & $7 \%$ & (sub) angular & medium & limestone & $5 \%$ & sub angular & medium & chaff/veg & $2 \%$ & elongated & medium & cooking ware \\
\hline $5 b$ & $1-15 \%$ & calcite & $7 \%$ & (sub) angular & medium & limestone & $5 \%$ & sub angular & medium & & & & & cooking ware \\
\hline $5 \mathrm{c}$ & $1-15 \%$ & calcite & $5 \%$ & (sub) angular & medium & chaff/veg & $\leq 5 \%$ & elongated & $\begin{array}{l}\text { medium- } \\
\text { fine }\end{array}$ & mica & trace & angular & sand & cooking ware \\
\hline F6 & \multicolumn{14}{|c|}{ chert tempered clay } \\
\hline 6 & $1-10 \%$ & grey min. & $1 \%$ & angular & fine & chaff/veg & $1 \%$ & elongated & fine & & & & & \\
\hline F7 & \multicolumn{14}{|c|}{ fine clay with natural red mineral inclusions } \\
\hline 7 & $3 \%$ & red min. & $2 \%$ & sub rounded & fine & limestone & $1 \%$ & sub rounded & fine & & & & & \\
\hline F8 & \multicolumn{14}{|c|}{ very fine clay with no visible inclusions } \\
\hline 8 & trace & mica & trace & angular & sand & & & & & & & & & no visible inclusions \\
\hline
\end{tabular}



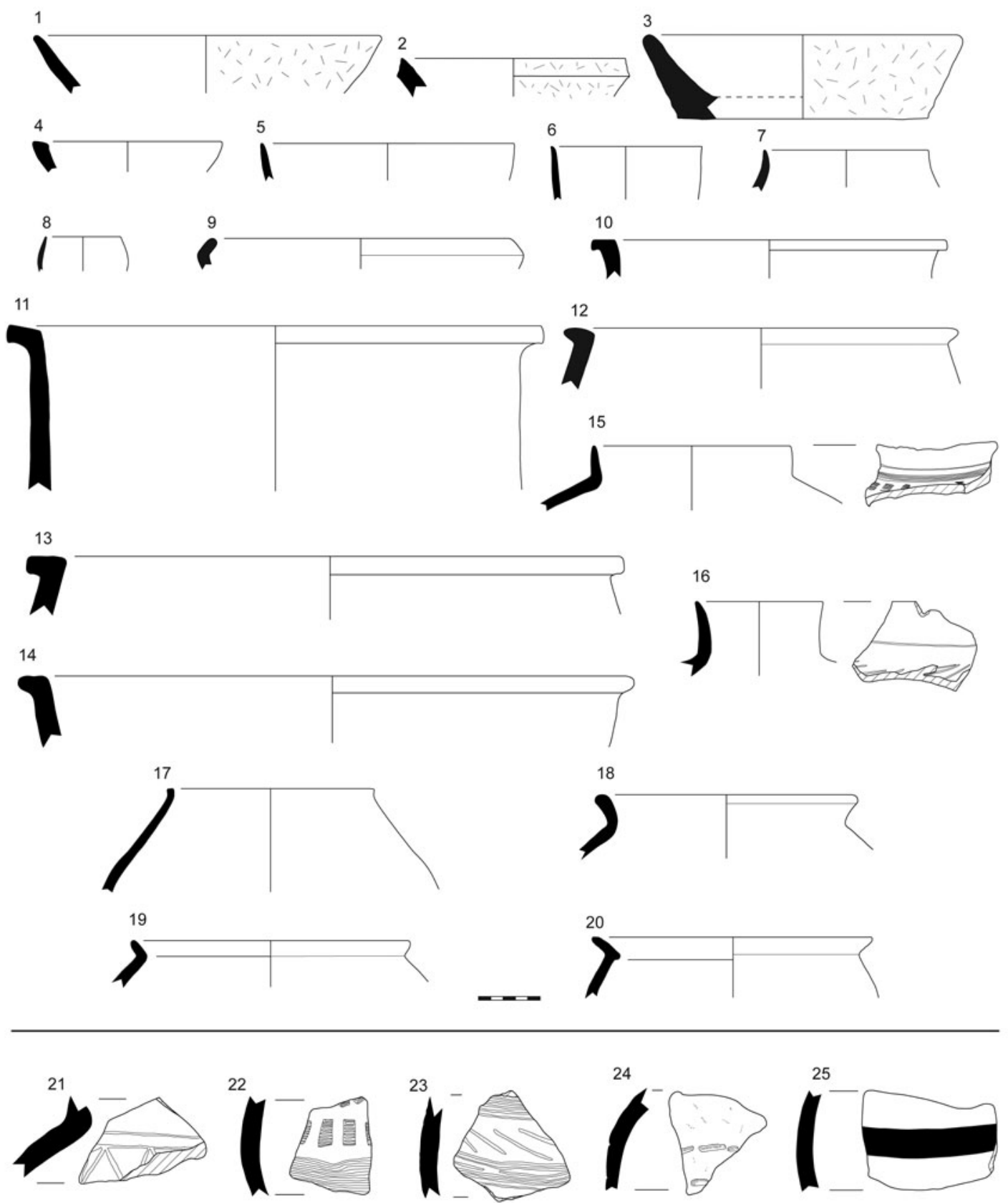

Fig. 8 Phase VIb ceramics: 1-16: main vessel types; 17-20: cooking pots; 21-24: incised ware; 25: painted ware. See Table 5 for details.

There is a significant decrease in a number of features and vessel forms during phase VIb: globular bowls decrease from $10 \%$ to $4.5 \%$, straight-sided bowls from $11.4 \%$ to $3 \%$, straight-sided bowls with inner ledges from $2.8 \%$ to $1.5 \%$, short neck flaring jars from $8.4 \%$ to $1.5 \%$, and to a lesser extent fine small flaring rim jars from $3 \%$ to $1.5 \%$. Most notable is the significant decrease in painted wares. Furthermore, flat rim bowls, straight-sided bowls with grooved rims, jars with internal ledges, and Dalma Impressed wares disappear completely in phase VIb.

Fine ware globular vessels with upturned rims become common in phase VIb (6\%) (Fig. 8: 7). This type is a well-fired thin-walled vessel with fine inclusions, which could have been used as a drinking 
TABle 5 Phase VIb: description of Fig. 8 illustrated sherds.

\begin{tabular}{|c|c|c|c|c|c|c|c|c|c|}
\hline No. & Type & Fabric & Technology & $\begin{array}{l}\text { Exterior Surface } \\
\text { Treatment }\end{array}$ & $\begin{array}{l}\text { Interior Surface } \\
\text { Treatment }\end{array}$ & $\begin{array}{l}\text { Exterior } \\
\text { Colour }\end{array}$ & $\begin{array}{l}\text { Interior } \\
\text { Colour }\end{array}$ & Core Colour & $\begin{array}{l}\text { Decoration } \\
\text { Colour }\end{array}$ \\
\hline 1 & Wide Flower Pot & F1b & coil & wet smoothed & wet smoothed & pale yellow & pale yellow & pale yellow & \\
\hline 2 & Beveled Rim Bowl & F1a & mould & rough & wet smoothed & reddish yellow & $\begin{array}{l}\text { reddish } \\
\text { yellow }\end{array}$ & very dark grey & \\
\hline 3 & wide coarse tray & F1a & $\begin{array}{l}\text { slab / drawn, } \\
\text { coil rim }\end{array}$ & wet smoothed & smoothed & reddish yellow & pale brown & reddish yellow & \\
\hline 4 & $\begin{array}{l}\text { straight sided bowl } \\
\text { with inner ledge }\end{array}$ & F1b & coil & smoothed & smoothed & pink & pink & reddish yellow & \\
\hline 5 & $\begin{array}{l}\text { straight sided bowl } \\
\text { with simple rim }\end{array}$ & F1c & $\begin{array}{l}\text { coil and wheel } \\
\text { finish }\end{array}$ & wet smoothed & wet smoothed & pale brown & pale brown & light grey & \\
\hline 6 & $\begin{array}{l}\text { straight sided cup with } \\
\text { simple rim }\end{array}$ & F1c & $\begin{array}{l}\text { coil and wheel } \\
\text { finish }\end{array}$ & wet smoothed & wet smoothed & $\begin{array}{l}\text { very pale } \\
\text { brown }\end{array}$ & pink & light red & \\
\hline 7 & $\begin{array}{l}\text { globular vessel with } \\
\text { upturned rim }\end{array}$ & F1c & $\begin{array}{l}\text { coil and wheel } \\
\text { finish }\end{array}$ & wet smoothed & wet smoothed & pale brown & pale brown & pale brown & \\
\hline 8 & globular bowl & F1c & coil & smoothed & wet smoothed & reddish yellow & pink & reddish yellow & \\
\hline 9 & high carinated bowl & $\mathrm{F} 5 \mathrm{a}$ & coil & burnished & wet smoothed & pinkish grey & pink & very dark grey & \\
\hline 10 & extended rim pot & F1b & $\begin{array}{l}\text { slab body, coil } \\
\text { rim }\end{array}$ & wet smoothed & rough & pale brown & pink & grey & \\
\hline 11 & extended rim pot & F1a & $\begin{array}{l}\text { slab body, coil } \\
\text { rim }\end{array}$ & smoothed & rough & pink & pink & reddish yellow & \\
\hline 12 & extended rim pot & F1a & $\begin{array}{l}\text { slab body, coil } \\
\text { rim }\end{array}$ & wet smoothed & rough & pale yellow & pale brown & grey & \\
\hline 13 & extended rim pot & F1a & slab & wet smoothed & wet smoothed & $\begin{array}{l}\text { very pale } \\
\text { brown }\end{array}$ & pinkish white & pink & \\
\hline 14 & extended rim pot & F1a & coil & wet smoothed & wet smoothed & pale yellow & pale brown & $\begin{array}{l}\text { light yellowish } \\
\text { brown }\end{array}$ & \\
\hline 15 & slightly flaring rim jar & F1b & $\begin{array}{l}\text { coil and wheel } \\
\text { finish }\end{array}$ & self slip & wet smoothed & $\begin{array}{l}\text { very pale } \\
\text { brown }\end{array}$ & pink & pink & \\
\hline 16 & slightly flaring rim jar & F1a & coil & wet smoothed & wet smoothed & $\begin{array}{l}\text { very pale } \\
\text { brown }\end{array}$ & pale brown & pale yellow & \\
\hline 17 & raised rim cooking pot & $\mathrm{F} 3 \mathrm{a}$ & coil & wet smoothed & rough & $\begin{array}{l}\text { very pale } \\
\text { brown }\end{array}$ & $\begin{array}{l}\text { very pale } \\
\text { brown }\end{array}$ & very pale brown & \\
\hline 18 & $\begin{array}{l}\text { thickened flaring rim } \\
\text { cooking pot }\end{array}$ & $\mathrm{F} 5 \mathrm{a}$ & coil & burnished & burnished & $\begin{array}{l}\text { very pale } \\
\text { brown }\end{array}$ & pale brown & grey & \\
\hline 19 & flaring rim cooking pot & $\mathrm{F} 3 \mathrm{a}$ & coil & burnished & burnished & $\begin{array}{l}\text { light brownish } \\
\text { grey }\end{array}$ & $\begin{array}{l}\text { dark greyish } \\
\text { brown }\end{array}$ & $\begin{array}{l}\text { dark greyish } \\
\text { brown }\end{array}$ & \\
\hline
\end{tabular}


TABLE 5 (Continued)

\begin{tabular}{|c|c|c|c|c|c|c|c|c|c|}
\hline No. & Type & Fabric & Technology & $\begin{array}{l}\text { Exterior Surface } \\
\text { Treatment }\end{array}$ & $\begin{array}{l}\text { Interior Surface } \\
\text { Treatment }\end{array}$ & $\begin{array}{l}\text { Exterior } \\
\text { Colour }\end{array}$ & $\begin{array}{l}\text { Interior } \\
\text { Colour }\end{array}$ & Core Colour & $\begin{array}{l}\text { Decoration } \\
\text { Colour }\end{array}$ \\
\hline 20 & flaring rim cooking pot & F3a & coil & burnished & burnished & $\begin{array}{l}\text { light brownish } \\
\text { grey }\end{array}$ & $\begin{array}{l}\text { greyish } \\
\text { brown }\end{array}$ & dark grey & \\
\hline 21 & incised body sherd & F1c & coil & wet smoothed & rough & pale yellow & $\begin{array}{l}\text { very pale } \\
\text { brown }\end{array}$ & $\begin{array}{l}\text { light yellowish } \\
\text { brown }\end{array}$ & \\
\hline 22 & incised body sherd & $\mathrm{F} 2 \mathrm{a}$ & coil & wet smoothed & rough & pink & pinkish grey & reddish yellow & \\
\hline 23 & incised body sherd & F1a & coil / drawn & smoothed & rough & pale yellow & pale brown & pale brown & \\
\hline 24 & impressed body sherd & F1b & coil & wet smoothed & rough & $\begin{array}{l}\text { light greenish } \\
\text { grey }\end{array}$ & light grey & light grey & \\
\hline 25 & $\begin{array}{l}\text { dark painted body } \\
\text { sherd }\end{array}$ & F1c & coil & self slip & scraped & pale brown & pale brown & pale brown & black \\
\hline
\end{tabular}


vessel, with parallels at Tell Helawa in the Erbil plain (Peyronel and Vacca 2015: fig. 12: 17-18). Several new types appear in phase VIb. Wide deep basins with vertical or slightly angled bodies become common $(7.5 \%)$, albeit in a variety of forms. They all have large diameters and outwardly extended ledge rims with variation in shapes (Fig. 8: 10-11, 13-14). Parallels are found across the Kurdistan region of Iraq and beyond at Gurga Chiya (Wengrow et al. 2016: fig. 12: 17-19), Surezha (Stein and Alizadeh 2017: fig. 14: A), Tepe Gawra level XIIA (Tobler 1950: pl. CXXXVI: 274, 277), Gird-i Pasha (Mühl and Nieuwenhuyse 2016: fig. 16: 2-4), Tell Begum (Nieuwenhuyse et al. 2016: fig. 26: 10), Tell Madhhur (Roaf 1989: fig. 5: H7, K7), and Yorghan Tepe (Starr 1939: pl. 49: C). Two vessels of this type were used as child burial urns at Kani Shaie (one in phase VIb and one in phase VIa).

Slightly flaring rim jars continue (6\%) but frequently have incised decoration (Fig. 8: 15-16). One of the more common new types found in VIb is a grey, slightly burnished, flaring rim cooking pot (15.2\%) (Fig. 8: 18-20). This type is primarily calcite tempered, which distinguishes it from other vessels in the assemblage. Parallels were found at Gurga Chiya in Ubaid/LC1 levels (Wengrow et al. 2016: fig. 12: 4-6). This type is the first formal cooking pot in the Kani Shaie sequence and continues throughout the LC. Finally, phase VIb also produced the first example of a Beveled Rim Bowl (BRB) (Fig. 8: 2).

Decoration

During phase $\mathrm{VIb}$, decoration drops from $10.5 \%$ to $7.5 \%$ (n: $26 ; 28.5 \%$ of diagnostic sherds). In a reversion to phase VII, incised wares occur more frequently than painted wares. In total only four painted sherds with simple bands (Fig. 8: 25) (1.1\% of sherds; $4.4 \%$ of diagnostics) were recovered, compared to 22 incised sherds ( $6.4 \%$ of sherds; $24.1 \%$ of diagnostics).

The most common decoration type consists of fine (comb-)incised lines both straight and wavy, primarily executed on jars (Fig. 7: 7-9; Fig. 8: 15-16, 21-23), with parallels at Tell Madhhur (Roaf 1989: fig. 8: J7, J11; fig. 9: J6, J11; fig. 10: K6), Gurga Chiya (Wengrow et al. 2016: fig. 12: 1-2), Surezha (Stein and Alizadeh 2014: fig. 12: top; Stein and Alizadeh 2017: fig. 14: C), and Yorghan Tepe (Starr 1939: pl. 49: C). Occasionally, this decoration is accompanied by herring-bone pattern incisions or stop-gap lines (Fig. 8: 15, 22) as at Tell Madhhur (Roaf 1989: fig. 8: F3, H6, H11; fig. 9: K11; fig. 10: K6) and Surezha (Stein and Alizadeh 2014: fig. 12: top; Stein and Alizadeh 2017: fig. 14: C). Other incised patterns are executed as zigzags or curved slashes. Two examples of impressed applique rope pattern were also recovered from this phase.

Fabrics

F1 continues to dominate the assemblage at 77\% (F1a 28\%; F1b 27\%; F1c 22\%) (Fig. 16). F2 drops slightly from $9 \%$ to $4 \%$ in phase VIb, while $\mathrm{F} 3$ increases from $5 \%$ to $9 \%$. F4 drops from $5 \%$ to $2 \%$, coinciding with the decline in painted wares. A new fabric group, F5 (together with F3c), appears during phase VIb, making up $8 \%$ of recorded fabrics, primarily restricted to burnished grey cooking pots. Of these, only one vessel belonged to subgroup F5b without vegetal additions.

Forming Techniques and Surface Treatments

Coiling remains the most common forming technique, at $96.5 \%$ of the assemblage. Coiling is combined with other construction and finishing techniques, such as pinching and slow wheel finishing. Only rare examples were completely produced by moulding and pinching. The majority of sherds were smoothed or wet smoothed $(77.5 \%)$. Only $7.8 \%$ were left with rough surfaces, primarily MPBs. Burnishing was present on $13 \%$ of vessels, primarily reserved for grey cooking pots.

\section{Phase VIa}

Phase VIa produced 526 sherds, of which 112 were diagnostic $(21.3 \%)$, with bowls being the most common form type (56\%), followed by jars (37\%) and basins (7\%). The most distinct difference between the phase VIa assemblage and that of earlier levels is the complete absence of any decoration.

Forms

Several forms disappear completely by phase VIa: carinated bowls, straight-sided bowls with inner ledges, and coarse trays. Other forms are on their way out during phase VIa: MPBs comprise only 


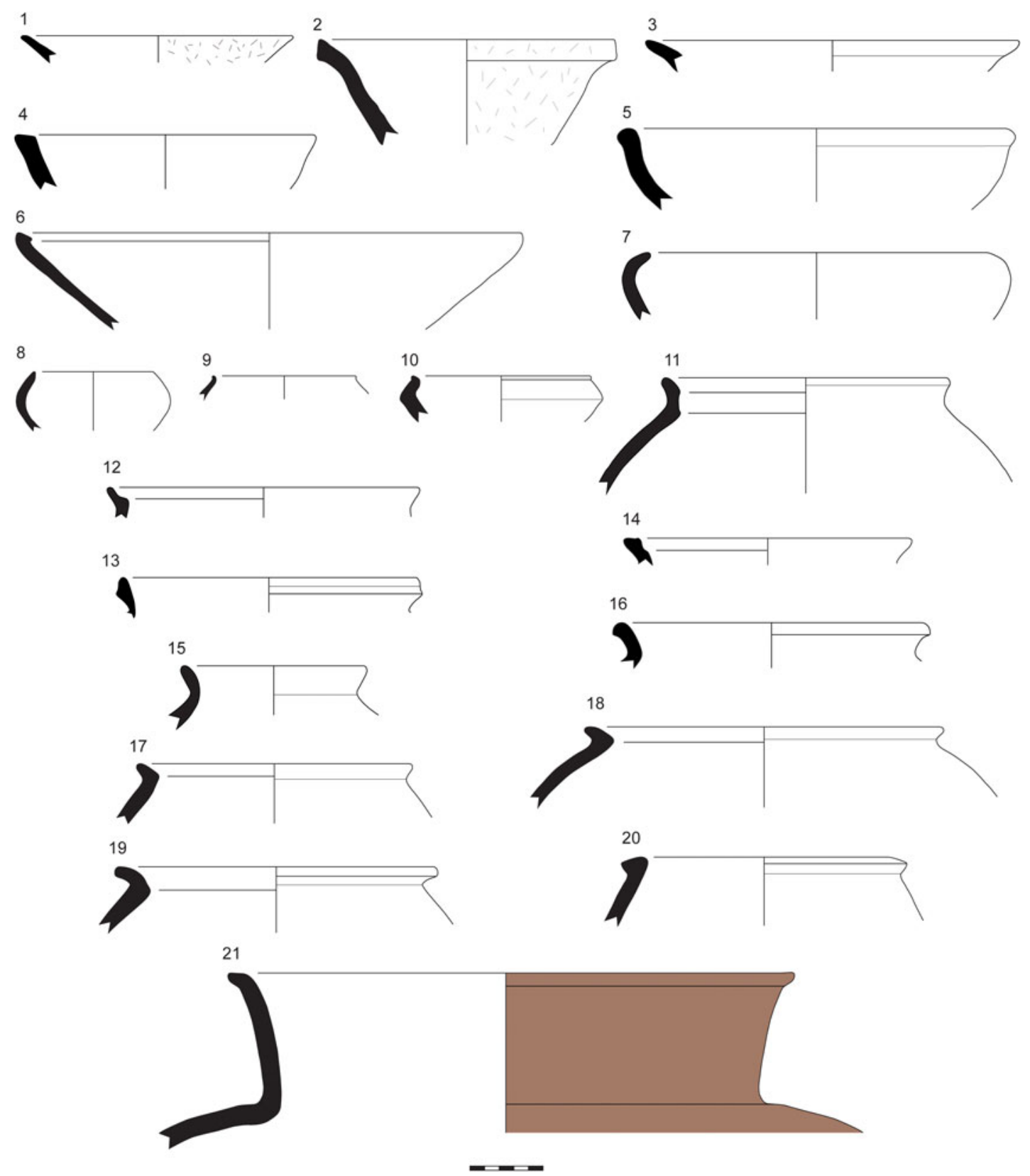

Fig. 9 Phase VIa ceramics: 1-14 and 21: main vessel types; 15-20: cooking pots. See Table 6 for details.

$9 \%$ of the assemblage, compared to $20 \%$ in phase VIb (Fig. 9: 1); in-turned globular bowls decrease from $10 \%$ of the assemblage in phase VII to just $2.7 \%$ (Fig. 9: 8 ); straight-sided bowls with simple rims decrease from $11.4 \%$ in phase VII to only $0.9 \%$.

Other types continue at relatively stable or similar frequencies when compared to the preceding phase VIb. These include fine ware drinking cups with upturned rims (5.5\%) (Fig. 9: 9), grey burnished cooking pots (16.9\%) (Fig. 9: 15-20), collared flaring rim jars (3.5\%) (Fig. 9: 13), and deep basins $(7 \%)$. Just a couple of types increase in frequency from preceding levels, including Beveled Rim Bowls (BRB) (3.5\%) (Fig. 9: 2) and flat rim bowls (3.6\%) (Fig. 9: 4).

Many new forms appear during phase VIa. The majority of these new forms are various types of bowls, such as flaring rim bowls (1.8\%) (Fig. 9: 3), bowls with internal lips (1.8\%), slightly outturned 
TABle 6 Phase VIa: description of Fig. 9 illustrated sherds.

\begin{tabular}{|c|c|c|c|c|c|c|c|c|c|}
\hline No. & Type & Fabric & Technology & $\begin{array}{l}\text { Exterior Surface } \\
\text { Treatment }\end{array}$ & $\begin{array}{l}\text { Interior Surface } \\
\text { Treatment }\end{array}$ & Exterior Colour & $\begin{array}{l}\text { Interior } \\
\text { Colour }\end{array}$ & Core Colour & Slip Colour \\
\hline 1 & Wide Flower Pot & F1a & coil & slightly smoothed & slightly smoothed & pink & $\begin{array}{l}\text { very pale } \\
\text { brown }\end{array}$ & grey & \\
\hline 2 & Beveled Rim Bowl & F1a & mould & rough & rough & reddish yellow & pink & dark grey & \\
\hline 3 & flaring rim bowl & F1b & coil & wet smoothed & wet smoothed & $\begin{array}{l}\text { very pale } \\
\text { brown }\end{array}$ & $\begin{array}{l}\text { very pale } \\
\text { brown }\end{array}$ & $\begin{array}{l}\text { very pale } \\
\text { brown }\end{array}$ & \\
\hline 4 & flat rim bowl & F1b & coil & wet smoothed & wet smoothed & pink & pink & light grey & \\
\hline 5 & slightly out-turned bowl & $\mathrm{F} 3 \mathrm{~b}$ & $\begin{array}{l}\text { coil and wheel } \\
\text { finish }\end{array}$ & burnished & burnished & pink & pink & pink & \\
\hline 6 & $\begin{array}{l}\text { internally beveled rim } \\
\text { bowl }\end{array}$ & F1a & coil & slightly smoothed & slightly smoothed & $\begin{array}{l}\text { very pale } \\
\text { brown }\end{array}$ & $\begin{array}{l}\text { very pale } \\
\text { brown }\end{array}$ & $\begin{array}{l}\text { reddish } \\
\text { yellow }\end{array}$ & \\
\hline 7 & squat inturned bowl & $\mathrm{F} 2 \mathrm{~b}$ & coil & smoothed & wet smoothed & pinkish grey & pink & grey & \\
\hline 8 & globular bowl & $\mathrm{F} 2 \mathrm{~b}$ & mould / coil & smoothed & slightly smoothed & pale brown & pale brown & pink & \\
\hline 9 & $\begin{array}{l}\text { globular vessel with } \\
\text { upturned rim }\end{array}$ & $\mathrm{F} 4 \mathrm{a}$ & coil & smoothed & smoothed & $\begin{array}{l}\text { very pale } \\
\text { brown }\end{array}$ & $\begin{array}{l}\text { pinkish } \\
\text { white }\end{array}$ & pinkish white & \\
\hline 10 & $\begin{array}{l}\text { carinated bowl with } \\
\text { upturned rim }\end{array}$ & F5a & mould / coil & burnished & burnished & dark grey & grey & dark grey & \\
\hline 11 & internally hollowed jar & F1a & coil & slip & wet smoothed & pale brown & $\begin{array}{l}\text { very pale } \\
\text { brown }\end{array}$ & $\begin{array}{l}\text { very pale } \\
\text { brown }\end{array}$ & $\begin{array}{l}\text { very dark } \\
\text { grey }\end{array}$ \\
\hline 12 & grooved rim basin & F1b & coil & slip & smoothed & pink & pink & reddish grey & light red \\
\hline 13 & flaring rim jar & $\mathrm{F} 3 \mathrm{~b}$ & coil & smoothed & smoothed & pinkish grey & light brown & grey & \\
\hline 14 & internally hollowed jar & F1b & coil & slightly smoothed & slightly smoothed & grey & grey & grey & \\
\hline 15 & $\begin{array}{l}\text { restricted flaring rim } \\
\text { cooking pot }\end{array}$ & F5a & coil & smoothed & rough wipe & dark grey & grey & dark grey & \\
\hline 16 & $\begin{array}{l}\text { thickened flaring rim } \\
\text { cooking pot }\end{array}$ & F5a & coil & smoothed & slightly smoothed & dark grey & dark grey & $\begin{array}{l}\text { dark reddish } \\
\text { grey }\end{array}$ & \\
\hline 17 & flaring rim cooking pot & $\mathrm{F} 5 \mathrm{a}$ & coil & burnished & rough wipe & $\begin{array}{l}\text { light brownish } \\
\text { grey }\end{array}$ & grey & dark grey & \\
\hline 18 & flaring rim cooking pot & $\mathrm{F} 5 \mathrm{a}$ & coil & smoothed & slightly smoothed & light brown & light brown & dark grey & \\
\hline 19 & flaring rim cooking pot & $\mathrm{F} 3 \mathrm{c}$ & coil & burnished & rough & $\begin{array}{l}\text { light reddish } \\
\text { brown }\end{array}$ & pink & dark grey & \\
\hline 20 & $\begin{array}{l}\text { extended rim cooking } \\
\text { pot }\end{array}$ & F5b & coil & burnished & rough & $\begin{array}{l}\text { light brownish } \\
\text { grey }\end{array}$ & pink & grey & \\
\hline 21 & flared jar with flat rim & F1a & coil & slip and smoothed & rough wipe & reddish yellow & pink & grey & red \\
\hline
\end{tabular}


bowls (3.6\%) (Fig. 9: 5), and squat in-turned bowls (12.5\%) (Fig. 9: 7). Internally beveled rim bowls become common (9.8\%) (Fig. 9: 6), with parallels throughout northeastern Mesopotamia, including Tell Brak (Oates 2012: fig. 7: 5) and Hamoukar (Abu Jayyab 2012: fig. 8: 12-13). One example of a small, black burnished carinated bowl (Fig. 9: 10) also has parallels at Tell Brak (Abu Jayyab 2012: fig. 15) and at Qalinj Agha and Helawa (Peyronel and Vacca 2015: fig. 13: 12). Other new forms include one example of a grooved rim basin (Fig. 9: 12), similar to a type found at nearby Logardan (Vallet et al. 2017: fig. 18: 7), and internally hollowed jars (2.7\%) (Fig. 9: 11, 14) that are well-attested with a wide distribution in LC2, such as at Tell Helawa (Peyronel and Vacca 2015: 13. 4, 6), Nineveh (Gut 1995: fig. 55), Tell Brak (Oates 2012: fig. 7: 1), and Hamoukar (Abu Jayyab 2012: fig. 9: 4).

Fabrics

F1 still dominates the assemblage in phase VIa, albeit with a significant decrease to $50 \%$ (F1a 24\%; F1b 22\%; F1c 4\%), while F2 increases significantly from 4\% to $22 \%$ in phase VIa (Fig. 16). F3 drops slightly from $9 \%$ to $6 \%$, and F4 only comprises $2 \%$ of the assemblage, consistent with the continuing decline of painted wares. Finally, F5 increases in frequency from $8 \%$ to $20 \%$, reflecting the increased presence of cooking wares.

Forming Techniques and Surface Treatments

Coiling remains the most common forming technique and has been identified in $95.5 \%$ of the assemblage. Coiling is sometimes combined with other techniques, such as a moulded base with coiled upper body. This combination is primarily identified on squat in-turned bowls (Fig. 9: 7). Moulding of the entire vessel is largely restricted to BRBs. Two sherds have traces of a combination of coil construction and wheel finishing: a globular vessel with upturned rim and a slightly outturned bowl.

Regarding surface treatment, the majority of vessels are slightly smoothed $(70 \%)$ by rubbing the surface with a coarse stone or wet smoothed by wiping the surface with a wet cloth or hand. Some vessels, primarily the MPBs, are left without any treatment (17\%). Burnishing occurs in $10 \%$ of the assemblage and is primarily applied on cooking pots, with the exception of one black burnished carinated bowl. The use of red slip and smoothing first makes its appearance during phase VIa and is applied on $2.6 \%$ of the assemblage (Fig. 9: 21).

\section{Phase Vd}

314 sherds were recovered from Phase $\mathrm{Vd}$, of which 83 were diagnostic ( $26.4 \%$ of the total sherd count), including 75 rim sherds (23.9\% of the total sherd count). The remaining diagnostic sherds are bases and lugs. 58 bowl fragments amount to $77.3 \%$ of the rim sherd count, while 17 jar rim sherds form $22.7 \%$.

\section{Forms}

The dominant form within Phase Vd are BRBs (57.3\% of the rim sherd count) ranging from a typical oblique profile and beveled rim to a sharply flaring rim (Fig. 10:9-11). Most are very pale brown in colour with an oxidised core. Other examples range from reddish yellow through to light grey in colour and feature reduced cores.

Much finer, thin-walled bowls with a beveled rim are a chronologically sensitive form confined to phase $\mathrm{Vd}$ and early phase $\mathrm{Vc}$, albeit relatively rare at only $2.7 \%$ (Fig. 10: 7-8). Similar (unpublished) examples occur at Gurga Chiya within the tentatively dated LC2-3 strata, and at Nineveh (Gut 1995: pl. 108: S116). Simple rimmed bowls form 6.7\% of the phase Vd rim sherds (Fig. 10: 3-6), recalling MPBs such as those from Girdi Resh (Hijara 1976: 74), Tepe Gawra VIII (Rothman 2002: fig. 9: n-o), Girdi Qala and Logardan (Vallet et al. 2017: 78), and Nineveh (Gut 1995: pl. 55: 819; 63: 901).

Remaining open forms are represented by single sherds. An incurved rim bowl (Fig. 10: 12) compares with examples from LC2-3 levels at Girdi Resh in the Shahrizor (Hijara 1976: 75), Tepe Gawra VIII (Rothman 2002: pl. 22: 2827), Yorghan Tepe (Starr 1939: fig. 42: J), Leilan IV (Schwartz 1988: fig. 53: 12), and Nineveh (Gut 1995: pl. 107: S66; 108: S81, S92, S95; 109: S130131). Another open form features a flattened, protruding exterior rim (Fig. 10: 2), which compares 


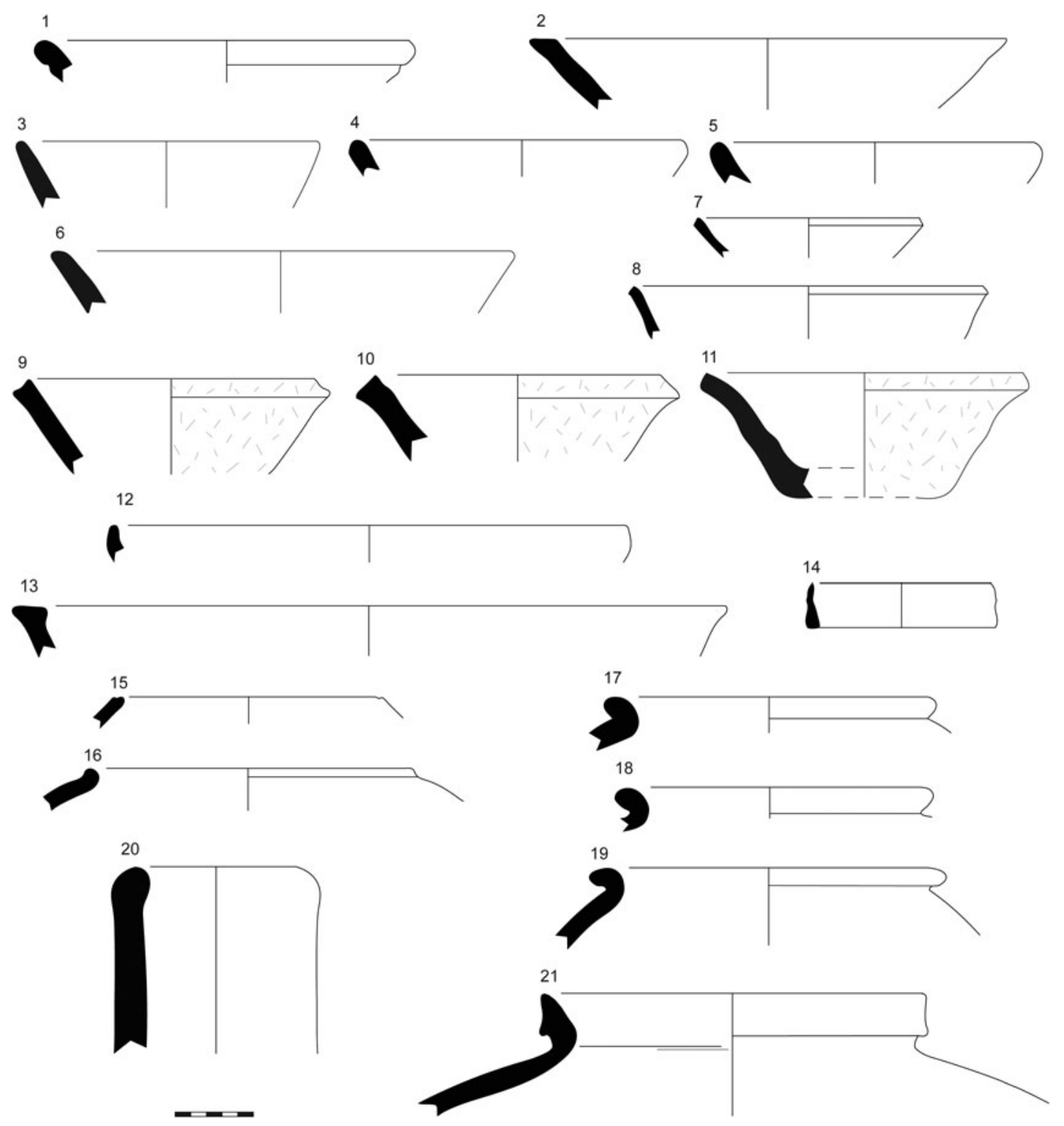

Fig. 10 Phase Vd ceramics: 1-19 and 21: main vessel types; 14: ring scraper; 20: “tuyère”. See Table 7 for details.

well with LC2-3 examples from Girdi Resh in the Shahrizor (Hijara 1976: 77), Leilan IV (Schwartz 1988: fig. 58: 2), and Nineveh (Gut 1995: pl. 113: S271). A similar variant of this has a more pronounced interior rim (Fig. 10: 13). A single example of a club-headed bowl (Fig. 10: 1) compares with examples from Girdi Resh (Hijara 1976: 76), Nineveh (Gut 1995: 248-251), and Hamoukar's Southern Extension (Abu Jayyab 2012: fig. 16: 1).

Closed forms are dominated by globular jars with simple everted rims (10.7\%) (Fig. 10: 17-19), with a single variant that has a more distinctively upright rim, with close parallels at Tepe Gawra VIII (Rothman 2002: pl. 21: 2779), Girdi Resh (Hijara 1976: 78), and Hammam et-Turkman VB (Akkermans 1988: pl. 107: 99-100; 108: 106; 110: 125). Two beaded rim hole-mouth jar fragments (2.6\%) (Fig. 10: 16) have comparative examples at Tell Brak HS6 level 1 (Matthews 2003: fig. 3: 16.6), Hamoukar Area Z (Al-Quntar and Abu Jayyab 2014: fig. 6: 15), and Hammam et-Turkman VB (Akkermans 1988: pl. 107: 101). 
Table 7 Phase Vd: description of Fig. 10 illustrated sherds.

\begin{tabular}{|c|c|c|c|c|c|c|c|c|c|}
\hline No. & Type & Fabric & Technology & $\begin{array}{l}\text { Exterior Surface } \\
\text { Treatment }\end{array}$ & $\begin{array}{l}\text { Interior } \\
\text { Surface } \\
\text { Treatment }\end{array}$ & Exterior Colour & Interior Colour & Core Colour & Slip Colour \\
\hline 1 & club-headed bowl & $\mathrm{F} 2 \mathrm{c}$ & coil / drawn & wet smoothed & wet smoothed & light brown & light brown & very dark grey & \\
\hline 2 & flat rim bowl & $\mathrm{F} 2 \mathrm{c}$ & coil & wet smoothed & wet smoothed & very pale brown & $\begin{array}{l}\text { very pale } \\
\text { brown }\end{array}$ & very pale brown & \\
\hline 3 & simple rim bowl & $\mathrm{F} 2 \mathrm{~b}$ & coil & wet smoothed & wet smoothed & light red & light red & dark grey & \\
\hline 4 & simple rim bowl & $\mathrm{F} 2 \mathrm{~b}$ & coil & wiped & wet smoothed & pink & pink & very dark grey & \\
\hline 5 & simple rim bowl & $\mathrm{F} 2 \mathrm{c}$ & coil & wet smoothed & wet smoothed & pink & pink & dark grey & \\
\hline 6 & simple rim bowl & $\mathrm{F} 2 \mathrm{c}$ & coil & wiped & wet smoothed & $\begin{array}{l}\text { light reddish } \\
\text { brown }\end{array}$ & $\begin{array}{l}\text { light reddish } \\
\text { brown }\end{array}$ & brown & \\
\hline 7 & bowl with beveled rim & $\mathrm{F} 5 \mathrm{c}$ & coil & wet smoothed & wet smoothed & pink & pink & reddish grey & \\
\hline 8 & bowl with beveled rim & $\mathrm{F} 2 \mathrm{c}$ & coil & $\begin{array}{l}\text { wet smoothed } \\
\text { (possibly } \\
\text { slipped) }\end{array}$ & $\begin{array}{l}\text { wet smoothed } \\
\text { (possibly } \\
\text { slipped) }\end{array}$ & very pale brown & $\begin{array}{l}\text { very pale } \\
\text { brown }\end{array}$ & pink & $\begin{array}{l}\text { very pale } \\
\text { brown }\end{array}$ \\
\hline 9 & Beveled Rim Bowl & F1a & mould & $\begin{array}{l}\text { rough, no } \\
\text { treatment }\end{array}$ & wet smoothed & very pale brown & $\begin{array}{l}\text { very pale } \\
\text { brown }\end{array}$ & very pale brown & \\
\hline 10 & Beveled Rim Bowl & F1a & mould & $\begin{array}{l}\text { rough, no } \\
\text { treatment }\end{array}$ & wet smoothed & very pale brown & $\begin{array}{l}\text { very pale } \\
\text { brown }\end{array}$ & grey & \\
\hline 11 & Beveled Rim Bowl & F1a & mould & $\begin{array}{l}\text { rough, no } \\
\text { treatment }\end{array}$ & wet smoothed & very pale brown & $\begin{array}{l}\text { very pale } \\
\text { brown }\end{array}$ & grey & \\
\hline 12 & incurved rim bowl & $\mathrm{F} 2 \mathrm{c}$ & coil & $\begin{array}{l}\text { polished/ } \\
\text { burnished }\end{array}$ & wet smoothed & $\begin{array}{l}\text { light reddish } \\
\text { brown }\end{array}$ & $\begin{array}{l}\text { light reddish } \\
\text { brown }\end{array}$ & dark grey & \\
\hline 13 & $\begin{array}{l}\text { bowl with pronounced int. and } \\
\text { ext. rim }\end{array}$ & $\mathrm{F} 2 \mathrm{c}$ & coil & & burnished & pink & pink & grey & \\
\hline 14 & ring scraper & F1c & & wet smoothed & wet smoothed & pale yellow & pale yellow & pale yellow & \\
\hline 15 & $\begin{array}{l}\text { holemouth jar with grooved } \\
\text { rim }\end{array}$ & $\mathrm{F} 2 \mathrm{c}$ & coil / drawn & burnished & burnished & light red & light red & light red & \\
\hline 16 & beaded rim holemouth jar & $\mathrm{F} 2 \mathrm{c}$ & slab built & wet smoothed & wet smoothed & $\begin{array}{l}\text { light reddish } \\
\text { brown }\end{array}$ & reddish yellow & dark grey & \\
\hline 17 & globular everted rim jar & $\mathrm{F} 5 \mathrm{c}$ & coil & wet smoothed & wet smoothed & pale brown & pale brown & dark grey & \\
\hline 18 & globular everted rim jar & $\mathrm{F} 5 \mathrm{c}$ & coil & wet smoothed & & reddish yellow & reddish yellow & $\begin{array}{l}\text { light yellowish } \\
\text { brown }\end{array}$ & \\
\hline 19 & globular everted rim jar & F5c & coil & burnished & wet smoothed & brown & brown & brown & \\
\hline 20 & tuyère? & $\mathrm{F} 2 \mathrm{~b}$ & slab built & wiped & wet smoothed & light red & light red & reddish yellow & \\
\hline 21 & $\begin{array}{l}\text { angle rim jar with undercut } \\
\text { rim }\end{array}$ & F1b & coil & wet smoothed & wet smoothed & reddish yellow & reddish yellow & very dark grey & \\
\hline
\end{tabular}



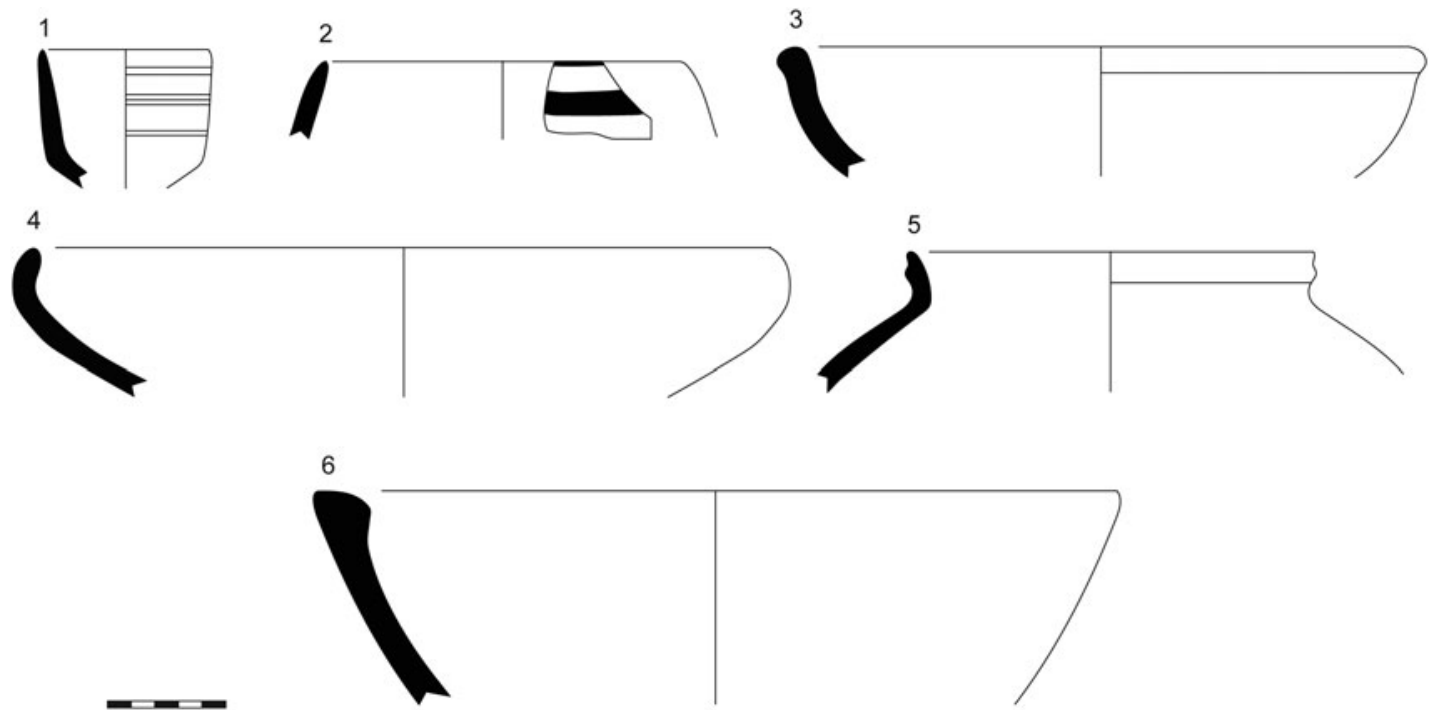

Fig. 11 Phase Vd ceramics from pit. See Table 8 for details.

Remaining closed forms are all documented by single sherds. A jar with a narrow, banded rim and slight concavity (Fig. 10: 21) compares to examples at Hammam et-Turkman VB (Akkermans 1988: pl. 108: 110). A holemouth jar fragment with grooved rim (Fig. 10: 15) has comparative examples from Tell Brak HS1 Level 6 (Felli 2003: fig. 4: 19.15) and Hammam et-Turkman VA (Akkermans 1988: pl. 101: 46), while a narrow closed jar has a slightly thickened rim and is similar to an example at Grai Resh IIB (Kepinski 2011: pl. 11.1).

Two sherds offer glimpses into possible craft production at Kani Shaie. A ring scraper (Fig. 10: 14) is a tool that has been found at numerous contemporary sites and is often associated with pottery manufacture (Alden 1988; Alden \& Minc 2016). One unusual sherd from phase Vd is long with extremely thick walls and a rounded rim and could be part of a tuyère usually associated with metallurgy (Fig. 10: 20), similar to a LC2-4 examples found at Tell Zeidan (Stein 2009: fig. 13) and Hamoukar (Reichel 2008: fig. 6). The use of draft furnaces in the fourth millennium B.C.E. is also well-attested on the Iranian Plateau (Matthews \& Fazeli 2004; Thornton 2009; Weeks 2013).

Phase Vd also includes an additional, separate context: a pit dug into level 11. The forms from this pit include a carinated cup with horizontal combing (Fig. 11:1) with exact parallels at Leilan IV (Schwartz 1988: fig. 53: 2, 4) and Tepe Gawra VIII (Rothman 2002: pl. 23: 2811), a burnished brown-ware incurved rim bowl (Fig. 11: 4) (Rothman 2002: pl. 22: 2827), and a club-headed bowl (Fig. 11: 3). This pit possibly dates slightly later within LC3 than the other material discussed in this section.

\section{Decoration}

Continuing the declining trend of decorated sherds in previous phases, phase $\mathrm{Vd}$ produced no decorated sherds at all.

\section{Fabrics}

Throughout phase V, vegetal tempered fabrics continue to dominate the assemblage with c. $90 \%$ of the diagnostic sherds (Fig. 16). F1a is still the most common fabric group within Phase Vd, at $52 \%$ of total diagnostic sherds, but it was now used exclusively for BRBs. At $30 \%$, F2 continues to grow in importance, becoming the second most common fabric group (F2a 1\%; F2b 5\%; F2c $24 \%$ ). Clearly identifiable mineral tempered sherds only form c. $10 \%$ of diagnostic sherds throughout phase V. Cooking ware fabric F5 is used almost exclusively for simple everted rim jars, with the exception of a single thin-walled bowl with beveled rim and a hole-mouth jar. A new fabric type F7 is attested by a single sherd but could be intrusive from EBA levels. 
TABle 8 Phase Vd-pit: description of Fig. 11 illustrated sherds.

\begin{tabular}{|c|c|c|c|c|c|c|c|c|c|}
\hline No. & Type & Fabric & Technology & $\begin{array}{l}\text { Exterior Surface } \\
\text { Treatment }\end{array}$ & $\begin{array}{l}\text { Interior Surface } \\
\text { Treatment }\end{array}$ & $\begin{array}{l}\text { Exterior } \\
\text { Colour }\end{array}$ & $\begin{array}{l}\text { Interior } \\
\text { Colour }\end{array}$ & Core Colour & $\begin{array}{l}\text { Decoration } \\
\text { Colour }\end{array}$ \\
\hline 1 & cup & F1c & $\begin{array}{l}\text { coil and wheel } \\
\text { finish }\end{array}$ & $\begin{array}{l}\text { wet smoothed and } \\
\text { polished }\end{array}$ & wet smoothed & pale brown & pale brown & pale brown & \\
\hline 2 & painted holemouth jar & F1c & coil / drawn & wet smoothed & wet smoothed & pale brown & pale brown & pale brown & strong brown \\
\hline 3 & club headed bowl & F1c & coil & $\begin{array}{l}\text { wet smoothed and } \\
\text { burnished }\end{array}$ & $\begin{array}{l}\text { wet smoothed and } \\
\text { burnished }\end{array}$ & $\begin{array}{l}\text { reddish } \\
\text { yellow }\end{array}$ & $\begin{array}{l}\text { reddish } \\
\text { yellow }\end{array}$ & $\begin{array}{l}\text { very dark } \\
\text { grey }\end{array}$ & \\
\hline 4 & incurved rim bowl & $\mathrm{F} 1 \mathrm{~b}$ & coil & $\begin{array}{l}\text { wet smoothed and } \\
\text { burnished }\end{array}$ & $\begin{array}{l}\text { wet smoothed and } \\
\text { burnished }\end{array}$ & pink & $\begin{array}{l}\text { reddish } \\
\text { yellow }\end{array}$ & dark grey & \\
\hline 5 & $\begin{array}{l}\text { angle rim jar with } \\
\text { undercut rim }\end{array}$ & F1b & coil / drawn & $\begin{array}{l}\text { wet smoothed and } \\
\text { burnished }\end{array}$ & $\begin{array}{l}\text { wet smoothed and } \\
\text { burnished }\end{array}$ & $\begin{array}{l}\text { reddish } \\
\text { yellow }\end{array}$ & $\begin{array}{l}\text { reddish } \\
\text { yellow }\end{array}$ & $\begin{array}{l}\text { very dark } \\
\text { grey }\end{array}$ & \\
\hline 6 & $\begin{array}{l}\text { internally beveled rim } \\
\text { bowl }\end{array}$ & F1c & coil & wet smoothed & wet smoothed & $\begin{array}{l}\text { very pale } \\
\text { brown }\end{array}$ & $\begin{array}{l}\text { very pale } \\
\text { brown }\end{array}$ & $\begin{array}{l}\text { very pale } \\
\text { brown }\end{array}$ & \\
\hline
\end{tabular}


Forming Techniques and Surface Treatments

Many sherds from phase Vd are small and only preserve the rim, which does not allow the identification of the complete production process of the original vessels. The small number of sherds that does allow such an assessment might not represent the full range of forming techniques that were used during this period. The crackled exterior and finger impressions and smoothing on the interior indicate that BRBs were mould-made, most likely within an existing BRB (McAdam and Mynors 1988: 40; Miller 1981: 128; Nissen 1970: 137). The remainder of the sherds from phase Vd show evidence of manufacture via coiling. Most of the sherds show evidence for finishing on a rotary device or tournette, with final wet smoothing or wiping of the vessels. Additional surface treatment is relatively rare. Only four sherds show evidence of burnishing, though this was not particular to any specific vessel form.

\section{Phase Vc}

Phase Vc is the most pottery-dense phase at Kani Shaie with a total of 1010 sherds, of which 167 were diagnostic (16.5\%), including 133 rim sherds ( $13.2 \%$ of the total sherd count). The remaining diagnostic sherds were bases, handles, spouts, and decorated sherds. 75 bowl fragments amount to $56.4 \%$ of the rim sherd count, while jars are represented by 48 rim sherds (36.1\%), and cups by 10 sherds $(7.5 \%)$.

\section{Forms}

BRBs continue to dominate the phase Vc assemblage, but only at $21 \%$ of the rim sherd count, a lower quantity compared to $57.3 \%$ in the previous phase Vd (Fig. 12: 7). Such an extreme fluctuation was also observed at Tell Rubeidheh, where BRB quantities range even more, from as little as $15 \%$ to $>70 \%$ (McAdam and Mynors 1988: 40-41).

Simple bowls with wide, flaring rims continue into phase Vc (16.5\%) (Fig. 12: 4-6). Similar bowls occur in LC4 strata at Tell Rubeidheh (McAdam and Mynors 1988: fig. 28: 11), Tell Hassan (Nannucci 2012: fig. 2: 5-10, 13-16), Hacinebi (Pearce 2000: fig. 13: b-c), and Sheikh Hassan (Boese 1995: fig. 9: a, fig. 13: a). Thin-walled bowls with a beveled rim continue in low numbers $(3 \%)$ into the lower level of phase Vc, but not beyond (Fig. 12: 8-10).

Conical cups with thin walls and a string-cut base occur for the first time in phase Vc, forming $6.8 \%$ of the total rim sherd count (Fig. 12: 1-3). Only one example from this total featured a pouring lip, but see below for additional examples assigned to this phase. Such cups are very common at Uruk period sites, with nearby comparative examples from LC4 strata at Gurga Chiya (Wengrow et al. 2016: fig. 8: 12), Tell Rubeidheh (McAdam and Mynors 1988: fig. 28: 6), Ahmad al-Hattu (Sürenhagen 1979: fig. 4), and Tell Hassan (Nannucci 2012: fig. 2: 3-4).

Incurved-rim bowls, which are a common and wide-spread form within LC3-5 assemblages throughout Mesopotamia, increase from 3\% to 7.5\% (Fig. 12: 11-14). Within the vicinity of Kani Shaie, they are well-documented at Gurga Chiya (Carter et al. 2020) in the Shahrizor, and at Tell Hassan (Nannucci 2012: fig. 3: 27-30) and Tell Rubeidheh (McAdam and Mynors 1988: fig. 28: 10) in the Hamrin basin, where they are noted as amongst the most common form of bowl (ibid: 45). They also occur in the central Zagros at Godin Tepe (Rothman and Badler 2011: fig. 4: 45.1c).

Open vessels found in lower quantities include club-headed bowls with flattened or slightly oblique tops and pronounced interior and exterior rims (3\%) (Fig. 12: 16-17), which at present occur exclusively in the earliest level of Phase Vc. They closely resemble LC3 hammerhead bowls, though the form at Kani Shaie features a flat-topped rim rather than the more characteristic oblique profiled rims. Similar examples are documented at Girdi Resh (Hijara 1976: 76), Leilan IV (Schwartz 1988: fig. 52: 5), and Nineveh (Gut 1995: pl. 113: S283-284).

Bowls with a flat, square profile rim total 1.5\% within phase Vc (Fig. 12: 18-19) and have parallels in LC2-3 strata at Hammam et-Turkman VB (Akkermans 1988: pl. 104: 79) and slightly later within early LC4 phases at Leilan IV (Schwartz 1988: fig. 53: 3, 5). Other open forms from phase Vc represented only by single sherds include a bowl with a slight carination to the shoulder (Fig. 12: 15) closely resembling an example from Tepe Gawra VIII (Rothman 2002: pl. 22: 2825). Another sherd belongs to an open bowl with a flattened, protruding exterior rim, with similar LC2-3 examples from Girdi Resh (Hijara 1976: 77) and Nineveh (Gut 1995: pl. 113: S271). A variant of this type has a more pronounced interior lip. 


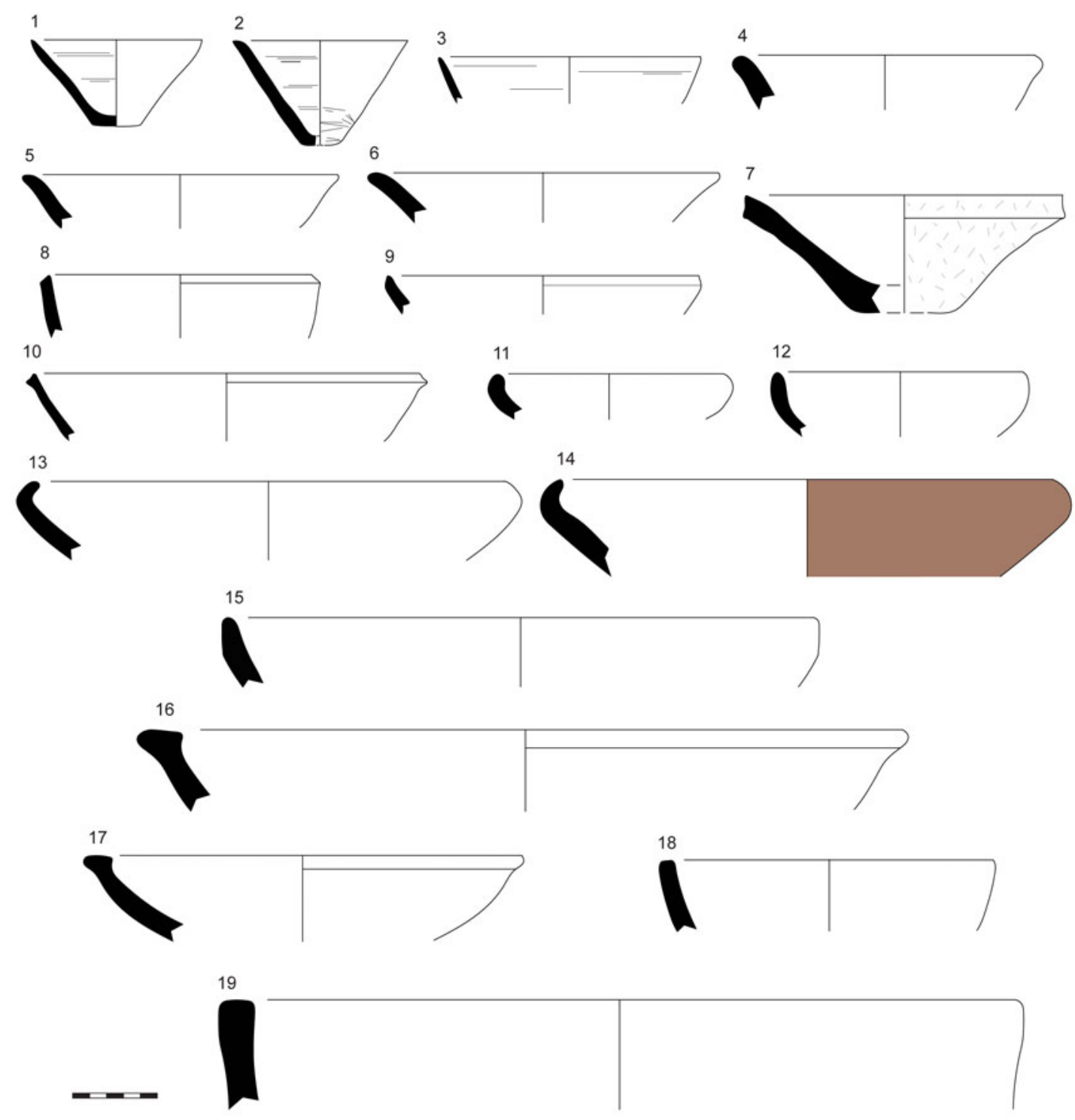

Fig. 12 Phase Vc ceramics: open shapes. See Table 9 for details.

Globular jars with simple, everted rims remain the most common closed shape in phase $\mathrm{Vc}(9 \%)$ (Fig. 13: 1-4), with parallels at nearby LC4 sites including Gurga Chiya (Wengrow et al. 2016: fig. 8: 4-5), Tell Rubeidheh (McAdam and Mynors 1988: fig. 30: 41, 45), Ahmad al-Hattu (Sürenhagen 1979: fig. 10), and Tell Hassan (Nannucci 2012: fig. 5: 58, 60-63). Angled rim jars with an undercut rim (5.2\%) become prevalent in phase Vc (Fig. 13: 9-14). Some examples feature a slight concavity to the band rim. These jar rims are triangular in profile with a sharply carinated neck, ideal as a support for a lid. Such vessels are well-known from LC4 pottery assemblages at Gurga Chiya (Wengrow et al. 2016: fig. 8: 6, 8), Tell Rubeidheh (McAdam and Mynors 1988: fig. 31: 5760), and Tell Hassan (Nannucci 2012: fig. 6: 75-81), as well as at Sheikh Hassan (Boese 1995: 201, fig. 13: 1-n) in western Syria. Necked jars with flat-topped, everted or ledge rims (Fig. 13: 5-8) compare to similar forms from LC4 levels at Tell Hassan (Nannucci 2012: fig. 8: 108-110), Tell Rubeidheh (McAdam and Mynors 1988: fig. 32: 80), and Chogha Mish (Delougaz et al. 1996: pl. 91: I). This form is less common at only $4.5 \%$, but it seems to represent a chronologically sensitive type considering that with only one exception, all examples of these vessels come from phase Vc, 
TABle 9 Phase Vc open shapes: description of Fig. 12 illustrated sherds.

\begin{tabular}{|c|c|c|c|c|c|c|c|c|c|}
\hline No. & Type & Fabric & Technology & $\begin{array}{l}\text { Exterior Surface } \\
\text { Treatment }\end{array}$ & $\begin{array}{l}\text { Interior } \\
\text { Surface } \\
\text { Treatment }\end{array}$ & $\begin{array}{l}\text { Exterior } \\
\text { Colour }\end{array}$ & Interior Colour & Core Colour & Slip Colour \\
\hline 1 & conical cup & $\mathrm{F} 2 \mathrm{c}$ & wheel made & wet smoothed & wet smoothed & reddish yellow & $\begin{array}{l}\text { light yellowish } \\
\text { brown }\end{array}$ & $\begin{array}{l}\text { light yellowish } \\
\text { brown }\end{array}$ & \\
\hline 2 & conical cup & $\mathrm{F} 2 \mathrm{c}$ & wheel made & $\begin{array}{l}\text { wet smoothed, } \\
\text { scraped bottom }\end{array}$ & wet smoothed & reddish yellow & very pale brown & very pale brown & \\
\hline 3 & conical cup & F1c & coil & wet smoothed & wet smoothed & $\begin{array}{l}\text { very pale } \\
\text { brown }\end{array}$ & very pale brown & very pale brown & \\
\hline 4 & simple rim bowl & $\mathrm{F} 2 \mathrm{c}$ & coil & wet smoothed & wet smoothed & pink & pink & pink & \\
\hline 5 & simple rim bowl & $\mathrm{F} 2 \mathrm{c}$ & coil & self slip and wiped & $\begin{array}{l}\text { self slip and } \\
\text { wiped }\end{array}$ & light red & light red & $\begin{array}{l}\text { light reddish } \\
\text { brown }\end{array}$ & \\
\hline 6 & simple rim bowl & $\mathrm{F} 2 \mathrm{c}$ & coil & wet smoothed & $\begin{array}{l}\text { self slip and } \\
\text { wiped }\end{array}$ & pink & pink & pink & \\
\hline 7 & Beveled Rim Bowl & F1a & mould & rough & wet smoothed & light grey & light grey & light grey & \multirow{13}{*}{$\begin{array}{l}\text { reddish } \\
\text { yellow } \\
\text { pinkish } \\
\text { grey }\end{array}$} \\
\hline 8 & bowl with beveled rim & F1c & coil & wet smoothed & wet smoothed & pale brown & pale brown & pale brown & \\
\hline 9 & bowl with beveled rim & $\mathrm{F} 2 \mathrm{c}$ & coil & wet smoothed & burnished? & $\begin{array}{l}\text { light reddish } \\
\text { brown }\end{array}$ & light red & $\begin{array}{l}\text { light reddish } \\
\text { brown }\end{array}$ & \\
\hline 10 & bowl with beveled rim & $\mathrm{F} 2 \mathrm{c}$ & coil & burnished? & burnished? & $\begin{array}{l}\text { very pale } \\
\text { brown }\end{array}$ & light red & very pale brown & \\
\hline 11 & incurved rim bowl & $\mathrm{F} 2 \mathrm{c}$ & drawn & wet smoothed & wet smoothed & pale yellow & pale yellow & pale yellow & \\
\hline 12 & incurved rim bowl & $\mathrm{F} 2 \mathrm{c}$ & & wet smoothed & wet smoothed & reddish yellow & reddish yellow & reddish yellow & \\
\hline 13 & incurved rim bowl & $\mathrm{F} 2 \mathrm{c}$ & coil / drawn & self slip & self slip & pink & reddish yellow & $\begin{array}{l}\text { light brownish } \\
\text { grey }\end{array}$ & \\
\hline 14 & incurved rim bowl & $\mathrm{F} 2 \mathrm{c}$ & coil & slipped & slipped & reddish yellow & reddish yellow & grey & \\
\hline 15 & $\begin{array}{l}\text { bowl with carinated } \\
\text { shoulder }\end{array}$ & $\mathrm{F} 2 \mathrm{c}$ & coil & slipped & slipped & pinkish grey & grey & grey & \\
\hline 16 & $\begin{array}{l}\text { club-headed bowl with } \\
\text { flat rim }\end{array}$ & $\mathrm{F} 2 \mathrm{~b}$ & coil & scraped below rim & $\begin{array}{l}\text { self slip and } \\
\text { wiped }\end{array}$ & reddish yellow & reddish yellow & grey & \\
\hline 17 & $\begin{array}{l}\text { club-headed bowl with } \\
\text { flat rim }\end{array}$ & $\mathrm{F} 2 \mathrm{~b}$ & coil / drawn & self slip & $\begin{array}{l}\text { self slip / } \\
\text { burnished }\end{array}$ & reddish yellow & reddish yellow & dark grey & \\
\hline 18 & $\begin{array}{l}\text { flattened rim bowl with } \\
\text { square profile }\end{array}$ & $\mathrm{F} 2 \mathrm{c}$ & $\begin{array}{l}\text { drawn body, } \\
\text { coil rim }\end{array}$ & burnished rim & burnished rim & reddish yellow & reddish yellow & reddish yellow & \\
\hline 19 & $\begin{array}{l}\text { flattened rim bowl with } \\
\text { square profile }\end{array}$ & $\mathrm{F} 2 \mathrm{c}$ & coil & wet smoothed & wet smoothed & light brown & light brown & grey & \\
\hline
\end{tabular}




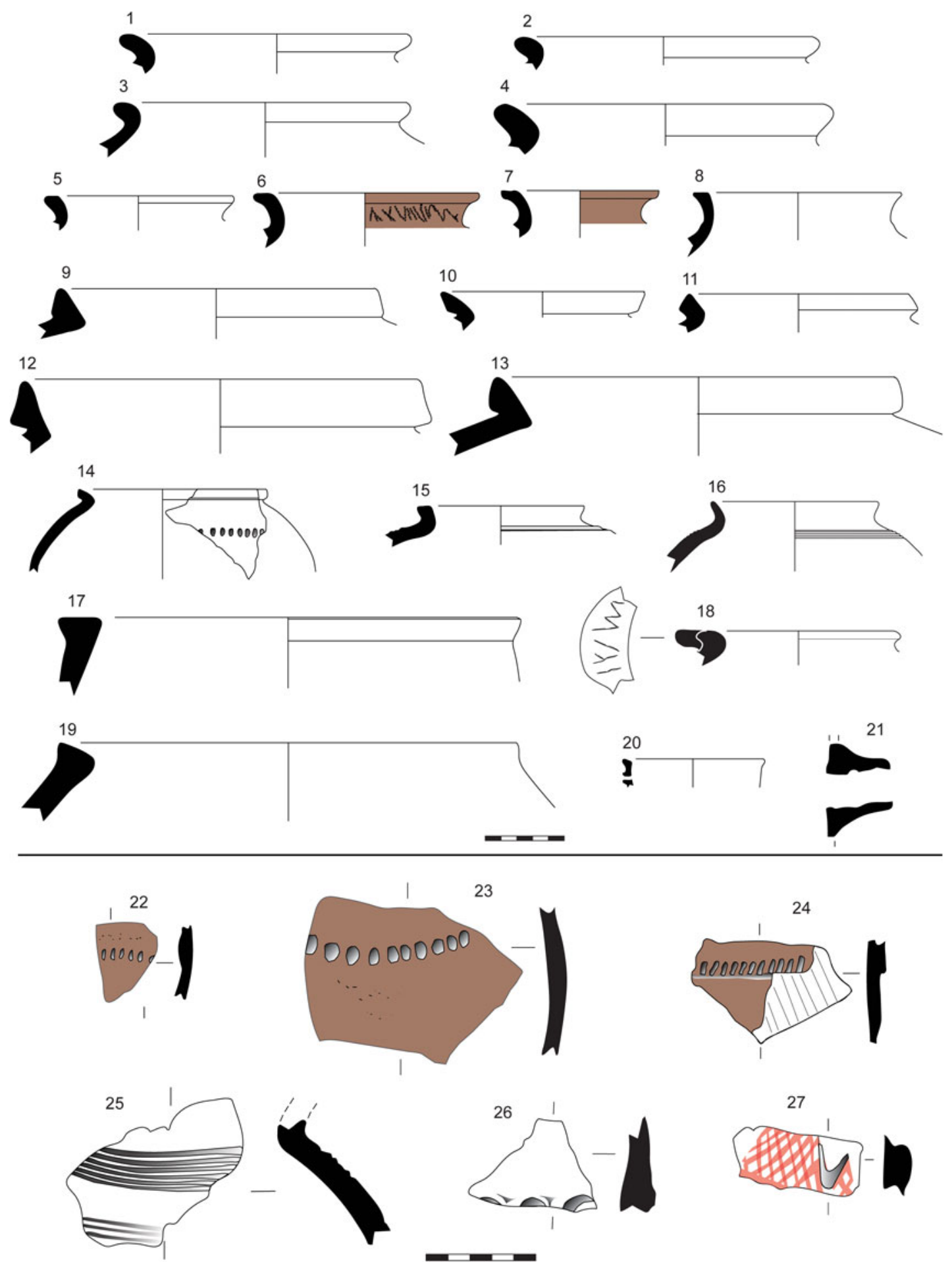

Fig. 13 Phase Vc ceramics: closed shapes. See Table 10 for details.

particularly the earlier level. Neckless jars with everted, flat topped rims are exclusive to this phase $(1.5 \%)$ (Fig. 13: 17). Comparative examples are known from Tell Rubeidheh (McAdam and Mynors 1988: fig. 29: 33) and Sheikh Hassan (Boese 1995: 227, fig. 13: 1-b), while a similar vessel from Tell Hassan has a slightly different rim (Nannucci 2012: fig. 5: 43). 
TABLE 10 Phase Ve closed shapes: description of Fig. 13 illustrated sherds.

\begin{tabular}{|c|c|c|c|c|c|c|c|c|c|}
\hline No. & Type & Fabric & Technology & $\begin{array}{l}\text { Exterior Surface } \\
\text { Treatment }\end{array}$ & $\begin{array}{l}\text { Interior Surface } \\
\text { Treatment }\end{array}$ & $\begin{array}{l}\text { Exterior } \\
\text { Colour }\end{array}$ & $\begin{array}{l}\text { Interior } \\
\text { Colour }\end{array}$ & Core Colour & Slip Colour \\
\hline 1 & globular everted rim jar & F5c & coil / drawn & wet smoothed & wet smoothed & $\begin{array}{l}\text { light reddish } \\
\text { brown }\end{array}$ & $\begin{array}{l}\text { light reddish } \\
\text { brown }\end{array}$ & $\begin{array}{l}\text { light reddish } \\
\text { brown }\end{array}$ & \\
\hline 2 & globular everted rim jar & $\mathrm{F} 2 \mathrm{c}$ & coil & & & pink & pink & very dark grey & \\
\hline 3 & globular everted rim jar & F6 & coil & burnished & wet smoothed & grey & grey & very dark grey & \\
\hline 4 & globular everted rim jar & $\mathrm{F} 2 \mathrm{c}$ & coil & self slip & self slip & reddish yellow & reddish yellow & reddish yellow & \\
\hline 5 & necked jar with flat rim & $\mathrm{F} 2 \mathrm{c}$ & coil / drawn & wet smoothed & wet smoothed & $\begin{array}{l}\text { very pale } \\
\text { brown }\end{array}$ & $\begin{array}{l}\text { very pale } \\
\text { brown }\end{array}$ & reddish yellow & \\
\hline 6 & necked jar with flat rim & $\mathrm{F} 2 \mathrm{c}$ & coil & $\begin{array}{l}\text { slipped and pattern } \\
\text { burnished }\end{array}$ & $\begin{array}{l}\text { slipped and } \\
\text { burnished }\end{array}$ & reddish yellow & reddish yellow & reddish yellow & $\begin{array}{l}\text { reddish } \\
\text { yellow }\end{array}$ \\
\hline 7 & necked jar with flat rim & $\mathrm{F} 2 \mathrm{c}$ & coil & slipped & $\begin{array}{l}\text { slipped (only } \\
\text { rim) }\end{array}$ & pale brown & pale yellow & pink & pale brown \\
\hline 8 & necked jar with flat rim & $\mathrm{F} 2 \mathrm{c}$ & coil & self slip & wet smoothed & light red & pink & $\begin{array}{l}\text { light reddish } \\
\text { brown }\end{array}$ & \\
\hline 9 & $\begin{array}{l}\text { angle rim jar with } \\
\text { undercut rim }\end{array}$ & $\mathrm{F} 2 \mathrm{c}$ & coil & slipped & wet smoothed & pinkish grey & pink & grey & $\begin{array}{l}\text { pinkish } \\
\text { grey }\end{array}$ \\
\hline 10 & $\begin{array}{l}\text { angle rim jar with } \\
\text { undercut rim }\end{array}$ & $\mathrm{F} 2 \mathrm{c}$ & coil & wet smoothed & wet smoothed & reddish yellow & reddish yellow & reddish yellow & \\
\hline 11 & $\begin{array}{l}\text { angle rim jar with } \\
\text { undercut rim }\end{array}$ & $\mathrm{F} 2 \mathrm{~b}$ & coil (?) & wet smoothed & wet smoothed & $\begin{array}{l}\text { very pale } \\
\text { brown }\end{array}$ & $\begin{array}{l}\text { very pale } \\
\text { brown }\end{array}$ & $\begin{array}{l}\text { very pale } \\
\text { brown }\end{array}$ & \\
\hline 12 & $\begin{array}{l}\text { angle rim jar with } \\
\text { undercut rim }\end{array}$ & $\mathrm{F} 2 \mathrm{~b}$ & coil & self slip and wiped & $\begin{array}{l}\text { self slip and } \\
\text { wiped }\end{array}$ & reddish yellow & reddish yellow & grey & \\
\hline 13 & $\begin{array}{l}\text { angle rim jar with } \\
\text { undercut rim }\end{array}$ & $\mathrm{F} 2 \mathrm{~b}$ & coil & self slip and wiped & $\begin{array}{l}\text { self slip and } \\
\text { wiped }\end{array}$ & reddish yellow & reddish yellow & grey & \\
\hline 14 & $\begin{array}{l}\text { angle rim jar with } \\
\text { undercut rim }\end{array}$ & $\mathrm{F} 2 \mathrm{c}$ & coil / drawn & burnished & self slip & pale brown & pale brown & pale brown & \\
\hline 15 & $\begin{array}{l}\text { flat rim jar with horizontal } \\
\text { combing }\end{array}$ & $\mathrm{F} 2 \mathrm{c}$ & coil & wet smoothed & & reddish yellow & reddish yellow & reddish yellow & \\
\hline 16 & $\begin{array}{l}\text { angle neck jar with } \\
\text { horizontal combing }\end{array}$ & F6 & coil / drawn & wet smoothed & wet smoothed & light brown & light brown & pale brown & \\
\hline 17 & $\begin{array}{l}\text { neckless jar with everted } \\
\text { flattened rim }\end{array}$ & $\mathrm{F} 2 \mathrm{c}$ & coil / drawn & wet smoothed & scraped interior & reddish yellow & reddish yellow & grey & \\
\hline 18 & $\begin{array}{l}\text { everted rim jar with rim } \\
\text { swelling }\end{array}$ & F6 & coil & wet smoothed & wet smoothed & $\begin{array}{l}\text { light reddish } \\
\text { brown }\end{array}$ & $\begin{array}{l}\text { light reddish } \\
\text { brown }\end{array}$ & pink & \\
\hline 19 & jar with narrowed rim & $\mathrm{F} 2 \mathrm{c}$ & coil / drawn & $\begin{array}{l}\text { wet smoothed and } \\
\text { scraped below rim }\end{array}$ & $\begin{array}{l}\text { self slip and } \\
\text { wiped }\end{array}$ & reddish yellow & reddish yellow & grey & \\
\hline
\end{tabular}


TABLE 10 (Continued)

\begin{tabular}{|c|c|c|c|c|c|c|c|c|c|}
\hline No. & Type & Fabric & Technology & $\begin{array}{l}\text { Exterior Surface } \\
\text { Treatment }\end{array}$ & $\begin{array}{l}\text { Interior Surface } \\
\text { Treatment }\end{array}$ & $\begin{array}{l}\text { Exterior } \\
\text { Colour }\end{array}$ & $\begin{array}{l}\text { Interior } \\
\text { Colour }\end{array}$ & Core Colour & Slip Colour \\
\hline 20 & small vessel w. spout & $\mathrm{F} 2 \mathrm{c}$ & coil & wet smoothed & wet smoothed & pink & pink & pink & \\
\hline 21 & spout & F6 & $\begin{array}{l}\text { applied } \\
\text { spout }\end{array}$ & wet smoothed & wet smoothed & pink & pink & pink & \\
\hline 22 & $\begin{array}{l}\text { body sherd with incised } \\
\text { cordon }\end{array}$ & $\mathrm{F} 2 \mathrm{c}$ & coil & slipped & wet smoothed & pink & pink & pink & red \\
\hline 23 & $\begin{array}{l}\text { body sherd with impressed } \\
\text { cordon }\end{array}$ & $\mathrm{F} 2 \mathrm{c}$ & coil & wet smoothed & slipped & reddish yellow & reddish yellow & reddish yellow & $\begin{array}{l}\text { reddish } \\
\text { yellow }\end{array}$ \\
\hline 24 & $\begin{array}{l}\text { body sherd with applique } \\
\text { cordon }\end{array}$ & F1c & coil & wet smoothed & slipped & reddish yellow & reddish yellow & reddish yellow & red \\
\hline 25 & comb incised body sherd & $\mathrm{F} 2 \mathrm{c}$ & coil / drawn & wet smoothed & wet smoothed & light brown & light brown & pale brown & \\
\hline 26 & $\begin{array}{l}\text { body sherd with rope } \\
\text { cordon }\end{array}$ & $\mathrm{F} 2 \mathrm{a}$ & coil & wet smoothed & wet smoothed & light grey & light grey & light grey & \\
\hline 27 & $\begin{array}{l}\text { painted body sherd with } \\
\text { nose lug }\end{array}$ & F1c & coil & wet smoothed & wiped & pink & pink & pink & $\begin{array}{l}\text { paint: light } \\
\text { red }\end{array}$ \\
\hline
\end{tabular}




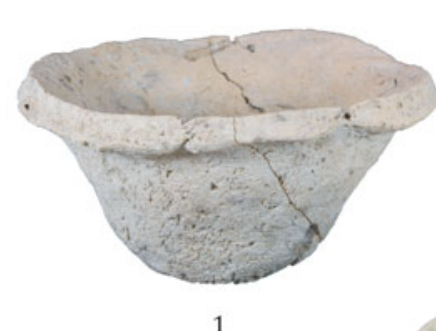

1

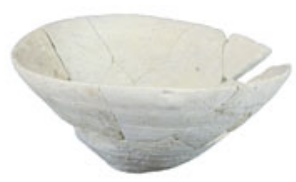

2

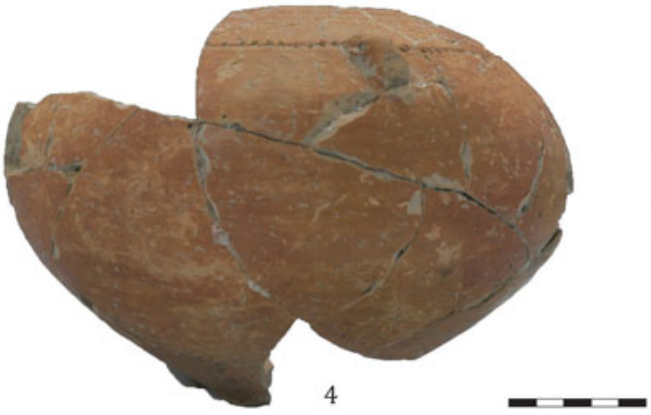

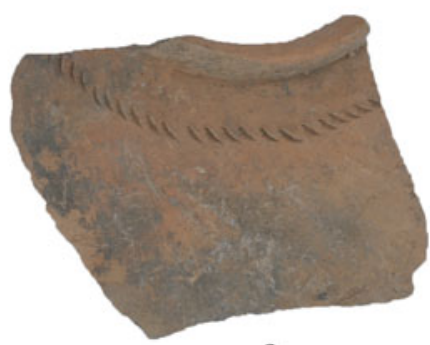

3

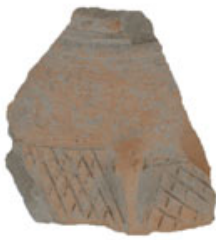

5

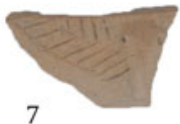

Fig. 14 Phase Vc "Uruk" pottery: 1: BRB; 2: conical cup with string-cut base; 3: jar with fingernail impressions; 4-7: nose-lug jar sherds.

Remaining forms are rare within phase Vc. Jars with narrowed, very short rims (1.5\%) (Fig. 13: 19-20) have close parallels at Tell Rubeidheh (McAdam and Mynors 1988: fig. 29: 28-29). Another jar represented by a single documented sherd, unique to this phase, features an upright and flattened rim (Fig. 13: 15) with a comparison at Tell Hassan (Nannucci 2012: fig. 8: 105). The example from Kani Shaie is decorated with a horizontal incised band around the shoulder and is characterised by a large quantity of angular mineral temper.

Additional diagnostic forms not included within the above counts include two cannon spouts (Fig. 13: 21) and sherds with rim swellings, or blunt, flattened lips with incised scoring (Fig. 13: 18) that have comparanda at Tell Rubeidheh (McAdam and Mynors 1988: fig. 34: 97) and Chogha Mish (Delougaz et al. 1996: pl. 98: H-K). Two body sherds with nose lugs find nearby comparisons at Gurga Chiya (Wengrow et al. 2016: fig. 8: 7), Tell Rubeidheh (McAdam and Mynors 1988: fig. 34: 98-104), and Tell Hassan (Nannucci 2012: fig. 9: 139, 10: 152-155), but are otherwise well-documented throughout Mesopotamia.

Three contexts assigned to phase $\mathrm{Vc}$ have not been included within the above tallies because they are stratigraphically disconnected from the other contexts. Based on elevations, stratigraphic analysis, and material culture, these contexts clearly belong to phase $\mathrm{Vc}$, but their ceramic assemblage shows some clear differences that are not yet fully understood (Fig. 14). BRBs dominate the assemblage from these contexts, while thin-walled conical cups with string-cut bases are also found in very high quantities in these three contexts, both with and without a pouring lip. Other open forms include incurved rim bowls and wide bowls with flattened, protruding interior rims. Closed forms include red-slipped jars with nose lugs and incised rope cordons, globular jars with simple, everted rims, and jars with triangular profiles and sharply carinated necks. Overall this small sub-assemblage compares well with the broader phase Vc as well as the LC4 assemblages from Gurga Chiya, Tell Rubeidheh, Ahmad al-Hattu, and Tell Hassan, but it contains a much higher ratio of BRBs and thin-walled conical cups than the main phase Vc levels, as well as a much more 
restricted range of shapes. Whether these differences reflect chronological or functional differences between these contexts remains to be determined.

\section{Decoration}

19 sherds from phase Vc have decoration (6 painted; 13 incised/impressed); only three of these are rim sherds. This total equates to $11.4 \%$ of the total diagnostic sherd count and $1.9 \%$ of the total sherd count. One sherd features a reddish-brown painted design of a cross-hatched triangular motif around the shoulder, with traces of a lug handle (Fig. 13: 27). Similar nose-lug jars with painted criss-cross motifs are known from Late Uruk and Jemdet Nasr strata from Central Mesopotamia (Rova 2014: 4).

Incised/impressed decoration represents $1.3 \%$ of the total sherd count, or $7.8 \%$ of diagnostic sherds. Several sherds of closed jars have horizontal combing on the exterior (Fig. 13: 15-16, 25). Similar examples are depicted from Tell Brak HS1 (Felli 2003: fig. 4: 25.11) and Sheikh Hassan (Boese 1995: 83, fig. 20: a-e), as well as from Susa Acropole I:17 (Le Brun 1978: fig. 34: 12) and Chogha Mish (Delougaz et al. 1996: pl. 95: K-N). One body sherd has thick incised parallel lines with much thinner incised lines running perpendicular, comparable to a decorative technique seen at Chogha Mish (Delougaz et al. 1996: pl. 101: E). Rope cordons are also relatively common decorative motifs (Fig. 13: 26). Simple applications include vertical slashes to the shoulder of the vessel (Fig. 13: 22), impressed thumb-print design to the vessel shoulder (Fig. 13: 23), and an applied band of clay with impressed thumb nail design over the top (Fig. 13: 24). One rim sherd has an impressed thumbnail design with an incised horizontal band on the shoulder of the vessel (McAdam and Mynors 1988: fig 36: 123-4).

\section{Fabrics}

In phase Vc, F2 becomes the dominant fabric group with $58 \%$ of diagnostic sherds (F2a $2 \%$; F2b $12 \%$; F2c 44\%), a considerable increase from phase Vd (Fig. 16). F1a decreases substantially from $52 \%$ to $20 \%$, but this reflects the significantly lower amount of BRBs in the phase Vc assemblage. The finer fabric F1c, on the other hand, remains relatively constant at $10 \%$. A new fabric type F8, which is very fine with only extremely fine, sand-sized mica inclusions visible, is observed in a single sherd.

Remaining fabric groups show the addition of mineral temper. Cooking ware fabric F5c forms $6 \%$ of diagnostic sherds and is still primarily used for everted rim globular jars, although isolated examples of other jar forms also occur. A new fabric type F6, which features the deliberate addition of chert, is attested in $3 \%$ of the diagnostic sherds.

Forming Techniques and Surface Treatments

Coiling continues to be the dominant method of ceramic manufacture (58.7\%). The rough-out vessel was then drawn and manipulated by the potter using a tournette or similar rotary device with final wet smoothing or wiping of the vessels. Typical thin-walled conical cups have clear evidence for horizontal rill-marks around the vessel and a string-cut base, demonstrative of manufacture on a fast wheel. A trend in the manufacturing of these cups can be observed at Kani Shaie. Three conical cups from the earlier level of phase $\mathrm{Vc}$ feature a flint scraped lower body without a stringcut base, a practice reminiscent of the scraping of the bases of MPBs to remove excess clay. It seems that this represents an early variant of the string-cut conical cups noted in late phase Vc and $\mathrm{Va}-\mathrm{b}$ that never have this scraping. These distinctive cups match examples from "Early Uruk" levels at Logardan (Baldi 2017: 57).

Pinched and hand-drawn vessels are very rare, and only two sherds show this forming technique for the body. Slipping of sherds was rare in phase $\mathrm{Vc}$ and is not particular to any type or form of vessel. Two distinct slip colours were observed: the first has a red/reddish-yellow colour, while the second, much rarer, slip was grey. Burnishing is also observed, which again is not specific to any type of vessel, but it is more common on closed vessels and vessels that have been slipped. One sherd features a unique pattern burnish and shows a zig-zag pattern applied to the neck of a closed, ledge-rim jar. This rocker pattern is usually applied on squat jars, often spouted or with a strap 


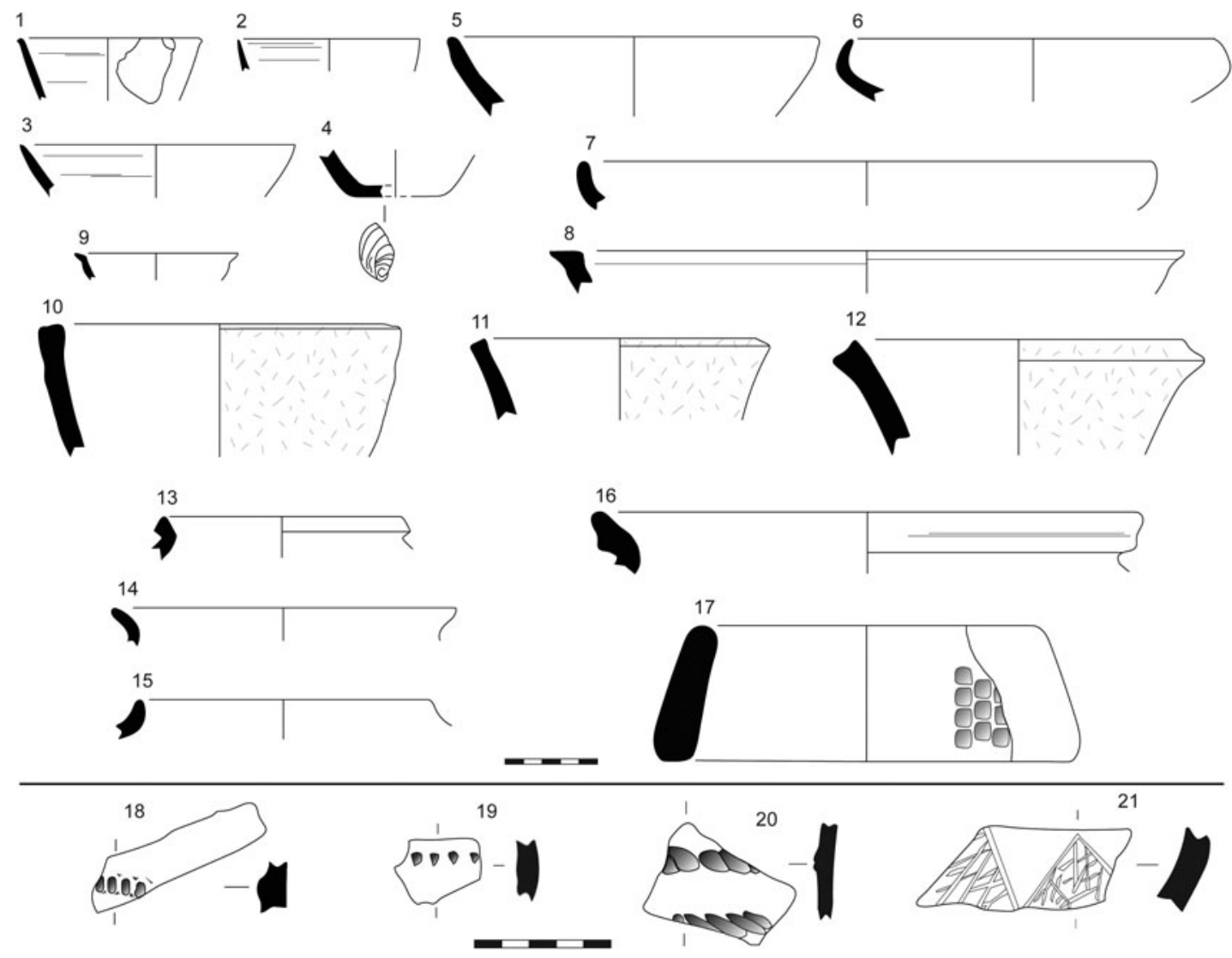

Fig. 15 Phase Va-b ceramics. See Table 11 for details.

handle, at Tell Rubeidheh (McAdam and Mynors 1988: fig. 29: 34, 33: 87-88, 35: 105) and Tell Hassan (Nanucci 2012: fig. 10: 145) in the Hamrin, as well as at Chogha Mish (Alizadeh 2009: fig. 32: K; Delougaz et al. 1996: pl. 107: H-I).

\section{Phase Va-b}

957 sherds were registered from these contexts, of which only 50 were diagnostic (5.2\%) whilst 40 were rim sherds (4.2\%). Many of the non-diagnostic sherds were very small fragments, which could be a result of a hiatus in occupation and exposure to the elements. The phase Va-b assemblage is dominated by bowls $(65 \%)$. Cups amount to $12.5 \%$, while closed forms total $22.5 \%$.

\section{Forms}

Overall the range of forms in phase $\mathrm{Va}-\mathrm{b}$ is very limited and probably not fully representative, but it continues the phase $\mathrm{Vc}$ assemblage with few additions. BRBs continue to be the most abundant form at $37.5 \%$ of the total rim sherd count (Fig. 15: 10-12). Late examples of BRBs from Kani Shaie are characterised by a pink coloured fabric and noticeably taller profile, with contemporary parallels at Mohammed 'Arab (Roaf and Killick 1987: 207), Telul eth-Thalathat (Numoto 1998: 53-4), and Susa Acropole I (Le Brun 1971: fig. 60: 12, 65: 7). Thin-walled conical cups with string-cut bases also continue and total $12.5 \%$ of the phase $\mathrm{Va}-\mathrm{b}$ rim sherd count, including examples with a pouring lip (Fig. 15: 1-4). Similarly, incurved-rim bowls still form 7.5\% (Fig. 15: 6-7) and simple rim bowls form 5\% (Fig. 15: 5).

Closed forms are relatively rare within phase Va-b: globular jars with everted rims (5\%) (Fig. 15: 14), jars with narrow-banded rims and slight concavities around the rim (5\%) (Fig. 15: 16), flat-topped jars with everted, ledge rims (5\%) (Fig. 15: 13), and a single sherd of a jar with an upright rim (Fig. 15: 15). 
Table 11 Phase Va-b: description of Fig. 15 illustrated sherds.

\begin{tabular}{|c|c|c|c|c|c|c|c|c|c|}
\hline No. & Type & Fabric & Technology & $\begin{array}{l}\text { Exterior Surface } \\
\text { Treatment }\end{array}$ & $\begin{array}{l}\text { Interior } \\
\text { Surface } \\
\text { Treatment }\end{array}$ & Exterior Colour & Interior Colour & Core Colour & $\begin{array}{l}\text { Slip } \\
\text { Colour }\end{array}$ \\
\hline 1 & conical cup with lip & $\mathrm{F} 2 \mathrm{c}$ & wheel thrown & wet smoothed & wet smoothed & pink & pink & pink & \\
\hline 2 & conical cup & $\mathrm{F} 2 \mathrm{c}$ & wheel thrown & wet smoothed & wet smoothed & pink & pink & pink & \\
\hline 3 & conical cup & $\mathrm{F} 2 \mathrm{~b}$ & wheel thrown & wet smoothed & wet smoothed & pink & pink & pink & \\
\hline 4 & conical cup & $\mathrm{F} 2 \mathrm{c}$ & wheel thrown & wet smoothed & wet smoothed & $\begin{array}{l}\text { light reddish } \\
\text { brown }\end{array}$ & $\begin{array}{l}\text { light reddish } \\
\text { brown }\end{array}$ & pink & \\
\hline 5 & simple rim bowl & $\mathrm{F} 2 \mathrm{c}$ & coil & self slip & self slip & very pale brown & very pale brown & very pale brown & \\
\hline 6 & incurved rim bowl & $\mathrm{F} 2 \mathrm{c}$ & $\begin{array}{l}\text { coil and wheel } \\
\text { finish }\end{array}$ & self slip & self slip & pink & pink & pink & \\
\hline 7 & incurved rim bowl & $\mathrm{F} 2 \mathrm{c}$ & coil & $\begin{array}{l}\text { self slip / } \\
\text { burnished }\end{array}$ & self slip & pink & pink & very dark grey & \\
\hline 8 & everted rim bowl & F6 & coil & wet smoothed & wet smoothed & light brown & light brown & very dark grey & \\
\hline 9 & everted rim bowl & $\mathrm{F} 2 \mathrm{c}$ & coil & wet smoothed & wet smoothed & very pale brown & very pale brown & very pale brown & \\
\hline 10 & Beveled Rim Bowl & F1a & mould & rough & wet smoothed & pink & pink & dark grey & \\
\hline 11 & Beveled Rim Bowl & F1a & mould & rough & wet smoothed & pink & pink & dark grey & \\
\hline 12 & Beveled Rim Bowl & F1a & mould & rough & wet smoothed & pink & pink & dark grey & \\
\hline 13 & everted ledge rim jar & F2c & coil & wet smoothed & wet smoothed & pink & pink & pink & \\
\hline 14 & everted rim jar & $\mathrm{F} 5 \mathrm{c}$ & coil & wet smoothed & wet smoothed & $\begin{array}{l}\text { light brownish } \\
\text { grey }\end{array}$ & $\begin{array}{l}\text { light brownish } \\
\text { grey }\end{array}$ & $\begin{array}{l}\text { light brownish } \\
\text { grey }\end{array}$ & \\
\hline 15 & jar w. upright rim & F6 & coil & wet smoothed & wet smoothed & $\begin{array}{l}\text { light brownish } \\
\text { grey }\end{array}$ & $\begin{array}{l}\text { light brownish } \\
\text { grey }\end{array}$ & $\begin{array}{l}\text { light brownish } \\
\text { grey }\end{array}$ & \\
\hline 16 & $\begin{array}{l}\text { angle rim jar with undercut } \\
\text { rim }\end{array}$ & $\mathrm{F} 2 \mathrm{c}$ & coil & wet smoothed & wet smoothed & reddish yellow & reddish yellow & dark grey & \\
\hline 17 & pot stand & F1c & coil & wet smoothed & wet smoothed & pale yellow & pale yellow & pale yellow & \\
\hline 18 & $\begin{array}{l}\text { body sherd with applique } \\
\text { cordon }\end{array}$ & $\mathrm{F} 2 \mathrm{c}$ & coil & self slip & self slip & very pale brown & very pale brown & very pale brown & \\
\hline 19 & $\begin{array}{l}\text { body sherd with rope } \\
\text { cordons }\end{array}$ & F1c & coil & wet smoothed & wet smoothed & grey & grey & grey & \\
\hline 20 & $\begin{array}{l}\text { body sherd with impressed } \\
\text { cordon }\end{array}$ & $\mathrm{F} 2 \mathrm{c}$ & coil & wet smoothed & wet smoothed & reddish yellow & reddish yellow & reddish yellow & \\
\hline 21 & $\begin{array}{l}\text { body sherd with incised } \\
\text { triangles }\end{array}$ & $\mathrm{F} 2 \mathrm{c}$ & coil & burnished & wet smoothed & reddish yellow & reddish yellow & grey & \\
\hline
\end{tabular}




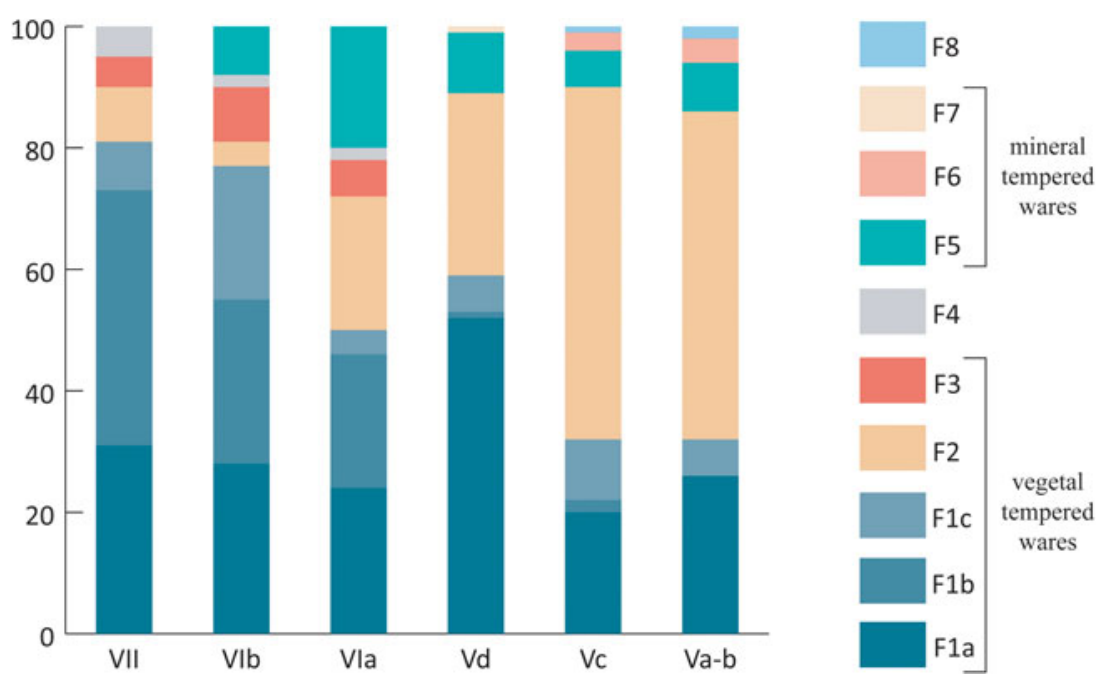

Fig. 16 Rates of fabric group occurrences per phase (percentage of diagnostic sherds).

Further forms not included in the above tallies are a straight cannon spout and a thumb-impressed pot stand (Fig. 15: 17) that has comparisons at Chogha Mish (Delougaz et al. 1996: 47, fig. 8: XVI) and Sheikh Hassan (Boese 1995: 79: fig. 16: g-h, 80: fig. 17: i, 271: fig. 14).

\section{Decoration}

Similar to the previous phase, $10 \%$ of the total diagnostic sherds from this phase have decoration, usually an applique rope cordon (6.3\%) or an impressed rope cordon (3.1\%). Comparative applique rope cordons are noted at, for example, Sheikh Hassan Schicht 10 (Boese 1995: 23: fig. 23: g) and Tell Rubeidheh (McAdam and Mynors 1988: fig. 30: 46; 31: 58). Impressed/incised cordons are noted at Gurga Chiya (Wengrow et al. 2016: 262), Sheikh Hassan (Boese 1995: 201: fig. 13: 1-n), and Tell Rubeidheh (McAdam and Mynors 1988: fig. 32: 78).

One sherd features a horizontal band of incised triangles around the shoulder of the vessel (Fig. 15: 21). Such sherds with incised triangular motifs frequently occur within Late Uruk period ceramic assemblages, with comparative examples from Tell Rubeidheh (McAdam and Mynors 1988: fig. 34: 99) and Godin Tepe (Badler 2002: fig. 17; Rothman and Badler 2011: fig. 4: 47). Another decorated sherd features a double row of rope cordons applied horizontally to the vessel shoulder (Fig. 15: 20). Single rope cordons are well known within contemporary Uruk assemblages, however double-rope cordons are less common. A similar example is depicted from Tell Rubeidheh (McAdam and Mynors 1988: fig. 32: 75).

\section{Fabrics}

F2 continues to dominate phase Va-b at 54\% of total diagnostic sherds (F2a 2\%; F2b 4\%; F2c 48\%) (Fig. 16). F1a, used only for BRBs, represents $26 \%$ of the total diagnostic sherd count, while F1c remains relatively stable at $6 \%$. Mineral tempered fabrics F5c (8\%) and F6 (4\%), as well as F8 $(2 \%)$ remain consistent and overall rare.

\section{Forming Techniques and Surface Treatments}

As in the previous phase, two pottery types show form-specific methods of manufacture: BRBs are all made in an existing mould, and thin-walled, string-cut cups have horizontal rill-marks around the vessel indicative of manufacture on a fast-wheel. Final surface treatment was limited to wet smoothing of the exterior and interior of the vessels, presumably while the vessels were still on the wheel or tournette.

The remainder of diagnostic rim sherds from phase Va-b all show coiling as the principal method of manufacture, with finishing or wet smoothing of the vessel via a wheel or tournette. Surface 


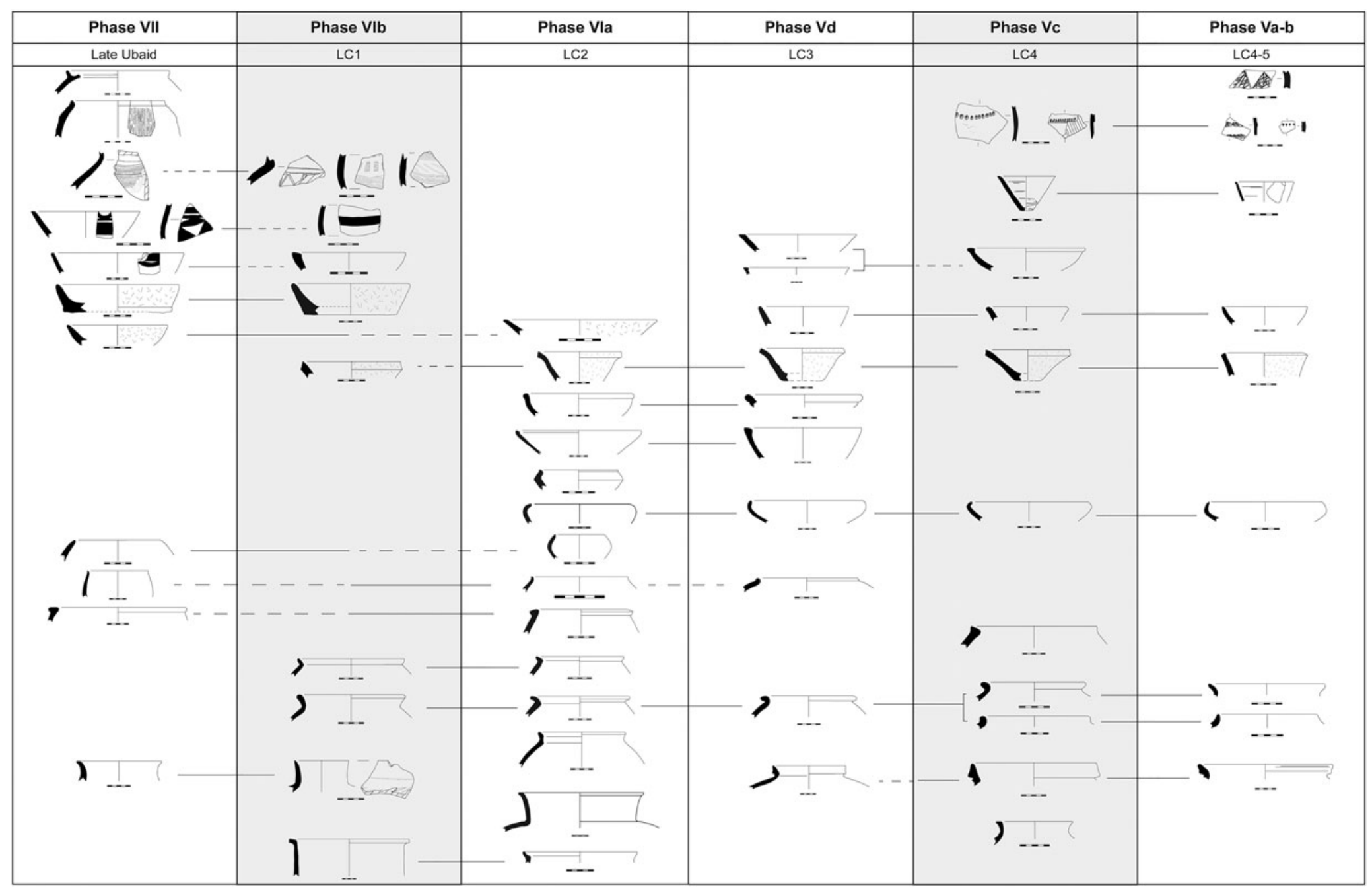

Fig. 17 Chronological development of main ceramic types throughout the LC at Kani Shaie. 


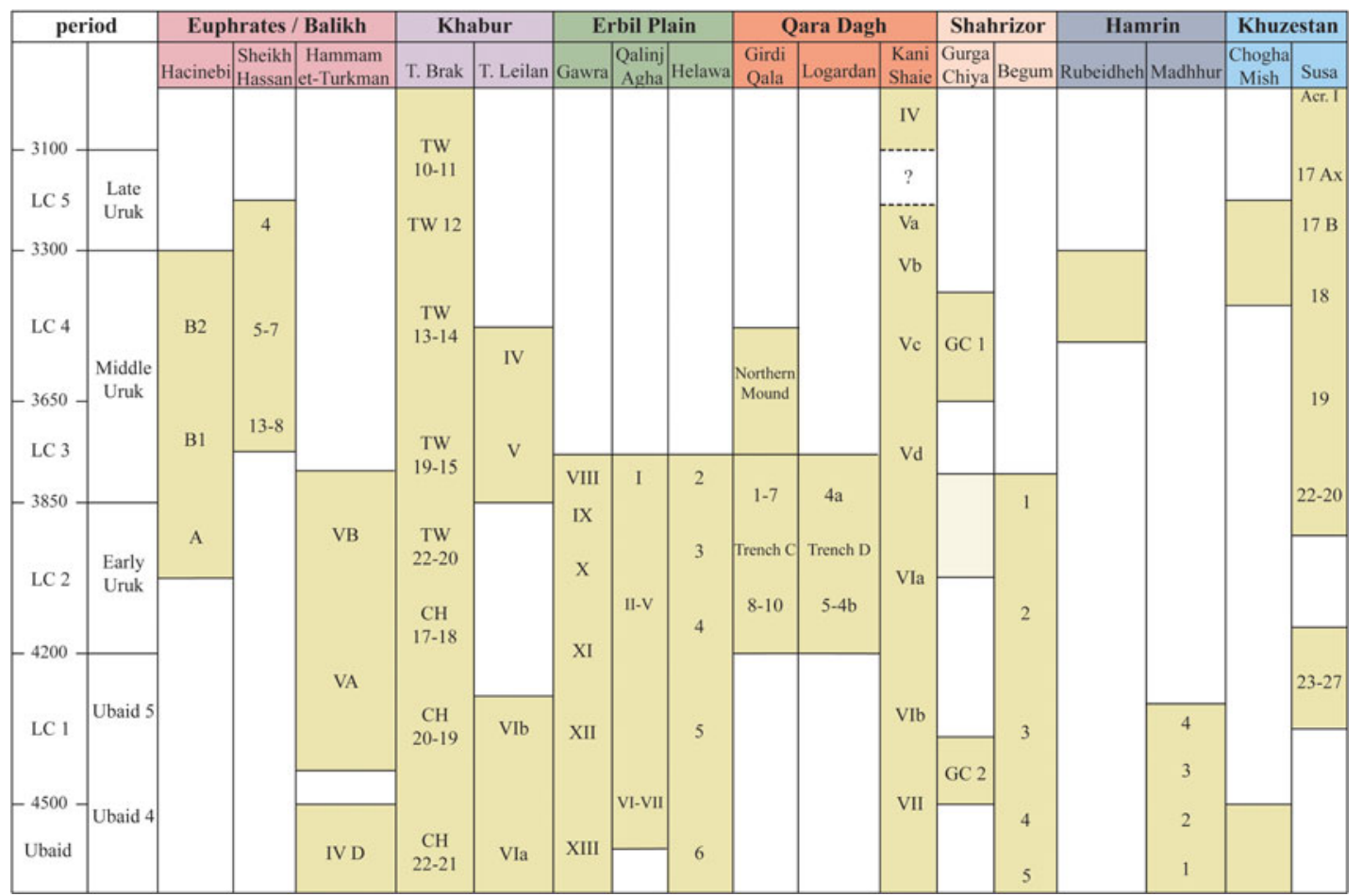

Fig. 18 Chronology chart of major LC sequences from northern Mesopotamia, the Trans-Tigridian Corridor, and Khuzestan.

treatment beyond wet smoothing is rare within this phase, with only one example of a red slip applied to the interior and exterior surface of the banded rim jar with everted rim and flat top, and only one example of burnishing to one of the incurved rim bowls.

\section{Chronology}

In order to begin constructing a regionally relevant ceramic chronology using local sequences, we have avoided as much as possible forcing a priori the Kani Shaie phases into the LC framework that derives mainly from northern Mesopotamian sites. Nevertheless, general similarities in material culture with distant sites allow a preliminary assessment of contemporaneity, pending a robust carbon dated chronology (Fig. 18). ${ }^{14}$

The earliest excavated levels at Kani Shaie, phase VII, can be dated to the late Ubaid/LC1 transition. The best parallels are found in levels said to be Ubaid to LC1 at Tell Helawa, Gurga Chiya, Surezha, and Tell Madhhur. Defining this transition remains a contested issue. At Surezha, carbon dates seem to push the onset of $\mathrm{LCl}$ to the beginning of the fifth millennium B.C.E. (Stein 2018: 42; Stein and Alizadeh 2017: 86). However, the parameters used to assign the ceramic assemblage of Surezha to LC1 differ from those used by other projects. Similarly, at Gurga Chiya, phase GC2 is assigned to the late Ubaid period, even though material from this phase has parallels with assemblages assigned to LC1 at Tell Helawa and Surezha (Carter et al. 2020). We assign Kani Shaie phase VII to the very end of the late Ubaid period, based on the significant presence of painted sherds and Dalma Impressed ware, while the already high percentage $(18.5 \%)$ of MPBs may herald the LC1.

\footnotetext{
${ }^{14}$ Ten samples spanning the LC step trench sequence from Kani Shaie have been submitted for C14 dating, the results of which will be published separately.
} 
Phase VIb, on the other hand, can be reliably assigned to LC1, based on parallels with Gawra XII and assemblages from Tell Helawa and Surezha. In the step trench, this phase occupies almost four meters of deposits that show a remarkable consistency in material culture. This suggests that the LC1 period at Kani Shaie might have lasted quite a long time, which could at least partially explain differences in C14 dates for the same period at sites such as Surezha and Gurga Chiya. ${ }^{15}$ The next phase, VIa, finds its best parallels in LC2 levels at Tell Helawa and Hamoukar.

Phase $\mathrm{Vd}$ is not well represented in the step trench. The material finds good parallels in Gawra VIII, while LC2 ceramic types linger, indicating a date early in LC3. Material from a pit lower on the slope could date slightly later in LC3. Phase Vc can be securely dated to LC4, with an assemblage that has its closest comparanda at nearby Tell Rubeidheh and Gurga Chiya. The earliest level of phase Vc (level 5) still displays continuity from LC3, but not the upper level (4). This phase ends in a destruction that has been observed at different locations in the site, followed by a short period of ephemeral activity in level 3. Finally, phase Va-b still has its best parallels at Tell Rubeidheh and other LC4 sites, but new traits herald LC5 characteristics.

\section{Discussion: The regional context of Kani Shaie}

Admittedly, the limited exposure of the step trench at Kani Shaie means that the dataset presented here is potentially not fully representative. Nonetheless, considering the dearth of complete LC sequences from the Trans-Tigridian region, this assemblage serves as a steppingstone for the construction of a regional ceramic chronology. Beyond a chronological assessment, our ceramic analysis allows a preliminary analysis of technological practices at Kani Shaie. The macroscopically observed fabric typology presented here is preliminary but reveals broad trends. ${ }^{16}$

In general, the ceramic assemblage of Kani Shaie initially exhibits strong parallels to assemblages of sites in the vicinity and gradually becomes more embedded in the trans-regional networks of the LC, while maintaining regionalized traits. Such a phenomenon is not dissimilar to perspectives on LC developments at large (Baldi 2016; Stein 2012). However, this top down approach, which implies that supra-regional processes were enacted upon sites, drawing them ever closer into an overarching Chalcolithic world, needs to take into account micro and medial scales of processes observed at the site level.

To understand social developments pertinent to Kani Shaie, it is useful to envision the various elements within the ceramic assemblage as different units, with their own histories of production embodied in unique chaînes opératoires (Abu Jayyab 2019). Similarities of wares and vessel types between sites can be understood as resulting from the actions of tightly knit groups of producers who form a distinct 'community of practice' constituted through situated learning and apprenticeship (Lave and Wenger 1991). The habits adopted by these communities of practice produce traditions, reflected in ceramic wares, that can be distinguished from those of other groups (Gosselain 1998). If mobility and changing residential patterns form part of a potting community's practices, multiple communities of practice can exist within a given village, while a single community of practice can crosscut multiple villages (Eckert 2008: 2). Through examining how certain traditions and production sequences circulated, we can begin to reconstruct formative historical processes that shaped site assemblages. Such an approach can be used to begin to explain the regional variation of ceramic assemblages and the different degrees of local adoption of foreign vessel types during the LC.

Kani Shaie phase VII produced an eclectic collection of ware groups, with multiple potting traditions existing side-by-side. Worth noting here is the presence of Ubaid-like painted motifs and forms, which suggests that during this phase the inhabitants of the site participated in what has been described as an Ubaid oikumene (Stein and Özbal 2007). Judging by macroscopic

\footnotetext{
${ }^{15}$ At Gurga Chiya, the Late Ubaid/LC1 level (GC2) produced a C14 date ranging between $4530-4340$ cal B.C. (Carter et al. 2020), while the occupation labeled as LC1 at Surezha (phase D-H in Operation 2) produced several C14 dates ranging between 5200-4900 cal B.C. (Stein 2018: 42).
}

\footnotetext{
${ }^{16} \mathrm{M}$. Lewis is developing a detailed fabric typology through petrographic analysis, based on the assemblages from Kani Shaie, Gurga Chiya, and Gird-i Shamlu, as part of his $\mathrm{Ph} . \mathrm{D}$. dissertation.
} 
observations of fabric composition, the Ubaid wares do not seem to have been produced outside of the site or at least outside the valley, however they can certainly be distinguished through elements of their chaînes opératoires. All Ubaid wares recovered were made of finer fabrics. Mineral fabric F4 was exclusively reserved for Ubaid painted and incised wares, while other wares were almost always made with fine chaff and mineral temper. This evidence suggests that during the Ubaid/LC transition at Kani Shaie, Ubaid potting communities of practice were still active at the site, either as persistent remnants of Ubaid traditions, or as part of a still active oikumene of greater Mesopotamian traditions with localized expressions.

Dalma Impressed ware is another distinct tradition that exclusively occurs during phase VII. Dalma Impressed wares occur most commonly in the highlands of the Zagros and only occur in limited numbers in the Trans-Tigridian Piedmont. In the Zagros Mountains, but mainly east of the Chaîne Magistrale, these impressed wares occur together with Dalma Painted ware. However, these do not share many elements in their respective production sequences (Henrickson and Vitali 1987; Sorkhani and Eslami 2018). A common explanation for these differences in distribution and production is that Dalma Impressed ware was part of the repertoire of mobile communities that frequently moved between the high Zagros valleys and the piedmont during the Middle Chalcolithic (Henrickson 1986, 1989; Tonoike 2009, 2012). The majority of the Dalma Impressed wares from Kani Shaie seem to have been produced locally, with the fabrics predominantly made from a clay containing limestone with vegetal temper inclusions. One sample made from a micarich clay could have been brought in from outside the region.

Phase VII also produced MPBs, which is a mass-produced bowl type that became common during LC1-2. However, it would be premature to tie this phase into a typo-chronological scheme that was developed from assemblages west of the Tigris (Schwartz 2001). Alternatively, we suggest that phase VII shares elements that have been traditionally considered Ubaid with some elements that belong in LC1. Without a clear absolute chronology and with limited knowledge of region-specific sequences, we cannot say for certain if the concept of MPBs arrived from the areas west of the Tigris, were an early indigenous practice, or resulted from a more complicated process. What we can say is that the phase VII assemblage of Kani Shaie resulted from the overlap of distinct traditions conceived in different communities of practice - coming from different directions and coalescing at the site, which is a persistent pattern throughout the entire Kani Shaie sequence.

The next phase, VIb, shows continuity with phase VII in terms of potting practices, but with the addition of new elements, such as grey ware cooking pots that appear for the first time. These pots are unique in terms of their chaîne opératoire, with primarily calcite temper (fabric F5) and micaceous clays. Moreover, the overall shape of these vessels, their surface treatments (smoothing and burnishing), and their firing in a reduced atmosphere distinguish them from other wares at the site. These vessels may represent a change in diet, as they are best equipped to cook gruels and soups, a practice not evident in the previous phase.

Another major shift is seen in decoration preference, with incised wares overtaking painted wares in phase VIb. Changes in potting traditions and aesthetic preferences had clearly shifted, replaced by potting traditions of new communities of practice. These potting communities became much more limited in their interaction networks, restricted mainly to the area between the Upper Zab and Diyala/Sirwan rivers and the Tigris and Zagros Mountains. MPBs are the only ceramic type with a wider distribution, but even within this broad category the MPBs at Kani Shaie belong to a variant ("Wide Flower Pots") that mainly occurs in the eastern part of northern Mesopotamia. At the same time, disappearance of painted wares, emergence of mass-produced wares, appearance of specialized cooking wares, and increased regionalism are developments that occur throughout southwest Asia in LC1 (Abu Jayyab 2019; Baldi 2016; Stein 2012).

After a period of intra-regional entrenchment, phase VIa (LC2) shows a reemergence of interregional connections. This period is marked by an increase in BRBs, perhaps reflecting new contacts with southern Mesopotamia, together with a decrease of earlier MPBs. While grey cooking pots remain numerous, a number of vessels usually associated with assemblages of the Syrian Jazira, such as internally beveled rim bowls, internally hollowed rim jars, and carinated black burnished wares, become part of the repertoire of the potters at Kani Shaie. Nevertheless, 
while there is a reemergence of external influences from Mesopotamia, the overall potting traditions at Kani Shaie mostly show continuity from the previous phase while carefully borrowing elements that were circulating through interregional networks.

This trend of gradual adoption of widespread ceramic wares continues into phase $\mathrm{Vd}$. The dominance of vegetal temper together with the lack of surface treatment other than wet smoothing fits well within the broader chaff-faced ware oikumene, demonstrative of the relative homogenisation of ceramic regions across northern Mesopotamia and the northern Zagros (Baldi 2016; Marro 2010). However, the phase Vd ceramic repertoire from Kani Shaie retains a distinctly regionalised flavour shared with the LC2-3 strata at Girdi Resh, Girdi Shamlu, and Gurga Chiya in the Shahrizor. In addition to the disappearance of several ceramic shapes, the most noteworthy change from phase VIa to $\mathrm{Vd}$ is the remarkable increase of BRBs from $3.5 \%$ to over $50 \%$ of the diagnostic assemblage, alongside the virtual disappearance of earlier MPBs and the appearance in their place of simple-rimmed bowls.

Phase Vd features a relatively limited range of forms, which are long-lived within the archaeological strata (mainly BRBs and simple everted rim globular jars). Characteristic forms of this phase are few, yet chronologically sensitive. Thin-walled bowls with a beveled rim closely resemble the coarse BRB, but they differ markedly in their finesse and are much less common. The assemblage of this phase is somewhat difficult to place temporally as it lies stratigraphically between the heavily studied LC1-2 horizon and the well-known ceramic forms of the later LC and the Uruk Phenomenon. LC3, in contrast, is an understudied period in the region due to widespread site abandonment (e.g., Helawa: Peyronel and Vacca 2015). The as yet small, but important, phase $\mathrm{Vd}$ assemblage from Kani Shaie provides an anchor point that can elucidate the transition from the early to the later LC.

Phase Vc initially shows strong continuity from Vd but sees the rapid introduction of a completely new tradition. BRBs remain the most dominant open form, as well as V-shaped bowls with simple rims and globular jars with plain everted rims. Fine, thin-walled bowls with beveled rims continue yet are found exclusively within the earliest strata of this phase. Similarly, club-headed bowls and flat-topped, square profile bowls that are traditionally associated with earlier LC2 -3 strata indicate a prolonged production and conservatism in ceramic trends. On the other hand, several ceramic forms that herald the beginnings of a typical southern "Uruk" ceramic repertoire appear and rapidly increase in importance in the upper level of phase Vc. These include fine conical cups, nose-lugged jars, jars with incised or applique 'rope cordons', spouted jars (mainly cannon spouts), and incurved-rim bowls, which closely resemble assemblages from the Hamrin sites. Interestingly, some of the conical cups feature scraped bottoms and bases rather than the slightly later string-cut bases, reminiscent of earlier LC ceramic traditions associated especially with MPBs. This suggests that local potters initially maintained their traditional practice of producing a flat base through scraping before adopting the new technology of production on a fast wheel, resulting in the string-cut bases that commonly define this type.

Macroscopic analysis of production techniques of the vessels mainly indicates continuity with earlier phases. Most vessels from phase Vc show evidence that the vessel rough-out was formed using a tournette or other rotary device, with coils added to form the upper body, and a final coil added for the rim. The continued lack of surface treatment within this phase is a persistent regionalised expression. This is particularly interesting given the contemporary assemblage at Gurga Chiya, where slipping and burnishing is widespread (Wengrow et al. 2016: 262-263). 'Classic' Uruk wares are present at Kani Shaie, mainly out of context at present, but are for as yet unknown reasons less attested within the step trench assemblage (Fig. 14; Tomé et al. 2016: fig. 4). Furthermore, the frequently asserted dichotomy between 'local' LC assemblages with vegetal temper versus 'Uruk' assemblages with mineral temper cannot be maintained at Kani Shaie, as has already been observed at other sites in the region (Carter et al. 2020; Vallet et al. 2017: 75).

The assemblage of final phase Va-b is very similar to that of the upper level of Vc. Surface treatment beyond wet-smoothing is virtually absent, and manufacturing of the vessels is still dominated by a mould-made base and lower body, with coils added to form the remainder of the vessel. BRBs still feature as the dominant form of phase $\mathrm{Va}-\mathrm{b}$, while conical cups, globular jars 
with simple everted rims and incurved-rim bowls form the majority of the assemblage. Despite this continuity, phase Va-b sees the introduction of LC5 ceramic characteristics. Dating LC5/Late Uruk assemblages from the region is particularly difficult given the complete absence of securely radiocarbon dated strata belonging to this period. Nevertheless, we tentatively ascribe an early LC5 date to phase Va-b, based on a number of observations. BRBs from this phase are somewhat taller with a more upright stance and a pinker fabric than those of earlier LC phases, which matches observations by Helwing (2014) and compares well with the BRBs of the EBA in the central Zagros and also observed in phase IV at Kani Shaie. Additionally, increased quantities of conical cups both with and without pouring lip in this phase match observations from the Late Uruk strata of the Uruk Mound at Abu Salabikh (Pollock 1987: 127). The presence of specific decorative elements, particularly incised cross-hatched triangles, is also a later development (Wright 2013: 68). While drooping spouts are commonly used as a definitive marker of LC5 ceramic assemblages, it is interesting to note that these are at present virtually absent in the wider south-eastern Kurdistan region of Iraq and the Hamrin. Also currently lacking from the admittedly small phase Va-b assemblage are strap-handled jars, twisted handles, sharply carinated bowls, trays, and lids. The excavators of Tell Rubeidheh expressed similar difficulties with dating their ceramic assemblages, even highlighting potential Jemdet Nasr or EBA types (Crawford 1988: 136). A possible explanation is a higher degree of conservatism in ceramic traditions in the TransTigridian region, resulting in an assemblage that does not match contemporary developments further west.

\section{Conclusion}

Despite current debate that increasingly recognizes the regionalised nature of the Ubaid (Carter and Philip 2010) and early LC (Marro 2012), the long-term developments of the LC are frequently framed within a cultural evolutionary model that culminates in the dominance of southern Mesopotamia by the end of the fourth millennium B.C.E. Rather than a top-down approach that emphasizes general similarities spread through interregional networks, stability and gradual change in potting traditions at a single site can be analyzed as the reflection of a historical process of creation and dissolution of cultural boundaries. In this discussion of the ceramic development at Kani Shaie, we have taken a bottom-up approach that traces overlapping actions of communities of practice. The significance of the Bazian passage as a conduit for movement between Mesopotamia and the Zagros, in addition to Kani Shaie's access to pasture, farmland, and fresh water sources, should not be downplayed. This geo-historical condition shaped the site, as evidenced by the longevity of occupation. Kani Shaie was a central focus of settlement within the Bazian Basin for millennia, not only during times of intense mobility and interregional interactions, but also in periods of reduced external interaction (e.g. phase VIb).

While changes in potting traditions at Kani Shaie can be interpreted as local choices, it is striking that the community opted to orientate themselves overwhelmingly toward Mesopotamia. This is especially surprising considering that the Bazian Basin provides an environment that has much more in common with the Zagros intermontane valleys than the Mesopotamian lowlands. At present, not a single sherd shows connections with the numerous Chalcolithic Zagros painted traditions that were in use even within a few days travel. This contrasts sharply with the early EBA period at Kani Shaie (phase IV) when the community closely engaged with Zagros potting traditions (Renette 2018).

Overall, the Kani Shaie LC ceramic sequence parallels developments that have been observed throughout northern Mesopotamia, while revealing distinctly regional traditions. Assemblages of every period find their best parallels within the Trans-Tigridian region and especially within the southern Kurdistan region of Iraq. While the earlier LC of Kani Shaie shares many elements with sites from the Upper Zab to the Diyala/Sirwan River, the later LC is more narrowly focused on the Adhaim-Diyala/Sirwan River drainage system south of the Lower Zab, encompassing presentday Sulaymaniyah, Kirkuk, and the Hamrin Basin. Additional connections along the western Zagros flanks to Khuzestan (especially Chogha Mish) further support a change in orientation southward. This regionalism of late LC pottery assemblages has been highlighted previously 
(Trentin 1991: 8) but remains poorly documented and underappreciated. Regionalised ceramic assemblages are not commonly associated with Uruk period archaeology, yet ethnographic examples show just such a scenario, with potters producing similar, although somewhat different, versions of what is traditionally grouped together as a coherent assemblage (Dietler and Herbich 1994: 463). Arguably, at Kani Shaie the local potters were tasked with producing vessels within the broader remit of the Uruk phenomenon, whilst maintaining certain specific ceramic forms that more closely served the immediate needs of the local community.

\section{Bibliography}

Abu al-Soof, B. 1985. Uruk Pottery: Origins and Distribution. Baghdad: Ministry of Culture and Information, State Organization of Antiquities and Heritage.

Abu Jayyab, K. 2012. "A Ceramic Chronology from Tell Hamoukar's Southern Extension" in C. Marro, ed. After the Ubaid: Interpreting Change from the Caucasus to Mesopotamia at the Dawn of Urban Civilization (4500-3500 BC). Paris: De Boccard, pp. 87-128.

2019. Nomads in Late Chalcolithic Northern Mesopotamia: Mobility and Social Change in the $5^{\text {th }}$ and $4^{\text {th }}$ Millennia BC. Unpublished Ph.D. dissertation, University of Toronto.

Akkermans, P. M. M. G. 1988. "The Period V Pottery" in M. van Loon, ed. Hammam et-Turkman I. Report on the University of Amsterdam's 1981-84 Excavations in Syria. Istanbul: Nederlands HistorischArcheologisch Instituut, pp. 287-349.

Akkermans, P. M. M. G. and G. M. Schwartz. 2003. The Archaeology of Syria: From complex hunter-gatherers to early urban societies (c. 16,000-300 BC). Cambridge: Cambridge University Press.

Al Quntar, S. and K. Abu Jayyab. 2014. "The Political Economy of the Upper Khabur in the Late Chalcolithic 1-2: Ceramic mass production, standardization and specialisation" in A. McMahon and H. Crawford, eds. Preludes to Urbanism: The Late Chalcolithic of Mesopotamia. Cambridge: McDonald Institute for Archaeological Research, pp. 89-108.

Alden, J. R. 1988. "Ceramic ring scrapers: An Uruk period pottery production tool”. Paléorient 14: 143-150.

Alden, J. R. and L. Minc. 2016. "Itinerant potters and the transmission of ceramic technologies and styles during the Proto-Elamite period in Iran". Journal of Archaeological Science: Reports 7: 863-876.

Algaze, G. 1993. The Uruk World System. The Dynamics of Expansion of Early Mesopotamian Civilization. Chicago: University of Chicago Press.

2008. Ancient Mesopotamia at the Dawn of Civilization. The Evolution of an Urban Landscape. Chicago: University of Chicago Press.

Alizadeh, A. 2009. Chogha Mish II: The Development of a Prehistoric Regional Center in Lowland Susiana, Southwestern Iran. Final Report on the Last Six Seasons of Excavations, 1972-1978. Oriental Institute Publications 130. Chicago: The Oriental Institute.

Badler, V. R. 2002. "A Chronology of Uruk Artefacts from Godin Tepe in Central Western Iran and Its Implications for the Interrelationships Between Local and Foreign Cultures" in J. N. Postgate, ed. Artefacts of Complexity: Tracking the Uruk in the Near East. Iraq Archaeological Reports 5. Warminster: British School of Archaeology in Iraq, pp. 79-110.

Baldi, J. S. 2012. "Coba Bowls, Mass Production and Social Change in Post-Ubaid Times" in C. Marro, ed. After the Ubaid: Interpreting Change from the Caucasus to Mesopotamia at the Dawn of Urban Civilization (4500-3500 BC). Paris: De Boccard, pp. 393-416.

2016. "Regionalized Patterns and Paths to Complexity: Reflections on Ceramic Provinces and Organizational Modalities in $6^{\text {th }}$ and $4^{\text {th }}$ Millennia Northern Mesopotamia" in M. Iamoni, ed. Trajectories of Complexity: Socio-economic Dynamics in Upper Mesopotamia in the Neolithic and Chalcolithic Periods. Studia Chaburensia 6. Wiesbaden: Harrassowitz, pp. 117-138.

2017. "Chalcolithic Ceramics from Logardan Trenches D and E: Morpho-stylistic features and regional parallels" in R. Vallet, ed. Report on the Third Season of Excavations at Girdi Qala and Logardan. Paris: Institut Français du Proche-Orient, pp. 57-66.

Baldi, J. S., M. Iamoni, L. Peyronel and P. Sconzo, eds. in press. Societies in Contact: Late Chalcolithic and Uruk Mesopotamian Communities at the Dawn of the Urban World. Subartu. Turnhout: Brepols.

Ball, W., ed. 2003. Ancient Settlement in the Zammar Region. BAR International Series 1096. Oxford: Archaeopress.

Benati, G. 2018. "The Construction of Large-Scale Networks in Late Chalcolithic Mesopotamia: Emergent Political Institutions and Their Strategies" in D. Domenici and N. Marchetti, eds. Urbanized Landscapes in Early Syro-Mesopotamia and Prehispanic Mesoamerica: Papers of a Cross-Cultural Seminar held in Honor of Robert McCormick Adams. Wiesbaden: Harrassowitz, pp. 103-143.

Boese, J. 1995. Ausgrabungen in Tell Sheikh Hassan I: Vorlaufige Berichte über die Grabungskampagnen 1984-1990 und 1992-1994. Schriften zür vorderasiatischen Archäologie 5. Saarbrücken: Saarbrücker Druckerei. 
Braidwood, R. J. and B. Howe. 1960. Prehistoric Investigations in Iraqi Kurdistan. Studies in Ancient Oriental Civilization 31. Chicago: University of Chicago Press.

Butterlin, P. 2003. Les temps proto-urbains de Mésopotamie. Contacts et acculturation à l'époque d'Uruk au Moyen-Orient. Paris: CNRS Éditions.

Butterlin, P., ed. 2009. A propos de Tepe Gawra, le monde proto-urbain de Mésopotamie. Subartu XXIII, Turnhout: Brepols.

Butterlin, P. 2018. Architecture et société au Proche-Orient ancien: les bâtisseurs de mémoire en Mésopotamie (7000-3000 av. J.-C.). Paris: Éditions Picard.

Carter, R. A. and G. Philip, eds. 2010. Beyond the Ubaid: Transformation and Integration in the Late Prehistoric Societies of the Middle East. Studies in Ancient Oriental Civilization 63. Chicago: The Oriental Institute.

Carter, R., D. Wengrow, M. Shepperson, K. Roberts, M. P. Lewis, A. Marsh, L. Gonzales-Carretero, H. Sosnowska, A. D'Amico, W. Sagan and K. Lockyear. 2020. "The later prehistory of the Shahrizor Plain, Iraqi Kurdistan: Further investigations at Gurga Chiya and Tepe Marani”. Iraq 82: 41-71.

Catanzariti, A., T. Tanaka and A. Maskevich. 2020. "Ban Qala: A Late Chalcolithic Site in the Qara Dagh Valley of Iraqi Kurdistan" in A. Otto, M. Herles and K. Kaniuth, eds. Proceedings of the $11^{\text {th }}$ International Congress on the Archaeology of the Ancient Near East, Vol. 2 Field Reports, Wiesbaden: Harrassowitz, pp. 43-54.

Crawford, H. 1988. "Conclusion” in R.G. Killick, ed. Excavations at Tell Rubeidheh. Warminster: British School of Archaeology in Iraq, pp. 136-138.

Dahl, J., C. A. Petrie and D. T. Potts. 2013. "Chronological Parameters of the Earliest Writing System in Iran" in C. A. Petrie, ed. Ancient Iran and its Neighbours: Local Developments and Long-range Interactions in the Fourth Millennium BC. British Institute of Persian Studies Archaeological Monograph Series III. Oxford: Oxbow, pp. 353-379.

Delougaz, P., H. J. Kantor and A. Alizadeh. 1996. Chogha Mish Volume I: The First Five Seasons of Excavations, 1961-1971. Oriental Institute Publications 101. Chicago: The Oriental Institute.

Dietler, M. and I. Herbich. 1994. "Ceramics and Ethnic Identity: Ethnoarchaeological Observations on the Distribution of Pottery Styles and the Relationship between the Social Contexts of Production and Consumption" in D. Binder and F. Audouze, eds. Terre Cuite et Société: La Céramique, Document Technique, Économique, Culturel. XIVe Rencontre Internationale d'Archéologie et d'Histoire d'Antibes. Juan-les-Pins: Éditions APDCA, pp. 459-472.

Eckert, S. L. 2008. Pottery and Practice: The Expression of Identity at Pottery Mound and Hummingbird Pueblo. Albuquerque: University of New Mexico Press.

Felli, C. 2003. "Developing Complexity: Early to Middle Fourth-millennium Investigations: The Northern Middle Uruk Period" in R. Matthews, ed. Excavations at Tell Brak, vol. 4: Exploring an Upper Mesopotamian Regional Centre, 1994-1996. Cambridge: McDonald Institute, pp. 53-95.

Frangipane, M. 2018. "Different Trajectories in State Formation in Greater Mesopotamia: A View from Arslantepe (Turkey)". Journal of Archaeological Research 26: 3-63.

Gosselain, O. 1998. "Social and Technical Identity in a Clay Crystal Ball" in M. Stark, ed. The Archaeology of Social Boundaries. Washington DC: Smithsonian Institute, pp. 78-106.

Gut, R. V. 1995. Das Prähistorische Ninive. Zur relative Chronologie der frühen Perioden Nordmesopotamiens. Baghdader Forschungen 19. Mainz: Philipp von Zabern.

Hamlin, C. 1975. "Dalma Tepe". Iran 13: 111-128.

Helwing, B. 2014. "Bevelled Rim Bowls" in M. Lebeau, ed. ARCANE Interregional: Ceramics. ARCANE Interregional 1. Turnhout: Brepols, pp. 31-39.

Henrickson, E. F. 1985. "An Updated Chronology of the Early and Middle Chalcolithic of the Central Zagros". Iran 23: 63-108.

— 1986. "Ceramic Evidence for Cultural Interaction between Chalcolithic Mesopotamia and Western Iran" in W. D. Kingery, ed. Technology and Style. Ceramics and Civilization II. Columbus: American Ceramic Society, pp. 87-132.

1989. "Pottery Evidence for Cultural Interaction between the "Ubaid tradition and the Central Zagros Highlands, Western Iran" in E. F. Henrickson and I. Thuesen, eds. Upon this Foundation The 'Ubaid Reconsidered. Carsten Niebuhr Institute Publications 10. Copenhagen: Tusculanum Press, pp. 368-403.

1994. "The outer limits: settlement and economic strategies in the central Zagros highlands during the Uruk era" in G. Stein and M. S. Rothman, eds. Chiefdoms and Early States in the Near East: The Organizational Dynamics of Complexity. Madison: Prehistory Press, pp. 85-102.

Henrickson, E. F. and V. Vitali. 1987. "The Dalma Tradition: Prehistoric Inter-Regional Cultural Integration in Highland Western Iran". Paléorient 13: 37-45.

Hijara, I. 1973. "Excavations at Qalinj Agha (Erbil), $4^{\text {th }}$ Season”. Sumer 29: 13-35. 
1976. "Excavations at Shahrazur Plain, Tell Kurdrsh". Sumer 32: 59-80.

Jasim, S. A. 1985. The Ubaid Period in Iraq. Recent Excavations in the Hamrin Region. BAR International Series 267. Oxford: Archaeopress.

Kepinski, C. 2011. "New Evidence from Grai Resh, Northern Iraq - the 2001 and 2002 Seasons. A Pre-Uruk Expansion Site from the Late Chalcolithic Period". Zeitschrift für Orient-Archäologie 4: 47-85.

Killick, R. G., ed. 1988. Tell Rubeidheh. An Uruk village in the Jebel Hamrin. Iraq Archaeological Reports 2. Hamrin Salvage Project Report 7. Warminster: Aris \& Phillips.

Kopanias, K., C. Beuger, T. Carter, S. Fox, A. Hadjikoumis, G. Kourtessi-Philippakis, A. Livarda and J. MacGinnis. 2013. "The Tell Nader and Tell Baqrta Project in the Kurdistan Region of Iraq: Preliminary Report of the 2011 Season”. SUBARTU (Archaeological Journal of the Kurdistan Region of Iraq) 6-7: 23-57.

Lave, J. and E. Wenger. 1991. Situated Learning: Legitimate Peripheral Participation. Cambridge: Cambridge University Press.

Le Brun, A. 1971. "Recherches stratigaphiques à 1'Acropole de Suse, 1969-1971”. Cahiers de la Délégation archéologiques française en Iran 1: 163-216.

1978. "Suse, chantier Acropole 1". Paléorient 4: 177-192.

Levine, L. D. and T. C. Young. 1987. "A Summary of the Ceramic Assemblages of the Central Western Zagros from the Middle Neolithic to the Late Third Millennium B.C." in J.-L. Huot, ed. Préhistoire de la Mésopotamie: La Mésopotamie préhistorique et l'exploration récente du djebel Hamrin. Paris: Éditions du CNRS, pp. 15-53.

Marro, C. 2010. "Where did Late Chalcolithic Chaff-Faced Ware Originate? Cultural Dynamics in Anatolia and Transcaucasia at the Dawn of Urban Civilization (ca. 4500-3500 BC)". Paléorient 36: 35-55.

Marro, C., ed. 2012. After the Ubaid: Interpreting change from the Caucasus to Mesopotamia at the dawn of urban civilization (4500-3500 BC). Papers from the Post-Ubaid Horizon in the Fertile Crescent and Beyond, International Workshop held at Fosseuse, $29^{\text {th }}$ June- $1^{\text {st }}$ July 2009. Varia Anatolica. Paris: De Boccard.

Matthews, R. 2003. "Traces of Early Complexity. Late Fifth to Early Fourth-millennium Investigations: The Early Northern Uruk Period" in R. Matthews, ed. Excavations at Tell Brak, Vol. 4: Exploring an Upper Mesopotamian Regional Centre, 1994-1996. Cambridge: McDonald Institute Monographs, pp. $25-51$.

2013. "The power of writing: administrative activity at Godin Tepe, Central Zagros, in the later fourth millennium BC" in C.A. Petrie, ed. Ancient Iran and Its Neighbours: Local Developments and LongRange Interactions in the Fourth Millennium BC. British Institute of Persian Studies, Archaeological Monographs Series III. Oxford: Oxbow, pp. 337-352.

Matthews, R. and H. Fazeli. 2004. "Copper and Complexity: Iran and Mesopotamia in the Fourth Millennium B.C." Iran 42: 61-75.

McAdam, E. and H. S. Mynors. 1988. "Tell Rubeidheh: Pottery from the Uruk mound” in R. G. Killick, ed. Tell Rubeidheh: An Uruk village in the Jebel Hamrin. Hamrin Salvage Project Report 7. Warminster: British School of Archaeology in Iraq, pp. 39-76.

McMahon, A. 2020. "Early Urbanism in Northern Mesopotamia”. Journal of Archaeological Research 28: 289337.

Miller, A. 1981. "Straw Tempered Ware” in H. T. Wright, ed. An Early Town on the Deh Luran Plain: Excavation at Tepe Farukhabad. Memoirs of the Museum of Anthropology 13. Ann Arbor: University of Michigan, pp. 126-130.

Molist, M., Z. Bradosty, A. Breu, J. Sisa, R. Alcántara, W. Cruells, C. Douché, P. Mylona, R. Arnaiz, M. Saña, A. Zebari and A. Gómez-Bach. 2019. "New data on the $4^{\text {th }}-3^{\text {rd }}$ millennia in Northern Mesopotamia: The ancient occupations at Gird Lashkir in their archaeological contexts". Paléorient 45(2): 191-206.

Moon, J. and M. Roaf. 1984. "The Pottery from Tell Madhhur”. Sumer 43: 128-158.

Mühl, S. 2013. Siedlungsgeschichte im mittleren Osttigrisgebiet: Vom Neolithikum bis in die neuassyrische Zeit. Abhandlungen der Deutschen Orient-Gesellschaft Band 28. Wiesbaden: Harrassowitz.

Mühl, S. and O. Nieuwenhuyse. 2016. "Halaf and Ubaid period settlement: A view from the Central Zagros Piedmont" in M. Iamoni, ed. Trajectories of Complexity: Socio-economic Dynamics in Upper Mesopotamia in the Neolithic and Chalcolithic Periods. Studia Chaburensia 6. Wiesbaden: Harrassowitz, pp. 27-56.

Nannucci, S. 2012. "La ceramica dei livelli Uruk di Tell Hassan, Hamrin” in S. Mazzoni, ed. Studi di Archeologia del Vicino Oriente. Scritti degli allievi fiorentini per Paolo Emilio Pecorella. Firenze: Firenze University Press, pp. 39-65.

Nieuwenhuyse, O., T. Odaka, A. Kaneda, S. Mühl, K. Rasheed and M. Altaweel. 2016. "Revisiting Tell Begum: A prehistoric site in the Shahrizor Plain, Iraqi Kurdistan". Iraq 78: 103-135.

Nissen, H. J. 1970. “Grabung in den Quadraten K/L XII in Uruk-Warka”. Baghdader Mitteilungen 5: 101-191. 
2001. "Cultural and Political Networks in the Ancient Near East during the Fourth and Third Millennia B. C." in M. S. Rothman, ed., Uruk Mesopotamia and Its Neighbors; Cross-Cultural Interactions in the Era of State Formation. Santa Fe: School of American Research Press, pp. 149-179.

Numoto, H. 1998. "Late Uruk and the Transitional Ninevite 5 Pottery from Tell Thalathat No. 5". Al-Rafidan 19: 53-73.

Oates, J. 2012. "The Terminal Ubaid (LC 1) Level at Tell Brak" in C. Marro, ed. After the Ubaid: Interpreting Change from the Caucasus to Mesopotamia at the Dawn of Urban Civilization (4500-3500 BC). Paris: De Boccard, pp. 65-86.

Oates, J., A. McMahon, P. Karsgaard, S. al-Quntar and J. Ur. 2007. "Early Mesopotamian Urbanism: A View from the North." Antiquity 81(313): 585-600.

Pearce, J. 2000. "The Late Chalcolithic Sequence at Hacinebi Tepe, Turkey" in C. Marro and H. Hauptmann, eds. Chronologies des Pays du Caucase et de l'Euphrate aux IVe-IIIe Millénaires. Paris: Institute français d'Études Anatoliennes d'Istanbul, pp. 115-144.

Petrie, C. A., ed. 2013. Ancient Iran and Its Neighbours: Local Developments and Long-Range Interactions in the Fourth Millennium BC. British Institute of Persian Studies, Archaeological Monographs Series III. Oxford: Oxbow.

Petrie, C. A. 2014. "Iran and Uruk Mesopotamia: Chronologies and Connections in the Fourth Millennium BC" in A. McMahon and H. Crawford, eds. Preludes to Urbanism: The Late Chalcolithic of Mesopotamia. Cambridge: McDonald Institute for Archaeological Research, pp. 137-156.

Peyronel, L. and A. Vacca. 2015. "Northern Ubaid and Late Chalcolithic 1-3 Periods in the Erbil Plain. New Insights from Recent Researches at Helawa, Iraqi Kurdistan”. Origini 37: 89-127.

Peyronel, L., A. Vacca and G. Zenoni. 2016. "Helawa: A New Northern Ubaid/Late Chalcolithic Site in the Erbil Plain" in K. Kopanias and J. MacGinnis, eds. The Archaeology of the Kurdistan Region of Iraq and Adjacent Regions. Oxford: Archaeopress, pp. 309-322.

Pfälzner, P., H. A. Qasim, P. Sconzo and I. Puljiz. 2017. "Report on the First Season of German-Kurdish Excavations at Muqable in 2015”. Zeitschrift für Orient-Archäologie 10: 44-96.

Pollock, S. 1987. "Abu Salabikh, the Uruk Mound 1985-86". Iraq 49: 121-141.

Porter, A. 2012. Mobile Pastoralism and the Formation of Near Eastern Civilizations: Weaving Together Society. Cambridge: Cambridge University Press.

Postgate, J. N., ed. 2002. Artefacts of Complexity: Tracking the Uruk in the Near East. Iraq Archaeological Reports 5. Warminster: British School of Archaeology in Iraq.

Potts, D. T., K. Radner, A. Squitieri, A. Ameen, J. Rohde, P. Yawar, J.-J. Herr, H. Salih, F. Petchey, A. Hogg, B. Gratuze, K. R. Raheem and H. B. Potts. 2019. "Gird-i Rostam 2018: Preliminary Report on the First Season of Excavations by the Joint Kurdish-German-American Team". Journal of the Ancient Near Eastern Society 'Ex Oriente Lux' 47: 91-127.

Reichel, C. 2008. "Hamoukar”. Oriental Institute 2007-2008 Annual Report: 76-82.

— 2011. "Hamoukar 2005-2010: Revisiting the Origins of Urbanism in Syria". Oriental Institute News \& Notes 211: 3-9.

Renette, S. 2016. "Traders of the Mountains. The Early Bronze Age in Iraqi Kurdistan”. Expedition 58(1): 16-23.

2018. Along the Mountain Passes: Tracing Indigenous Developments of Social Complexity in the Zagros Region during the Early Bronze Age (ca. 3500-2000 BCE). Unpublished Ph.D. dissertation, University of Pennsylvania.

Renette, S. \& S. Mohammadi Ghasrian. 2020. "The central and northern Zagros during the Late Chalcolithic: An updated ceramic chronology based on recent fieldwork results in western Iran", Paléorient 46(1): 109-132.

Roaf, M. 1989. "'Ubaid Social Organization and Social Activities as seen from Tell Madhhur" in E. F. Henrickson and I. Thuesen, eds. Upon this Foundation - The 'Ubaid Reconsidered. Carsten Niebuhr Institute Publications 10. Copenhagen: Museum Tusculanum Press, pp. 91-147.

Roaf, M. and R. Killick. 1987. "A Mysterious Affair of Styles: The Ninevite 5 Pottery of Northern Mesopotamia". Iraq 49: 199-230.

Rothman, M. S., ed. 2001. Uruk Mesopotamia and Its Neighbors. Cross-Cultural Interactions in the Era of State Formation. Santa Fe: School of American Research Press.

Rothman, M. S. 2002. Tepe Gawra: The Evolution of a Small Prehistoric Center in Northern Iraq. Philadelphia: University of Pennsylvania Museum Press.

2013. "Interpreting the role of Godin Tepe in the 'Uruk' Expansion" in C. A. Petrie, ed. Ancient Iran and its Neighbours: Local Developments and Long-range Interactions in the Fourth Millennium BC. British Institute of Persian Studies Archaeological Monograph Series III. Oxford: Oxbow, pp. 75-91.

Rothman, M. S. and V. Badler. 2011. "Contact and Development in Godin Period VI" in H. Gopnik and M. S. Rothman, eds. On the High Road: The History of Godin Tepe, Iran. Toronto: Mazda Press, pp. 67-137. 
Rova, E. 2007. "The Late Chalcolithic period in the Tell Leilan region: A report on the ceramic material of the 1995 survey". Kaskal 4: 1-42.

2014. "Post-LC5 North Mesopotamian Developments" in M. Lebeau, ed. ARCANE Interregional 1: Ceramics. Turnhout: Brepols, pp. 1-31.

Saber, S. A., S. Salman, K. Rasheed and S. Mühl. 2014. "Two salvage excavations in Southern Kurdistan, Sulaymaniyah Province, Iraq: Tell Tanjero (season 2008) and Tell Greza (season 2003)". Mitteilungen der Deutschen Orient-Gesellschaft zu Berlin 146: 139-168.

Saed Mucheshi, A., M. Zamani Dadaneh, M. Ghasemi and Z. Karimi. 2017. "Stratigraphy at Tepe Namashir near Baneh: Western Iran". Pazhouhesh-ha-ye Bastan-shenasi-ye Iran [Archaeological Research of Iran] 7 (12): 43-62. (in Persian)

Sagona, A. G. and P. Zimansky. 2009. Ancient Turkey. London: Routledge.

Schwartz, G. M. 1988. A Ceramic Chronology from Tell Leilan, Operation 1. Yale Tell Leilan Research Vol. 1. New Haven: Yale University Press.

2001. "Syria and the Uruk Expansion" in M. S. Rothman, ed. Uruk Mesopotamia \& Its Neighbors. CrossCultural Interactions in the Era of State Formation. Santa Fe: School of American Research Press, pp. 233-264.

Sconzo, P. 2019. "Pots and places in the Late Chalcolithic period: A view from the Eastern Habur region". Paléorient 45(2): 137-162.

Skuldbøl, T.B.B. and C. Colantoni. 2016a. "Tracking early urbanism in the hilly flanks of Mesopotamia - three years of Danish archaeological investigations on the Rania Plain" in K. Kopanias and J. MacGinnis, eds. The Archaeology of the Kurdistan Region of Iraq and Adjacent Regions. Oxford: Archaeopress, pp. $411-416$.

2016b. "Early urbanism on the margins of Upper Mesopotamia - Complex settlement patterns and urban transformations on the Rania Plain in northeastern Iraq" in M. Iamoni, ed. Trajectories of Complexity: Socio-economic Dynamics in Upper Mesopotamia in the Neolithic and Chalcolithic Periods. Studia Chaburensia 6. Wiesbaden: Harrassowitz, pp. 1-26.

2018. "The Path to Urbanism. Exploring the Anatomy and Development of Early Urbanism in Northern Mesopotamia. Five Years of Investigations by the Danish Archaeological Expedition to Iraq". AshSharq Bulletin of the Ancient Near East 2/1: 1-12.

Sorkhani, R. R. and M. Eslami. 2018. "Specialized pottery production in Dalma tradition; A statistical approach in pottery analysis from Soha Chay Tepe, Zanjan, Iran”. Journal of Archaeological Science: Reports 17: 220-234.

Speiser, E. A. 1926-27. "Southern Kurdistan in the Annals of Ashurnasirpal and Today". Annual of the American Schools of Oriental Research 8: 1-41.

Starr, R.F.S. 1939. Nuzi. Cambridge: Cambridge University Press.

Stein, G. 2009. "Tell Zeidan”. Oriental Institute 2008-2009 Annual Report: 126-137.

2012. "The development of indigenous social complexity in Late Chalcolithic Upper Mesopotamia in the fifth-fourth millennia BC". Origini 34: 125-151.

_ 2018. "Excavations at Surezha, Erbil Plain, Kurdistan Region, Iraq". Oriental Institute 2017-18 Annual Report: $29-43$.

Stein, G. and A. Alizadeh. 2014. "Surezha, Kurdistan". Oriental Institute 2013-14 Annual Report: 133-146.

2015. "Preliminary Report on the First Season of Excavations at the Chalcolithic Site of Surezha in the Erbil Governorate, Kurdistan Region, Iraq, 2013". Iranian Archaeology 4: 32-41.

2017. "Excavations at Surezha (Erbil Plain, Kurdistan Region, Iraq)". Oriental Institute 2016-17 Annual Report: $73-87$.

Stein, G. and R. Özbal. 2007. "A tale of two oikumenai: variation in the expansionary dynamics between Ubaid and Uruk Mesopotamia" in E. Stone, ed. Settlement and Society. Los Angeles: Cotsen Institute of Archaeology, pp. 329-342.

Sürenhagen, D. 1979. "Ahmad al-Hattu 1978”. Mitteilungen der Deutschen Orient-Gesellschaft 111: 35-50.

Takriti, A. Q. 1960. "The Excavations at Tell ed-Daim (Dokan)”. Sumer 16: 93-110. (in Arabic).

Thornton, C. P. 2009. "The Emergence of Complex Metallurgy on the Iranian Plateau: Escaping the Levantine Paradigm". Journal of World Prehistory 22(3): 301-327.

Tobler, A. J. 1950. Excavations at Tepe Gawra II. Levels IX-XX. Philadelphia: University of Pennsylvania.

Tomé, A., R. Cabral and S. Renette. 2016. "The Kani Shaie Archaeological Project" in K. Kopanias and J. MacGinnis, eds. The Archaeology of the Kurdistan Region of Iraq and Adjacent Regions. Oxford: Archaeopress, pp. 427-434.

Tonoike, Y. 2009. Beyond Style: Petrographic Analysis of Dalma Ceramics from Two Regions in Iran. Unpublished Ph.D. dissertation, Yale University.

2012. "Petrographic Analysis of the $6^{\text {th }}$ Millennium B.C. Dalma Ceramics from Northwestern and Central Zagros”. Iranian Journal of Archaeological Studies 2: 65-82. 
Trentin, M. G. 1991. North-Western Uruk Period Pottery Assemblages. Unpublished PhD thesis, Institute of Archaeology, University College London.

Tsuneki, A., K. Rasheed, S. A. Saber, S. Nishiyama, R. Anma, B. B. Ismail, A. Hasegawa, Y. Tatsumi, Y. Miyauchi, S. Jammo, M. Makino and Y. Kudo. 2015. "Excavations at Qalat Said Ahmadan, Slemani, Iraq-Kurdistan: First Interim Report (2014 Season)". Al-Rafidan 36: 1-50.

Tsuneki, A., K. Rasheed, S. A. Saber, S. Nishiyama, N. Watanabe, T. Greenfield, B. B. Ismail, Y. Tatsumi and M. Minami. 2016. "Excavations at Qalat Said Ahmadan, Qaladizah, Iraq-Kurdistan: Second Interim Report (2015 Season)". Al-Rafidan 37: 89-142.

Tusa, S. 1984. "Excavation at Tell Abu Husaini - preliminary report". Sumer 40: 262-276.

Ur. J. 2010. Urbanism and Cultural Landscapes in Northeastern Syria: The Tell Hamoukar Survey, 1999-2001. Oriental Institute Publication 137. Chicago: Oriental Institute.

Vallet, R., J. S. Baldi, H. Naccaro, K. Rasheed, S. A. Saber and S. J. Hamarasheed. 2017. "New evidence on Uruk expansion in the Central Mesopotamian Zagros Piedmont”. Paléorient 43(1): 61-87.

Vallet, R., J. S. Baldi, M. Zingarello, M. Sauvage, H. Naccaro, C. Paladre, F. Bridey, C. Padovani, K. Rasheed, K. Raeuf and Q. Halkawt. 2019. "The emergence of cultural identities and territorial policies in the longue durée: A view from the Zagros Piedmont”. Paléorient 45(2): 163-189.

Weeks, L. 2013. "Iranian metallurgy of the fourth millennium BC in its wider technological and cultural contexts" in C. A. Petrie, ed. Ancient Iran and Its Neighbours: Local Developments and Long-Range Interactions in the Fourth Millennium BC. British Institute of Persian Studies, Archaeological Monograph Series III. Oxford: Oxbow, pp. 278-291.

Wengrow, D., R. Carter, G. Brereton, M. Shepperson, S. J. Hamarashi, S. A. Saber, A. Bevan, D. Fuller, H. Himmelman, H. Sosnowska and L. Gonzalez Carretero. 2016. "Gurga Chiya and Tepe Marani: New Excavations in the Shahrizor Plain, Iraqi Kurdistan". Iraq 77: 253-284.

Wilkinson, T. J. and D. J. Tucker. 1995. Settlement Development in the North Jazira, Iraq: A study of the archaeological landscape. Iraq Archaeological Reports 3. Warminster: British School of Archaeology in Iraq.

Wright, H. T. 2013. "A bridge between worlds: south-western Iran during the fourth millennium BC" in C. A. Petrie, ed. Ancient Iran and Its Neighbours: Local Developments and Long-Range Interactions in the Fourth Millennium BC. British Institute of Persian Studies, Archaeological Monograph Series III. Oxford: Oxbow, pp. 51-73.

Steve Renette

Department of Classical, Near Eastern, and Religious Studies

University of British Columbia

Vancouver, Canada

srenette@mail.ubc.ca

Khaled Abu Jayyab

Jackman Humanities Institute

University of Toronto

Toronto, Canada

khaled.abujayyab@mail.utoronto.ca

Elizabeth Gibbon

Department of Anthropology

University of Toronto

Toronto, Canada

elizabeth.gibbon@mail.utoronto.ca

Michael P. Lewis

Department of Archaeology

University of Cambridge

Cambridge, U.K.

m1830@cam.ac.uk 


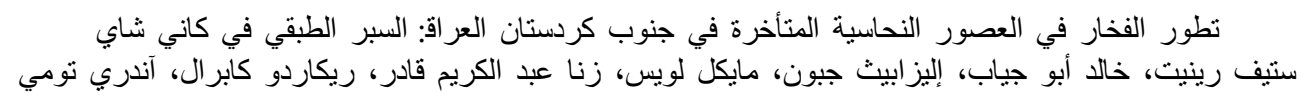

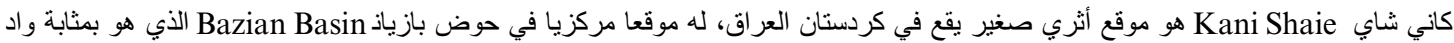

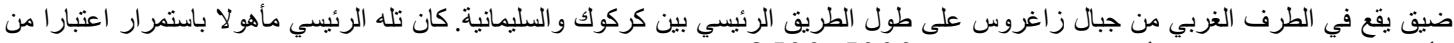

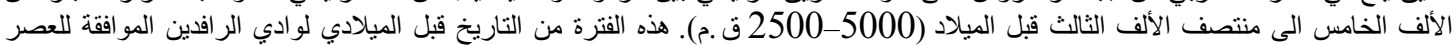

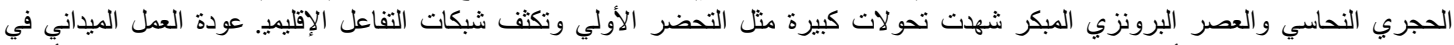

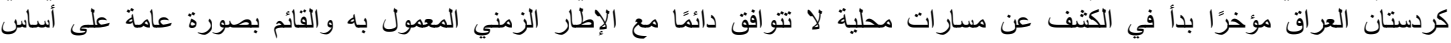

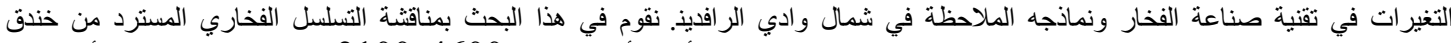

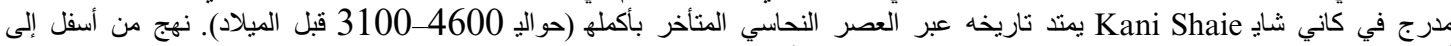

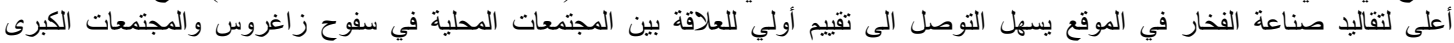

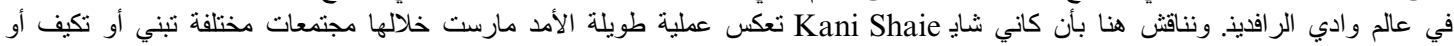

